Pontifícia Universidade $C_{\text {atólica }}$

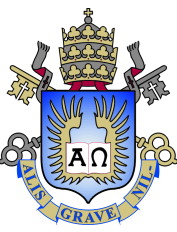

Rodrigo Marinho de Souza

Tempos de mistura para passeios aleatórios no grupo simétrico

Dissertação de Mestrado

Dissertação apresentada como requisito parcial para obtenção do grau de Mestre pelo Programa de Pós-graduação em Matemática do Departamento de Matemática do Centro Técnico Científico da PUC-Rio.

Orientador

Prof. Simon Richard Griffiths Coorientadora: Prof. Ana Patricia Carvalho Gonçalves 


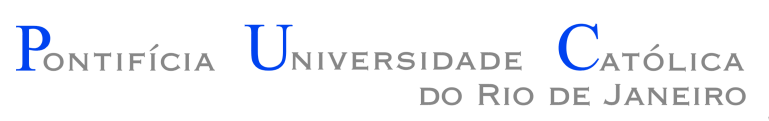

Rodrigo Marinho de Souza

\title{
Tempos de mistura para passeios aleatórios no grupo simétrico
}

\begin{abstract}
Dissertação apresentada como requisito parcial para obtenção do grau de Mestre pelo Programa de Pós-graduação em Matemática do Departamento de Matemática do Centro Técnico Científico da PUC-Rio. Aprovada pela Comissão Examinadora abaixo assinada.
\end{abstract}

Prof. Simon Richard Griffiths

Orientador Departamento de Matemática - PUC-Rio

Prof. Ana Patricia Carvalho Gonçalves

Coorientadora Instituto Técnico Superior - IST

Prof. Augusto Quadros Teixeira Instituto Nacional de Matemática Pura e Aplicada - IMPA

Prof. Freddy Rolando Hernandez Romero Universidade Federal Fluminense - UFF

Prof. Milton David Jara Valenzuela Instituto Nacional de Matemática Pura e Aplicada - IMPA

Prof. Márcio da Silveira Carvalho Coordenador Setorial do Centro Técnico Científico - PUC-Rio 
Todos os direitos reservados. É proibida a reprodução total ou parcial do trabalho sem autorização da universidade, do autor e do orientador.

Rodrigo Marinho de Souza

Terminou sua graduação em bacharelado em Matemática na Universidade Federal Fluminense, onde recebeu o Prêmio Láurea Acadêmica da UFF de 2015 pela sua dedicação como discente alcançando o maior coeficiente de rendimento dentre os formandos do ano. Foi monitor das disciplinas Pré-Cálculo e Álgebra I durante a graduação e realizou atividades de iniciação científica na área de probabilidade, estudando Cadeias de Markov e suas aplicações, como bolsista da Fundação Carlos Chagas Filho de Amparo à Pesquisa do Estado do Rio de Janeiro. Além disso, é técnico em Polímeros formado pelo Instituto Federal de Educação, Ciência e Tecnologia do Rio de Janeiro.

Ficha Catalográfica

De Souza, Rodrigo Marinho

Tempos de mistura para passeios aleatórios no grupo simétrico / Rodrigo Marinho de Souza; orientador: Simon Richard Griffiths; coorientadora: Ana Patricia Carvalho Gonçalves. - 2017.

v., 96 f: il. color. ; $30 \mathrm{~cm}$

Dissertação (mestrado) - Pontifícia Universidade Católica do Rio de Janeiro, Departamento de Matemática.

Inclui bibliografia

1. Matemática - Teses. 2. Combinatória, probabilidade e processos estocásticos - Teses. 3. Cadeias de Markov;. 4. Embaralhamentos;. 5. Tempos de Mistura;. 6. Cutoffs; 7. Processo de Exclusão Simples..

I. Griffiths, Simon Richard. II. Gonçalves, Ana Patricia Carvalho. III. Pontifícia Universidade Católica do Rio de Janeiro.

Departamento de Matemática. IV. Título. 


\section{Agradecimentos}

Primeiramente, agradeço a Deus, não apenas pela oportunidade de fazer um mestrado, mas por todas as bênçãos que derrama sobre mim, tanto na vida acadêmica quanto na vida pessoal.

Agradeço à minha família, principalmente à minha irmã Rita Marinho pelo apoio incondicional.

Aos meus amigos da PUC-Rio, pelas brincadeiras e pela bagunça, que muitas vezes serviram de escape da rotina exaustiva de estudo. Dentre estes, sinto-me obrigado a citar Edison, Simone e Thiago, cuja amizade levarei para sempre em minha vida.

Agradeço também aos professores e demais funcionários do departamento pela simpatia e dedicação. Em particular, à Creuza Nascimento e Carlos Henrique, e aos professores Carlos Tomei, cujas aulas de álgebra linear jamais esquecerei, e Marcos Craizer, pelo incentivo e constante preocupação.

Ao meu amigo professor Freddy Hernandez pelo apoio, incentivo e acompanhamento desde a graduação. Obrigado por me apresentar esta linda área de pesquisa. Devo-te muito, e espero um dia poder retribuir.

Ofereço minha eterna gratidão aos meus orientadores. Ao Simon Griffiths pela amizade, e por sempre me atender quando precisei de ajuda. À Patricia Gonçalves por dedicar grande parte de seu tempo na melhoria constante desta dissertação e pela confiança no meu trabalho.

Ao professor Hubert Lacoin, pelo maravilhoso artigo e por me ajudar a entendê-lo melhor.

Por último, porém não menos importante, agradeço à CAPES pelo apoio financeiro. 


\section{Resumo}

De Souza, Rodrigo Marinho; Griffiths, Simon Richard; Gonçalves, Ana Patricia Carvalho. Tempos de mistura para passeios aleatórios no grupo simétrico. Rio de Janeiro, 2017. 96p. Dissertação de Mestrado - Departamento de Matemática, Pontifícia Universidade Católica do Rio de Janeiro.

O objetivo desta dissertação é apresentar algumas técnicas e ferramentas para a obtenção de cotas superiores e inferiores para tempos de mistura de cadeias de Markov. Para que isso se torne mais interessante, apresentaremos estes conceitos através de cadeias de Markov que atuam sobre o grupo simétrico, que podem ser vistas como embaralhamentos de cartas. Ademais, usaremos um destes embaralhamentos como toy model para o processo de exclusão simples simétrico, o que nos ajudará a determinar os tempos de mistura do embaralhamento e do famoso sistema de partículas.

\section{Palavras-chave}

Cadeias de Markov; Embaralhamentos; Tempos de Mistura; Cutoffs; Processo de Exclusão Simples. 


\section{Abstract}

De Souza, Rodrigo Marinho; Griffiths, Simon Richard (Advisor); Gonçalves, Ana Patricia Carvalho (Co-Advisor). Mixing times for random walks on the symmetric group. Rio de Janeiro, 2017. 96p. Dissertação de Mestrado - Departamento de Matemática, Pontifícia Universidade Católica do Rio de Janeiro.

The aim of this dissertation is to introduce some techniques and tools to obtain upper and lower bounds for Markov chains mixing times. To make it more interesting, we introduce these concepts through Markov chains that act on the symmetric group, which can be seen as card shuffles. Furthermore, we use one of these shuffles as a toy model for the symmetric simple exclusion process, which helps us to determine mixing times for the shuffle and for the famous particle system.

\section{Keywords}

Markov Chains; Shuffles; Mixing Times; Cutoffs; Simple Exclusion Process. 


\section{Sumário}

1 Introdução 11

2 Tempos de mistura e distância do equilíbrio $\quad 13$

2.1 Distância de variação total 13

$\begin{array}{lll}2.2 & \text { Tempos de mistura } & 14\end{array}$

$\begin{array}{lll}2.3 & \text { Distância de separação } & 15\end{array}$

2.4 Tempos estacionários fortes 16

$\begin{array}{ll}2.5 \text { Cota por contagem } & 18\end{array}$

$\begin{array}{lll}2.6 \text { O cutoff } & 19\end{array}$

3 Embaralhamento Top-to-random 22

3.1 Introdução ao modelo 22

3.2 Cota superior 24

3.3 Cota inferior 26

$\begin{array}{lll}3.4 \text { Cutoff } & 27\end{array}$

4 O embaralhamento Gilbert-Shannon-Reeds (GSR) 29

4.1 Uma introdução ao modelo 29

4.2 Uma primeira cota superior 32

4.3 Uma primeira cota inferior 33

4.4 Generalizando o modelo: O a-embaralhamento 33

4.5 Encontrando o tempo de mistura 37

5 O embaralhamento de transposições adjacentes visto em tempo contínuo 43

5.1 O embaralhamento de transposições adjacentes 43

5.2 Tirando vantagem da monotonicidade 44

5.2.1 Mapeando permutações em superfícies discretas 45

5.2.2 A construção gráfica 46

5.2.3 Ordenação estocástica e sua preservação 48

5.2.4 Desigualdades de correlação e a desigualdade FKG 50

5.2.5 A desigualdade censuradora 52

5.2.6 Projeção e monotonia $\quad 57$

5.3 Mais algumas ferramentas $\quad 62$

5.3.1 A conexão com a equação do calor 62

5.3.2 A cota superior de Wilson 65

5.3.3 Apagando os marcadores e decompondo o procedimento de mistura 65

$\begin{array}{lll}5.4 & \text { A cota superior } & 67\end{array}$

$\begin{array}{ll}5.5 \text { A cota inferior } & 74\end{array}$

5.6 O cutoff 74

6 O embaralhamento de transposições adjacentes visto em tempo discreto $\mathbf{7 5}$

$\begin{array}{lll}6.1 \text { A cota superior } & 78\end{array}$

$\begin{array}{ll}6.2 \text { A cota inferior } & 79\end{array}$

$\begin{array}{lll}6.3 \text { O cutoff } & 79\end{array}$ 
7 Uma maravilhosa aplicação: Encontrando o tempo de mistura para o processo de exclusão simples simétrico

7.1 O processo de exclusão simples simétrico $\quad 80$

7.2 Conectando a exclusão simples e o embaralhamento de transposições adjacentes

$\begin{array}{lll}7.3 & \text { A cota superior } & 83\end{array}$

$\begin{array}{lll}7.4 & \text { A cota inferior } & 83\end{array}$

$\begin{array}{lll}7.5 \text { O cutoff } & 84\end{array}$

$\begin{array}{lr}\text { Referências Bibliográficas } & 85\end{array}$

$\begin{array}{lll}\text { A Cadeias de Markov em espaços finitos } & 87\end{array}$

$\begin{array}{lll}\text { A.1 Cadeias de Markov em tempo discreto } & 87\end{array}$

A.2 Cadeias de Markov em tempo contínuo 90

$\begin{array}{lll}\text { B Sistemas dinâmicos aleatórios } & 91\end{array}$

$\begin{array}{ll}\text { C Passeios aleatórios em Grupos } & 93\end{array}$

D Tempos de mistura analisados $\quad 96$ 


\section{Lista de figuras}

3.1 Embaralhamento top-to-random em um baralho com 7 cartas. 24

4.1 Vizualização de um embaralhamento cascata. 29

5.1 Exemplo de uma transposição adjacente. 43

7.1 Transição de uma partícula para um sítio vizinho. $\quad 81$

7.2 Permutação $e$ associada à configuração inicial das partículas na construção gráfica. $\quad 82$ 


\section{Lista de tabelas}

D.1 Tabela com todas as cadeias de Markov analisadas e seus respectivos tempos de mistura. 


\section{1 \\ Introdução}

Um passeio aleatório em um grupo é uma cadeia de Markov cujo espaço de estados é o conjunto de elementos desse grupo, e a cada passo, a probabilididade de transição de um elemento para outro é dada por um incremento que caracteriza tal passeio aleatório. Mais especificamente, esta cadeia move-se multiplicando o estado atual, à esquerda, por um elemento aleatório do grupo escolhido de acordo com o incremento.

Um embaralhamento de cartas pode ser visto como um passeio aleatório no grupo simétrico $S_{n}$ (grupo das permutações). O nosso interesse é saber quantas vezes precisamos embaralhar um baralho para que ele esteja bem embaralhado. Mas o que é estar bem embaralhado?

Um baralho tradicional possui 52 cartas, o que nos dá 52 ! permutações possíveis para ele, e isso é da ordem de $10^{68}$ que é maior que o número de partículas no sistema solar. Diremos que um baralho está bem embaralhado quando todas essas possíveis permutações forem equiprováveis, o que pode nos intimidar à saber quantas embaralhadas são necessárias e suficientes para chegarmos à uniformidade, o que chamaremos mais à frente de tempo de mistura.

Uma outra questão que podemos nos fazer é: Qual é a melhor maneira de embaralhar cartas? Esse é um problema de muito valor para cassinos e apostadores. Nesta dissertação, analisaremos três embaralhamentos. O primeiro, chamado de embaralhamento Top-to-random, consiste em pegar a carta do topo do baralho e colocá-la de volta ao baralho em qualquer posição escolhida uniformemente ao acaso. Mostraremos que o tempo de mistura para este tipo de embaralhamento é da ordem de $n \log n$, resultado obtido por Aldous e Diaconis em [1].

Em seguida, analisaremos o embaralhamento Gilbert-Shannon-Reeds (riffle shuffle), para o qual Bayer e Diaconis mostraram, em [3], que sete embaralhadas do tipo certo são necessárias e suficientes para embaralhar 
bem um baralho padrão com 52 cartas. Este resultado ficou conhecido como teorema das sete embaralhadas. Por último, porém não menos importante, estudaremos o embaralhamento das transposições adjacentes, que Lacoin em [11], usou como um toy model para o processo de exclusão simples encontrando tempos de mistura tanto para o embaralhamento, quanto para o sistema de partículas.

Ademais, mostraremos que alguns destes passeios aleatórios em $S_{n}$ apresentam um fenômeno muito interessante conhecido como cutoff, que ocorre quando a distância de variação total, que nos fornecerá a distância do equilíbrio, cai abruptamente de 1 para 0 no tempo de mistura.

É aconselhável que o leitor tenha um prévio conhecimento de álgebra linear, bem como conceitos básicos de probabilidade. E para uma leitura mais prazerosa, recomenda-se o primeiro capítulo de [12]. 


\section{2}

\section{Tempos de mistura e distância do equilíbrio}

Neste capítulo, introduziremos algumas definições e técnicas para podermos determinar o tempo que uma cadeia de Markov leva para alcançar o equilíbrio.

\section{1}

\section{Distância de variação total}

Definição 2.1 Definimos a Distância de Variação Total entre duas distribuições de probabilidade $\mu$ e $\nu$ em um espaço de estados $\Omega$ como

$$
d_{T V}(\mu, \nu)=\|\mu-\nu\|_{T V}:=\max _{A \subset \Omega}|\mu(A)-\nu(A)| .
$$

Proposição 2.2 Também podemos definir a Distância de Variação Total como

$$
\|\mu-\nu\|_{T V}=\frac{1}{2} \sum_{x \in \Omega}|\mu(x)-\nu(x)| .
$$

Prova. De fato, sejam $B=\{x: \mu(x) \geq \nu(x)\}$ e $A \subset \Omega$ um evento qualquer. Então, como $x \in\left(A \cap B^{c}\right) \Rightarrow \mu(x)<\nu(x)$, temos:

$$
\mu(A)-\nu(A) \leq \mu(A \cap B)-\nu(A \cap B) \leq \mu(B)-\nu(B)
$$

Do mesmo modo, temos:

$$
\nu(A)-\mu(A) \leq \nu\left(B^{c}\right)-\mu\left(B^{c}\right)
$$

Assim, podemos ver que os lados direitos das desigualdades acima são iguais, pois quando fazemos a diferença entre eles, obtemos zero. Daí, se $A=B$ (ou $A=B^{c}$ ), então $|\mu(A)-\nu(A)|=\nu\left(B^{c}\right)-\mu\left(B^{c}\right)$. Portanto,

$$
\begin{aligned}
\|\mu-\nu\|_{T V} & =\frac{1}{2}\left|\mu(B)-\nu(B)+\nu\left(B^{c}\right)-\mu\left(B^{c}\right)\right| . \\
& =\frac{1}{2} \sum_{x \in \Omega}|\mu(x)-\nu(x)| .
\end{aligned}
$$


Definição 2.3 Se $\nu^{(1)}, \nu^{(2)}, \ldots$ e $\nu$ são distribuições de probabilidade em $\Omega$, então dizemos que $\nu^{(t)}$ converge para $\nu$ em variação total quando $t \rightarrow \infty$, escrevendo $\nu^{(t)} \stackrel{T V}{\rightarrow} \nu$, se

$$
\lim _{t \rightarrow \infty} d_{T V}\left(\nu^{(t)}, \nu\right)=0
$$

\section{2}

\section{Tempos de mistura}

Sabemos que uma cadeia de Markov irredutível e aperiódica com espaço de estados finito $\Omega$ e matriz de transição $P$ possui uma única distribuição estacionária $\pi$ e que dada uma distribuição inicial arbitrária, essa converge para $\pi$ em variação total (veja o Teorema A.7). Estamos interessados no tempo necessário para que a cadeia atinja o equilíbrio. Dessa forma, definamos a distância entre a cadeia de Markov no tempo $t$ e a distribuição estacionária $\pi$ por

$$
d(t):=\max _{x \in \Omega}\left\|P^{t}(x, .)-\pi\right\|_{T V},
$$

onde $P^{t}(x, y)$ é a probabilidade de alcançar o estado $y$ em $t$ passos, saindo do estado $x$.

Definição 2.4 Definimos o tempo de mistura para uma cadeia de Markov por

$$
t_{m i x}(\epsilon):=\inf \{t: d(t) \leq \epsilon\}
$$

e

$$
t_{m i x}:=t_{m i x}(1 / 4)
$$

Proposição 2.5 Seja $\mu$ uma distribuição de probabilidade em $\Omega$ e $P$ uma matriz de transição. Então, $d(t)=\sup _{\mu}\left\|\mu P^{t}-\pi\right\|_{T V}$.

Prova. Seja $\mu$ uma distribuição de probabilidade em $\Omega$. Pela Proposição 2.2 e pela desigualdade triangular, temos

$$
\begin{aligned}
\left\|\mu P^{t}-\pi\right\|_{T V} & =\frac{1}{2} \sum_{y \in \Omega}\left|\mu P^{t}(y)-\pi(y)\right|=\frac{1}{2} \sum_{y \in \Omega}\left|\sum_{x \in \Omega} \mu(x) P^{t}(x, y)-\sum_{x \in \Omega} \mu(x) \pi(y)\right| \\
& \leq \frac{1}{2} \sum_{y \in \Omega} \sum_{x \in \Omega} \mu(x)\left|P^{t}(x, y)-\pi(y)\right| \\
& =\sum_{x \in \Omega} \mu(x) \frac{1}{2} \sum_{y \in \Omega}\left|P^{t}(x, y)-\pi(y)\right|=\sum_{x \in \Omega} \mu(x)\left\|P^{t}(x, .)-\pi\right\|_{T V} \\
& \leq \max _{x \in \Omega}\left\|P^{t}(x, .)-\pi\right\|_{T V}
\end{aligned}
$$


Como $\mu$ foi tomada arbitrariamente,

$$
\sup _{\mu}\left\|\mu P^{t}-\pi\right\|_{T V} \leq \max _{x \in \Omega}\left\|P^{t}(x, .)-\pi\right\|_{T V}=d(t) .
$$

A desigualdade oposta é óbvia.

\section{3}

\section{Distância de separação}

É fácil ver que a distância de variação total é uma métrica. Uma outra distância que, apesar de não ser uma métrica, é muito útil, é a distância de separação.

Definição 2.6 Definimos a distância de separação, para cada $x \in \Omega$, por

$$
s_{x}(t):=\max _{y \in \Omega}\left[1-\frac{P^{t}(x, y)}{\pi(y)}\right] \text {. }
$$

E também definimos

$$
s(t):=\max _{x \in \Omega} s_{x}(t) .
$$

Do mesmo modo, o tempo de separação pode ser definido por

$$
t_{\text {sep }}(\epsilon):=\inf \{t: s(t) \leq \epsilon\}
$$

Proposição 2.7 A distância de separação $s_{x}(t)$ satisfaz $\left\|P^{t}(x, .)-\pi\right\|_{T V} \leq$ $s_{x}(t)$, e portanto $d(t) \leq s(t)$.

Prova. A demonstração que segue encontra-se na página 80 de [12].

De fato,

$$
\begin{aligned}
\left\|P^{t}(x, .)-\pi\right\|_{T V} & =\sum_{y \in \Omega: P^{t}(x, y)<\pi(y)}\left[\pi(y)-P^{t}(x, y)\right] \\
& =\sum_{y \in \Omega: P^{t}(x, y)<\pi(y)} \pi(y)\left[1-\frac{P^{t}(x, y)}{\pi(y)}\right] \\
& \leq \max _{y \in \Omega}\left[1-\frac{P^{t}(x, y)}{\pi(y)}\right]=s_{x}(t)
\end{aligned}
$$

Nas próximas duas seções, introduziremos algumas ferramentas que utilizaremos mais à frente para encontrar cotas para os tempos de mistura. 


\section{4}

\section{Tempos estacionários fortes}

Os tempos estacionários fortes foram introduzidos pela primeira vez por Aldous e Diaconis em [2] (veja também [1]), e são uma ferramenta muito simples e útil para a obtenção de cotas superiores para tempos de mistura. As definições e resultados desta seção foram extraídos de [12].

Definição 2.8 Dada uma sequência $\left(X_{t}\right)_{t \geq 0}$, de variáveis aleatórias em $\Omega$, dizemos que uma variável aleatória $\tau$ em $\{0,1,2, \cdots, \infty\}$ é um tempo de parada para $\left(X_{t}\right)$ se, para cada $t \geq 0$, existe um conjunto $B_{t} \subset \Omega^{t+1}$ tal que

$$
\{\tau=t\}=\left\{\left(X_{0}, X_{1}, \cdots, X_{t}\right) \in B_{t}\right\}
$$

Em outras palavras, um tempo aleatório $\tau$ é um tempo de parada se, e somente se, a função indicadora $\mathbf{1}_{\{\tau=t\}}$ for uma função do vetor $\left(X_{0}, X_{1}, \cdots, X_{t}\right)$.

Toda matriz de transição $P$ tem um sistema dinâmico aleatório associado, ou seja, podemos encontrar uma sequência $\left(Z_{t}\right)_{t \geq 1}$, de variáveis aleatórias i.i.d. e uma função $f: \Omega \times \Lambda \rightarrow \Omega$ tal que a sequência $\left(X_{t}\right)_{t \geq 0}$, definida por

$$
X_{0}=x, \text { e } X_{t}=f\left(X_{t-1}, Z_{t}\right)
$$

seja uma cadeia de Markov com matriz de transição $P$ iniciada em $x$ (veja apêndice B).

Definição 2.9 Dizemos que um tempo aleatório $\tau$ é um tempo de parada randomizado para a cadeia de Markov $\left(X_{t}\right)_{t \geq 0}$ se é um tempo de parada para a sequência $\left(Z_{t}\right)_{t \geq 1}$.

Definição 2.10 Seja $\left(X_{t}\right)_{t \geq 0}$ uma cadeia de Markov irredutível com distribuição estacionária $\pi$. Um tempo estacionário $\tau$ para $\left(X_{t}\right)$ é um tempo de parada randomizado, possivelmente dependendo da posição inicial $x$, tal que a distribuição de $X_{\tau}$ é $\pi$, isto é,

$$
P_{x}\left\{X_{\tau}=y\right\}=\pi(y) .
$$

Definição 2.11 Um tempo estacionário forte para uma cadeia de Markov $\left(X_{t}\right)_{t \geq 0}$ com distribuição estacionária $\pi$ é um tempo de parada randomizado $\tau$, possivelmente dependendo da posição inicial $x$, tal que

$$
P_{x}\left\{\tau=t, X_{\tau}=y\right\}=P_{x}\{\tau=t\} \pi(y) .
$$

Em palavras, $X_{\tau}$ tem distribuição $\pi$ e é independente de $\tau$. 
Lema 2.12 Seja $\left(X_{t}\right)_{t \geq 0}$ uma cadeia de Markov irredutível com distribuição estacionária $\pi$. Se $\tau$ é um tempo estacionário forte para $\left(X_{t}\right)_{t \geq 0}$, então para todo $t \geq 0$

$$
P_{x}\left\{\tau \leq t, X_{t}=y\right\}=P_{x}\{\tau \leq t\} \pi(y)
$$

Prova. Seja $Z_{1}, Z_{2}, \cdots$ a sequência i.i.d. usada no sistema dinâmico aleatório associado a $\left(X_{t}\right)$. Para qualquer $s \leq t$,

$$
P_{x}\left\{\tau=s, X_{t}=y\right\}=\sum_{z \in \Omega} P_{x}\left\{X_{t}=y \mid \tau=s, X_{s}=z\right\} P_{x}\left\{\tau=s, X_{s}=z\right\}
$$

Como $\tau$ é um tempo de parada para $\left(Z_{t}\right)_{t \geq 0}$, o evento $\{\tau=s\}$ é equivalente a $\left\{\left(Z_{1}, \cdots, Z_{s}\right) \in B\right\}$ para algum conjunto $B \subset \Lambda^{s}$. Além disso, para inteiros $r, s \geq 0$, existe uma função $\tilde{f}_{r}: \Omega \times \Lambda^{r} \rightarrow \Omega$ tal que

$$
X_{r+s}=\tilde{f}_{r}\left(X_{s}, Z_{s+1}, \cdots, Z_{s+r}\right) .
$$

Como os vetores aleatórios $\left(Z_{1}, \cdots, Z_{s}\right)$ e $\left(Z_{s+1}, \cdots, Z_{t}\right)$ são independentes,

$$
\begin{aligned}
P_{x}\left\{X_{t}=y \mid \tau=s, X_{s}=z\right\} & =P_{x}\left\{\tilde{f}_{t-s}\left(z, Z_{s+1}, \cdots, Z_{t}\right)=y \mid\left(Z_{1}, \cdots, Z_{s}\right) \in B, X_{s}=z\right\} \\
& =P^{t-s}(z, y) .
\end{aligned}
$$

Portanto,

$$
P_{x}\left\{\tau=s, X_{t}=y\right\}=\sum_{z \in \Omega} P^{t-s}(z, y) \pi(z) P_{x}\{\tau=s\} .
$$

Como $\pi$ satisfaz $\pi=\pi P^{t-s}$,

$$
P_{x}\left\{\tau=s, X_{t}=y\right\}=\pi(y) P_{x}\{\tau=s\}
$$

Agora, somando em $s \leq t$, temos o resultado.

Proposição 2.13 Se $\tau$ é um tempo estacionário forte, então

$$
s_{x}(t) \leq P_{x}\{\tau>t\} .
$$

Prova. Sejam $x, y \in \Omega$. Então,

$$
\begin{aligned}
1-\frac{P^{t}(x, y)}{\pi(y)} & =1-\frac{P_{x}\left\{X_{t}=y\right\}}{\pi(y)} \leq 1-\frac{P_{x}\left\{X_{t}=y, \tau \leq t\right\}}{\pi(y)} \\
& =1-\frac{\pi(y) P_{x}\{\tau \leq t\}}{\pi(y)}=P_{x}\{\tau>t\}
\end{aligned}
$$


que garante o resultado.

Teorema 2.14 Se $\tau$ é um tempo estacionário forte, então

$$
d(t)=\max _{x \in \Omega}\left\|P^{t}(x, .)-\pi\right\|_{T V} \leq \max _{x \in \Omega} P_{x}\{\tau>t\}
$$

Prova. Sabemos que $d(t) \leq s(t)=\max _{x \in \Omega} s_{x}(t)$. Então, usando a Proposição 2.13, temos que

$$
d(t) \leq \max _{x \in \Omega} P_{x}\{\tau>t\}
$$

\section{5}

\section{Cota por contagem}

Definição 2.15 Seja $\left(X_{t}\right)_{t \geq 0}$ uma cadeia de Markov com matriz de transição $P$, irredutível e aperiódica em um espaço de estados $\Omega$, cuja distribuição estacionária $\pi$ é a uniforme. Definamos $d_{\text {out }}(x):=|\{y \in \Omega: P(x, y)>0\}|^{a}$ como o número de estados acessíveis em um passo saindo de $x$, e seja

$$
\Delta:=\max _{x \in \Omega} d_{\text {out }}(x)
$$

o número máximo de estados acessíveis em um passo.

Proposição 2.16 Se $\Delta^{t}<(1-\epsilon)|\Omega|$, então

$$
t_{m i x}(\epsilon) \geq \frac{\log (|\Omega|(1-\epsilon))}{\log \Delta} .
$$

Prova. De fato, seja $\Omega_{t}^{x}$ o conjunto de todos os estados acessíveis em $t$ passos saindo de $x$. Observemos que $\left|\Omega_{t}^{x}\right| \leq \Delta^{t}$.

Como $\Delta^{t}<(1-\epsilon)|\Omega|$, temos

$$
\left\|P^{t}(x, .)-\pi\right\|_{T V} \geq P^{t}\left(x, \Omega_{t}^{x}\right)-\pi\left(\Omega_{t}^{x}\right) \geq 1-\frac{\Delta^{t}}{|\Omega|}>\epsilon
$$

Então,

$$
t_{m i x}(\epsilon) \geq \frac{\log (|\Omega|(1-\epsilon))}{\log \Delta}
$$

Em particular,

$$
t_{m i x} \geq \frac{\log \left(\frac{3}{4}|\Omega|\right)}{\log \Delta}
$$




\section{6}

\section{$\mathrm{O}$ cutoff}

O cutoff é um fenômeno que ocorre em algumas cadeias de Markov quando a distância de variação total (ou de separação) cai abruptamente de 1 para 0 ao redor do tempo de mistura.

Definição 2.17 Uma sequência de cadeias de Markov tem cutoff se, para todo $0<\epsilon<1$,

$$
\lim _{n \rightarrow \infty} \frac{t_{m i x}^{(n)}(\epsilon)}{t_{m i x}^{(n)}(1-\epsilon)}=1 .
$$

Teorema 2.18 Sejam $t_{m i x}^{(n)}$ e $d_{n}$ o tempo de mistura e a distância da estacionaridade para a n-ésima cadeia numa sequência de cadeias Markov. A sequência tem cutoff se, e somente se,

$$
\lim _{n \rightarrow \infty} d_{n}\left(c t_{\text {mix }}^{(n)}\right)= \begin{cases}1, & \text { se } c<1 \\ 0, & \text { se } c>1 .\end{cases}
$$

Prova. Suponhamos que vale (2.3). Seja $0<\gamma<1$. Como $\lim _{n \rightarrow \infty} d_{n}((1-$ $\left.\gamma) t_{\text {mix }}^{(n)}\right)=1$, para cada $0<\epsilon<1$, existe $n_{1} \in \mathbb{N}$ tal que $n \geq n_{1}$ implica $\left|d_{n}\left((1-\gamma) t_{\text {mix }}^{(n)}\right)-1\right|<\epsilon$. Então, se $n \geq n_{1},-\epsilon<d_{n}\left((1-\gamma) t_{\text {mix }}^{(n)}\right)-1$, ou seja,

$$
d_{n}\left((1-\gamma) t_{\text {mix }}^{(n)}\right)>1-\epsilon
$$

Logo,

$$
(1-\gamma) t_{\text {mix }}^{(n)} \leq t_{\text {mix }}^{(n)}(1-\epsilon)
$$

Analogamente, como $\lim _{n \rightarrow \infty} d_{n}\left((1+\gamma) t_{\text {mix }}^{(n)}\right)=0$, para cada $0<\epsilon<1$, existe $n_{2} \in \mathbb{N}$ tal que $n \geq n_{2}$ implica $\left|d_{n}\left((1+\gamma) t_{\text {mix }}^{(n)}\right)\right|<\epsilon$. Logo, se $n \geq n_{2}$, temos

$$
d_{n}\left((1+\gamma) t_{\text {mix }}^{(n)}\right)<\epsilon
$$

implicando que $(1+\gamma) t_{m i x}^{(n)} \geq t_{m i x}^{(n)}(\epsilon)$. Tomemos $N=\max \left\{n_{1}, n_{2}\right\}$ e seja $n>N$. Então,

$$
\frac{t_{m i x}^{(n)}(\epsilon)}{(1+\gamma)} \leq t_{m i x}^{(n)} \leq \frac{t_{m i x}^{(n)}(1-\epsilon)}{1-\gamma} .
$$

Logo, para $0<\epsilon<\frac{1}{2}$, temos

$$
1 \leq \frac{t_{m i x}^{(n)}(\epsilon)}{t_{m i x}^{(n)}(1-\epsilon)} \leq \frac{(1+\gamma)}{1-\gamma}
$$


pois se $0<\epsilon<\frac{1}{2}$, temos que $t_{\text {mix }}^{(n)}(\epsilon) \geq t_{\text {mix }}^{(n)}(1-\epsilon)$.

Agora, fazendo $\gamma \rightarrow 0$ e $n \rightarrow \infty$, temos

$$
\lim _{n \rightarrow \infty} \frac{t_{m i x}^{(n)}(\epsilon)}{t_{m i x}^{(n)}(1-\epsilon)}=1, \text { para todo } 0<\epsilon<\frac{1}{2} .
$$

Note que se (2.4) vale, então

$$
\lim _{n \rightarrow \infty} \frac{t_{m i x}^{(n)}(1-\epsilon)}{t_{m i x}^{(n)}(\epsilon)}=1 .
$$

Logo, (2.4) também vale para todo $1>\epsilon>\frac{1}{2}$. Portanto, a sequência tem cutoff. Reciprocamente, suponhamos que a sequência tenha cutoff. Então, como

$$
\lim _{n \rightarrow \infty} \frac{t_{m i x}^{(n)}(\epsilon)}{t_{m i x}^{(n)}(1-\epsilon)}=1
$$

dado $0<\gamma<1$, existe $n_{0} \in \mathbb{N}$ tal que se $n \geq n_{0}$, então

$$
\frac{t_{m i x}^{(n)}(\epsilon)}{t_{m i x}^{(n)}(1-\epsilon)} \leq 1+\gamma=: c
$$

Assim, como $c>1, t_{m i x}^{(n)}(\epsilon) \leq c t_{m i x}^{(n)}(1-\epsilon)$. Tomando $\epsilon \in(0,1 / 2)$, temos $t_{m i x}^{(n)}(\epsilon) \leq c t_{m i x}^{(n)}(1-\epsilon) \leq c t_{m i x}^{(n)}$. Agora, pela definição de $d_{n}$,

$$
d_{n}\left(c t_{m i x}^{(n)}\right) \leq d_{n}\left(t_{m i x}^{(n)}(\epsilon)\right)=\epsilon
$$

para todo $\epsilon \in(0,1 / 2)$. Tomando $\epsilon=\frac{1}{4 n}$, para $n$ suficientemente grande, temos que $\epsilon \in(0,1 / 2)$ e

$$
\lim _{n \rightarrow \infty} d_{n}\left(c t_{m i x}^{(n)}\right) \leq \lim _{n \rightarrow \infty} \frac{1}{4 n}=0
$$

Do mesmo modo, como

$$
\lim _{n \rightarrow \infty} \frac{t_{m i x}^{(n)}(\epsilon)}{t_{m i x}^{(n)}(1-\epsilon)}=1,
$$

dado $0<\gamma<1$, existe $n_{0} \in \mathbb{N}$ tal que se $n \geq n_{0}$, então

$$
c:=(1-\gamma) \leq \frac{t_{m i x}^{(n)}(\epsilon)}{t_{m i x}^{(n)}(1-\epsilon)} .
$$

Assim, como $c<1$, $c t_{\text {mix }}^{(n)}(1-\epsilon) \leq t_{\text {mix }}^{(n)}(\epsilon)$. Tomando $\epsilon=\left(1-\frac{1}{4 n}\right)$, para $n$ suficientemente grande, temos que $c t_{m i x}^{(n)}\left(\frac{1}{4 n}\right) \leq t_{m i x}^{(n)}\left(1-\frac{1}{4 n}\right)$.

Daí,

$$
c t_{m i x}^{(n)} \leq c t_{\text {mix }}^{(n)}\left(\frac{1}{4 n}\right) \leq t_{m i x}^{(n)}\left(1-\frac{1}{4 n}\right) .
$$


Logo,

$$
d_{n}\left(c t_{m i x}^{(n)}\right) \geq\left(1-\frac{1}{4 n}\right) .
$$

Portanto,

$$
\lim _{n \rightarrow \infty} d_{n}\left(c t_{m i x}^{(n)}\right) \geq \lim _{n \rightarrow \infty}\left(1-\frac{1}{4 n}\right)=1 .
$$




\section{3}

\section{Embaralhamento Top-to-random}

\section{1}

\section{Introdução ao modelo}

Começaremos introduzindo um assunto que, a priori, não parece ter relação com embaralhamentos, mas veremos que, no final, ele nos ajudará a entender melhor o problema.

Suponha que uma empresa produz $n$ figurinhas diferentes. Um colecionador deseja um álbum completo. Suponhamos que cada figurinha que ele adquire possui probabilidade igual de ser cada um dos $n$ tipos. Assim nos perguntamos: Quantas figurinhas ele deve obter para completar o seu álbum sem trocar figurinhas com outras pessoas?

Seja $X_{t}$ ○ número de diferentes tipos entre as $t$ primeiras figurinhas do colecionador. Claramente $X_{0}=0$. Quando o colecionador tem $k$ tipos diferentes de figurinhas, tem-se $n-k$ tipos faltando. Portanto,

$$
P\left(X_{t+1}=k+1 \mid X_{t}=k\right)=\frac{n-k}{n}
$$

e

$$
P\left(X_{t+1}=k \mid X_{t}=k\right)=\frac{k}{n} .
$$

Podemos perceber que toda trajetória desta cadeia é não-decrescente e, uma vez que a cadeia chega no estado $n$ (correspondendo ao álbum completo), ela é absorvida lá.

Estamos interessados no número de passos requeridos para alcançar o estado absorvente.

Proposição 3.1 Considere um colecionador que deseja obter um álbum completo de figurinhas. Assuma que cada nova figurinha é escolhida uniforme e independentemente do conjunto de $n$ tipos possíveis, e seja $\tau$ o número (aleatório) de 
figurinhas colecionadas quando o álbum finalmente contém todos os tipos. Então

$$
\mathbb{E}(\tau)=n \sum_{k=1}^{n} \frac{1}{k} \approx n \log n .^{a}
$$

Prova. A demonstração que segue encontra-se na página 22 de [12].

A esperança $\mathbb{E}(\tau)$ pode ser calculada escrevendo $\tau$ como a soma de variáveis aleatórias geométricas. Seja $\tau_{k}$ o número total de figurinhas acumuladas quando o álbum finalmente contém $k$ figurinhas distintas. Então

$$
\tau=\tau_{n}=\tau_{1}+\left(\tau_{2}-\tau_{1}\right)+\cdots+\left(\tau_{n}-\tau_{n-1}\right)
$$

Assim, $\tau_{k}-\tau_{k-1}$ é uma variável aleatória geométrica com probabilidade de sucesso $(n-k+1) / n$, uma vez que depois de colecionar $\tau_{k-1}$ figurinhas, tem-se $n-k+1$ tipos faltando para a coleção. E cada figurinha subsequentemente obtida tem a mesma probabilidade $(n-k+1) / n$ de ser de um tipo ainda não encontrado, até que um novo tipo seja finalmente obtido. Assim, $\mathbb{E}\left(\tau_{k}-\tau_{k-1}\right)=n /(n-k+1)$ e

$$
\mathbb{E}(\tau)=\sum_{k=1}^{n} \mathbb{E}\left(\tau_{k}-\tau_{k-1}\right)=n \sum_{k=1}^{n} \frac{1}{n-k+1}=n \sum_{k=1}^{n} \frac{1}{k} \approx n \log n .
$$

Proposição 3.2 A variável aleatória /tau definida acima satisfaz $P(\tau>$ $\lceil n \log n+c n\rceil) \leq e^{-c}$ para cada $c>0$.

Prova. A demonstração que segue encontra-se na página 23 de [12].

Seja $A_{i}$ o evento que o i-ésimo tipo não aparece entre as primeiras $\lceil n \log n+c n\rceil$ figurinhas obtidas. Primeiro, observe que

$$
P(\tau>\lceil n \log n+c n\rceil)=P\left(\bigcup_{i=1}^{n} A_{i}\right) \leq \sum_{i=1}^{n} P\left(A_{i}\right) .
$$

Como cada ensaio tem probabilidade $1-n^{-1}$ de não tirar a figurinha $i$ e os ensaios são independentes, o lado direito da desigualdade (3.1) fica limitado superiormente por

$$
\sum_{i=1}^{n}\left(1-\frac{1}{n}\right)^{\lceil n \log n+c n\rceil} \leq n \exp \left(-\frac{n \log n+c n}{n}\right)=e^{-c},
$$

provando a proposição.

${ }^{a}$ Usamos $\approx$ para denotar uma aproximação assintótica. 
Agora, consideremos o seguinte método para embaralhar um baralho com $n$ cartas: Pegue a carta do topo e coloque-a aleatoria e uniformemente no baralho, como na Figura 3.1.

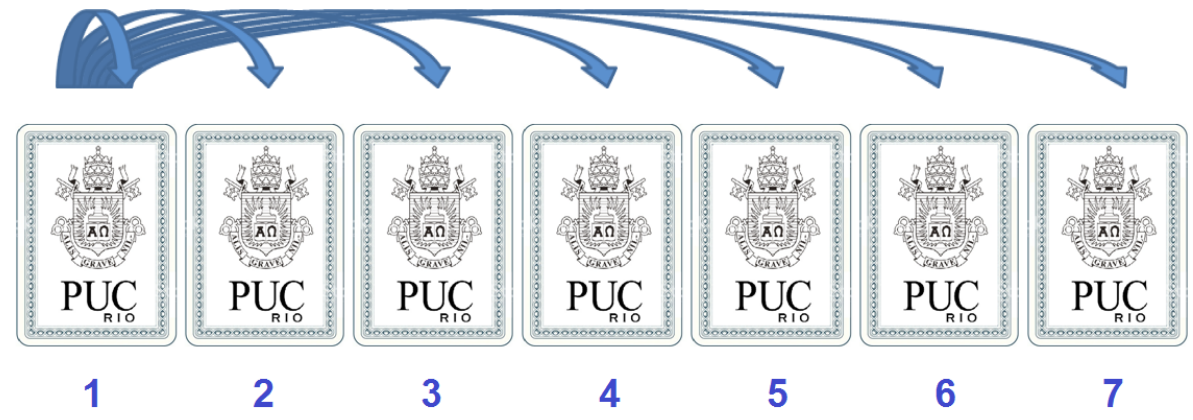

Figura 3.1: Embaralhamento top-to-random em um baralho com 7 cartas.

Esse processo, conhecido como embaralhamento top-to-random, irá misturar o baralho. As possíveis transições nas permutações do baralho são um passeio aleatório no grupo $S_{n}$, irredutível e aperiódico, que sabemos que possui uma distribuição estacionária e esta é a uniforme (veja proposições A.7 e C.2).

A pergunta agora é a seguinte: Quantas vezes devemos embaralhar este baralho usando este método para que o mesmo esteja bem embaralhado?

\section{2}

\section{Cota superior}

Definamos como $\tau_{\text {top }}$ o tempo após um movimento depois que a carta que estava originalmente no fundo do baralho é movida para o topo deste. Mostraremos que $\tau_{\text {top }}$ é um tempo estacionário forte.

Proposição 3.3 Seja $\left(X_{t}\right)_{t \geq 0}$ o passeio aleatório em $S_{n}$ correspondente ao embaralhamento top-to-random com $n$ cartas. Então, dado que em um tempo t existem $k$ cartas embaixo da que estava originalmente no fundo, cada uma das $k$ ! possíveis ordenações dessas cartas estão igualmente distribuidas. Portanto, pela definição de $\tau_{\text {top }}$, a distribuição de $X_{\tau_{\text {top }}}$ é uniforme em $S_{n}$ e o tempo $\tau_{\text {top }}$ é independente de $X_{\tau_{\text {top }}}$.

Prova. A demonstração que segue encontra-se na página 76 de [12].

Demonstraremos esta proposição por indução. Primeiro, quando $t=0$, não há cartas embaixo da carta originalmente no fundo, e portanto, a afirmação é verdadeira. 
Agora, suponhamos que a afirmação seja verdadeira para um tempo $t$. Queremos mostrar que também é para o tempo $(t+1)$.

Com efeito, existem duas possibilidades para $t+1$ : Uma carta é colocada embaixo da carta originalmente no fundo ou não. No segundo caso, as cartas embaixo da originalmente no fundo permanecem em arranjo uniforme. No primeiro caso, como uma carta foi colocada aleatoriamente embaixo da que estava originalmente no fundo, as $(k+1)$ possíveis localizações para a carta são igualmente distribuidas, e então, cada um dos $(k+1)$ ! arranjos são equiprováveis.

Além disso, em $\tau_{t o p}-1$ as cartas abaixo da inicialmente no fundo estão uniformemente distribuidas. Portanto, a ordem das cartas em $\tau_{\text {top }}$ é uniforme em todos os arranjos possíveis e $\tau_{t o p}$ é independente de $X_{\tau_{t o p}}$.

Teorema 3.4 Para o embaralhamento Top-to-Random, $t_{m i x} \leq n \log n+O(n)^{b}$.

Prova. Notemos que quando há $k$ cartas embaixo da que estava originalmente no fundo, a chance desse número aumentar em uma carta é $k / n$ até que uma embaralhada coloque a carta do topo embaixo dela. Então, a distribuição de $\tau_{t o p}$ é a mesma do tempo do colecionador de figurinhas.

Pela Proposição 3.3, vemos que $\tau_{\text {top }}$ é um tempo estacionário forte e pela Proposição 3.2, temos que para todo $c>0$,

$$
P\left(\tau_{\text {top }}>\lceil n \log n+c n\rceil\right) \leq e^{-c}
$$

Portanto, pelo Teorema 2.14, temos que

$$
d(n \log n+c n) \leq e^{-c}
$$

Consequentemente,

$$
t_{\text {mix }}(\epsilon) \leq n \log n+\log \left(\frac{1}{\epsilon}\right) n .
$$

${ }^{b}$ Dizemos que $f(n)=O(g(n))$ se $\frac{f(n)}{g(n)} \leq C$ quando $n \rightarrow \infty$. 


\section{3}

\section{Cota inferior}

Teorema 3.5 Seja $\left(X_{t}\right)$ a cadeia Top-to-Random em $n$ cartas. Para qualquer $\epsilon>0$, existe uma constante $\alpha_{0}$ tal que $\alpha>\alpha_{0}$ implica que para $n$ suficientemente grande,

$$
d_{n}(n \log n-\alpha n) \geq 1-\epsilon .
$$

Em particular, existe uma constante $\alpha_{1}$ tal que para $n$ suficientemente grande,

$$
t_{m i x} \geq n \log n-\alpha_{1} n .
$$

Prova. A demonstração que segue encontra-se na página 96 de [12].

Seja $A_{j}$ o evento em que as $j$ cartas originalmente no fundo estão em sua ordem relativa original. Seja $\tau_{j}$ o tempo necessário para a carta inicialmente na posição $j$, a contar do fundo, alcançar o topo. Então,

$$
\tau_{j}=\sum_{i=j}^{n-1} \tau_{j, i}
$$

onde $\tau_{j, i}$ é o tempo que a carta inicialmente na posição $j$, a contar do fundo, vai da posição $i$, a contar do fundo, para a posição $i+1$.

As variáveis $\tau_{j, i}, i=j, \cdots, n-1$, são independentes e $\tau_{j, i}$ tem distribuição geométrica com parametro $p=i / n$. Portanto,

$$
\mathbb{E}\left(\tau_{j, i}\right)=\frac{n}{i} \text { e } \operatorname{Var}\left(\tau_{j, i}\right)<\frac{n^{2}}{i^{2}},
$$

onde $\operatorname{Var}(X)$ é a variância da variável aleatória $X$. Assim,

$$
\mathbb{E}\left(\tau_{j}\right)=\sum_{i=j}^{n-1} \frac{n}{i}=\sum_{i=j}^{n} \frac{n}{i}-1 \geq n \log n-n \log j-n=n(\log n-\log j-1)
$$

e

$$
\operatorname{Var}\left(\tau_{j}\right) \leq n^{2} \sum_{i=j}^{\infty} \frac{1}{i(i-1)} \leq \frac{n^{2}}{j-1} .
$$

Agora, usando a desigualdade de Chebyshev, temos

$$
\begin{aligned}
P\left\{\tau_{j}<n \log n-\alpha n\right\} & \leq P\left\{\tau_{j}-\mathbb{E}\left(\tau_{j}\right)<-n(\alpha-\log j-1)\right\} \\
& \leq \frac{n^{2}}{(j-1) n^{2}(\alpha-\log j-1)^{2}} \leq \frac{1}{j-1}
\end{aligned}
$$


desde que $\alpha \geq \log j+2$.

Definamos $t_{n}(\alpha)=n \log n-\alpha n$. Se $\tau_{j} \geq t_{n}(\alpha)$, então as $j$ cartas originalmente contadas a partir do fundo do baralho estão em sua ordem relativa original em tempo $t_{n}(\alpha)$.

Logo,

$$
P^{t_{n}(\alpha)}\left(e, A_{j}\right) \geq P\left\{\tau_{j} \geq t_{n}(\alpha)\right\} \geq 1-\frac{1}{j-1},
$$

para $\alpha \geq \log j+2$.

Por outro lado, para a distribuição estacionária uniforme,

$$
\pi\left(A_{j}\right)=\frac{1}{j !} \leq \frac{1}{j-1} .
$$

Portanto, para $\alpha \geq \log j+2$,

$$
d_{n}\left(t_{n}(\alpha)\right) \geq\left\|P^{t_{n}(\alpha)}(e, .)-\pi\right\|_{T V} \geq P^{t_{n}(\alpha)}\left(e, A_{j}\right)-\pi\left(A_{j}\right)>1-\frac{2}{j-1} .
$$

Tomando $j=e^{\alpha-2}$, e $n \geq e^{\alpha-2}$, temos

$$
d_{n}\left(t_{n}(\alpha)\right)>g(\alpha):=1-\frac{2}{e^{\alpha-2}-1} .
$$

Portanto,

$$
\liminf d_{n}\left(t_{n}(\alpha)\right) \geq g(\alpha)
$$

onde $g(\alpha) \rightarrow 1$ quando $\alpha \rightarrow \infty$.

\section{4}

\section{Cutoff}

Teorema 3.6 O embaralhamento Top-to-Random tem cutoff.

Prova. De fato, para o embaralhamento Top-to-Random em um baralho com $n$ cartas, obtemos as cotas

$$
d_{n}(n \log n+\alpha n) \leq e^{-\alpha}
$$

e

$$
\liminf d_{n}(n \log n-\alpha n) \geq 1-2 e^{2-\alpha} .
$$

Em particular, a cota superior tende a 0 quando $\alpha \rightarrow \infty$, e a cota inferior tende a 1 quando $\alpha \rightarrow \infty$. Portanto, quando $n \rightarrow \infty$, a função $d_{n}$ se aproxima da função 
degrau

$$
\lim _{n \rightarrow \infty} d_{n}(c n \log n)= \begin{cases}1, & \text { se } c<1 \\ 0, & \text { se } c>1\end{cases}
$$

Portanto, pelo Teorema 2.18, o embaralhamento Top-to-Random tem cutoff. 


\section{4 \\ O embaralhamento Gilbert-Shannon-Reeds (GSR)}

4.1

\section{Uma introdução ao modelo}

Neste capítulo, vamos analizar o método mais usado, no mundo, para embaralhar cartas. $\mathrm{O}$ método baseia-se em cortar o baralho aproximadamente no meio e intercalar as cartas das duas metades como uma cascata. Por esta razão, este embaralhamento é conhecido como riffle shuffle. Veja a Figura 4.1.
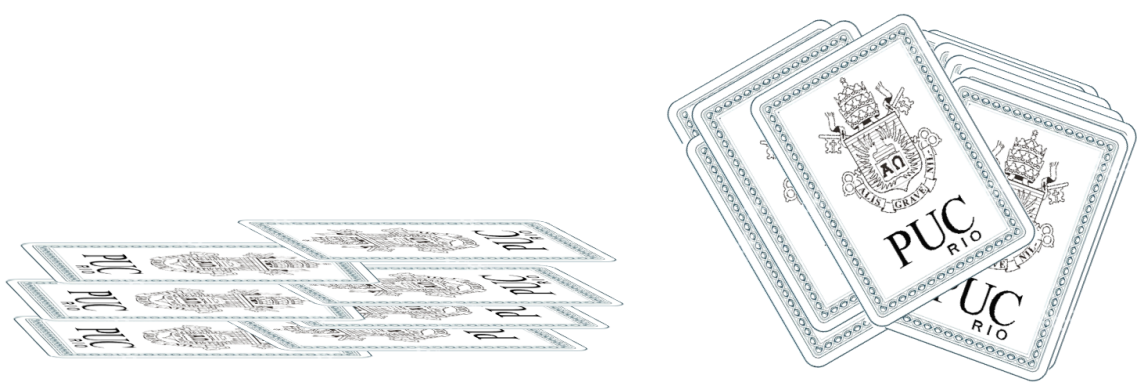

Figura 4.1: Vizualização de um embaralhamento cascata.

Definição 4.1 Uma sequência levantadora de uma permutação $\sigma$ é uma sequência maximal de valores consecutivos que ocorre na ordem relativa correta em $\sigma$.

Por exemplo, se num baralho com 10 cartas temos $e=$ $1,2,3,4,5,6,7,8,9,10$ e $\sigma=7,1,8,2,3,9,4,10,5,6$, então $\sigma$ tem duas sequências levantadoras $1,2,3,4,5,6$ e $7,8,9,10$.

O conceito de sequência levantadora foi definido através da análise de um truque de mágica chamado Premo. Não estudaremos o truque, mas este é muito bem explicado em [3].

Definição 4.2 Um riffle shuffle é uma permutação com uma ou duas sequências levantadoras, tendo apenas uma quando a permutação obtida for a identidade.

Agora analisaremos o modelo sugerido por Gilbert e Shannon (1955), e Reeds (1981), para um riffle shuffle aleatório (veja [3]). Este modelo é muito similar aos 
embaralhamentos feitos por jogadores amadores.

Abaixo listamos três métodos para embaralhar um baralho com $n$ cartas.

1. Seja $M$ uma variável aleatória binomial $(n, 1 / 2)$. Divida o baralho em duas pilhas, sendo uma delas as $M$ cartas do topo. Existem $\left(\begin{array}{c}n \\ M\end{array}\right)$ formas de juntar as duas pilhas com um riffle shuffle preservando a ordem relativa de cada pilha. Escolha um desses arranjos uniformemente ao acaso.

2. Seja $M$ uma variável aleatória binomial $(n, 1 / 2)$. Divida o baralho em duas pilhas, sendo uma delas as $M$ cartas do topo. Segure as duas pilhas sobre uma mesa e solte as cartas, uma por uma, formando uma única pilha, procedendo da seguinte maneira: Se em um momento particular a pilha da esquerda contém $a$ cartas e a pilha da direita contém $b$ cartas, então deixe cair uma carta da pilha da esquerda com probabilidade $a /(a+b)$ e uma carta da pilha da direita com probabilidade $b /(a+b)$. Repita o procedimento até que todas as cartas tenham caido.

3. Marque as $n$ cartas com $n$ marcadores "0"s ou "1"s escolhidos uniforme e independentemente, de acordo com uma moeda honesta. Uma sequência de cartas marcadas forma uma frase de "0"s e "1"s. Coloque todas as cartas marcadas com 0 no topo do baralho, preservando sua ordem relativa.

Chamaremos por $X_{t}$ a trajetória da cadeia de Markov gerada pelo embaralhamento GSR.

Lema 4.3 Os métodos 1 e 2 geram a mesma distribuição $Q$ nas permutações, onde

$$
Q(\sigma)= \begin{cases}(n+1) / 2^{n}, & \text { se } \sigma=e \\ 1 / 2^{n}, & \text { se } \sigma \text { tem exatamente duas sequências levantadoras } \\ 0, & \text { caso contrário. }\end{cases}
$$

Prova. Vejamos primeiro o método 1.

Se $\sigma$ tiver duas sequência levantadoras $1,2, \cdots, k$ e $(k+1),(k+2), \cdots, n$, então é claro, pois o evento $M=k$ acontece com probabilidade $\left(\begin{array}{l}n \\ k\end{array}\right) / 2^{n}$ e somente uma das $\left(\begin{array}{l}n \\ k\end{array}\right)$ possíveis permutações é $\sigma$. Assim, se $\mathbb{P}_{e}(\cdot)=\mathbb{P}\left(\cdot \mid X_{0}=e\right)$, temos

$$
Q(\sigma)=\mathbb{P}_{e}\left(X_{1}=\sigma, M=k\right)=\mathbb{P}_{e}\left(X_{1}=\sigma \mid M=k\right) \mathbb{P}(M=k)=\frac{1}{2^{n}}
$$


No caso de $\sigma=e$ o valor de $M$ pode ser qualquer um em entre 0 e $n$, por isso obtém-se $n+1$ vezes o valor calculado acima.

Agora analisaremos o método 2.

Dado o evento $\{M=k\}$, que tem probabilidade $\left(\begin{array}{l}n \\ k\end{array}\right) \frac{1}{2^{n}}$, mostraremos que a probabilidade de observar um certo intercalamento $\sigma$ de $1,2, \cdots, k$ e $(k+1), \cdots, n$ é igual a

$$
\frac{k !(n-k) !}{n !}=\frac{1}{\left(\begin{array}{l}
n \\
k
\end{array}\right)}
$$

Com efeito, suponhamos que $\sigma=e$. Então,

$P\left(X_{1}=\sigma \mid M=k\right)=\left(\frac{k}{n} \frac{k-1}{n-1} \cdots \frac{1}{n-k+1}\right)\left(\frac{n-k}{n-k} \frac{n-k-1}{n-k-1} \cdots \frac{1}{1}\right)=\frac{1}{\left(\begin{array}{l}n \\ k\end{array}\right)}$

Do mesmo modo, a probabilidade de gerar qualquer outra permutação $\sigma$ com sequências levantadoras $1,2, \cdots, k$ e $(k+1), \cdots, n$ é igual, pois apenas a ordem dos termos no numerador muda.

Portanto, o método 2 gera $Q$.

Proposição 4.4 Seja $\hat{Q}$ a distribuição inversa de $Q$ (veja a Definição C.7). Então o método 3 gera $\hat{Q}$, isto é, o método 3 é o embaralhamento inverso do embaralhamento GSR.

Prova. Vamos mostrar que o método 3 leva o estado $\sigma$ no estado $e$ com probabilidade $Q(\sigma)$, ou seja, $\hat{Q}\left(\sigma^{-1}\right)=Q(\sigma)$.

Com efeito, se $\sigma=e$, então $\hat{Q}(\sigma)$ é a probabilidade de todos os "0"s serem colocados antes de todos os "1"s.

Então,

$$
\hat{Q}(e)=\frac{n+1}{2^{n}}=Q(e) .
$$

Se $\sigma$ tem mais do que duas sequências levantadoras, então

$$
\hat{Q}\left(\sigma^{-1}\right)=\hat{P}(\sigma, e)=0=Q(\sigma) .
$$

Finalmente, suponhamos que $\sigma$ tenha duas sequências levantadoras, ou seja, para algum $k$, temos as sequências $1,2, \cdots, k$ e $(k+1), \cdots, n$. Para que em um passo voltemos de $\sigma$ para $e$ é necessário que a moeda dê valor 0 a todas as cartas na primeira sequência levantadora e 1 para as demais. Isso ocorre com probabilidade $\frac{1}{2^{n}}$. Portanto, $\hat{Q}\left(\sigma^{-1}\right)=Q(\sigma)$. 


\section{2}

\section{Uma primeira cota superior}

Proposição 4.5 Seja $\tau$ o número de embaralhamentos inversos necessários para que todas as cartas tenham frases (sequências de marcadores) distintas. Então, $\tau$ é um tempo estacionário forte.

Prova. Temos que provar que $\mathbb{P}\left(X_{\tau}=\sigma, \tau=t\right)=\mathbb{P}(\tau=t) \pi(\sigma)$. Com efeito, seja $t$ tal que $P(\tau=t)>0$. Então,

$$
\mathbb{P}\left(X_{\tau}=\sigma, \tau=t\right)=\mathbb{P}\left(X_{\tau}=\sigma \mid \tau=t\right) \mathbb{P}(\tau=t) .
$$

O evento $\{\tau=t\}$ garante que as $n$ cartas no tempo $t$ tenham frases distintas, mas não diz nada a respeito da ordem das cartas. Como os marcadores são escolhidos uniforme e independentemente, cada permutação das cartas tem a mesma probabilidade, que é $\frac{1}{n !}$.

Teorema 4.6 Para o embaralhamento GSR em um baralho com $n$ cartas, tem-se $t_{\text {mix }} \leq 2 \log _{2}(4 n / 3)$ para $n$ suficientemente grande.

Prova. A demonstração que segue encontra-se na página 108 de [12]. Pela Proposição C.8, Teorema 2.14 e Proposição 4.5, apenas precisamos calcular $P(\tau \leq t)$. Com efeito, se $\tau \leq t$, então no tempo $t$, todas as frases obtidas pelos marcadores são diferentes. Logo,

$$
\begin{aligned}
P(\tau \leq t) & =\frac{2^{t}}{2^{t}} \frac{2^{t}-1}{2^{t}} \frac{2^{t}-2}{2^{t}} \cdots \frac{2^{t}-(n-1)}{2^{t}} \\
& =\prod_{k=0}^{n-1}\left(1-\frac{k}{2^{t}}\right) .
\end{aligned}
$$

Então, tomando $t=2 \log _{2}(n / c)$ e usando a aproximação de $\log x$ por série de Taylor, temos:

$$
\begin{aligned}
\log \prod_{k=0}^{n-1}\left(1-\frac{k}{2^{t}}\right) & =-\sum_{k=0}^{n-1}\left(\frac{c^{2} k}{n^{2}}+O\left(\frac{k}{n^{2}}\right)^{2}\right) \\
& =-\frac{c^{2} n(n-1)}{2 n^{2}}+O\left(\frac{n^{3}}{n^{4}}\right)=-\frac{c^{2}}{2}+O\left(\frac{1}{n}\right) .
\end{aligned}
$$

Assim,

$$
\lim _{n \rightarrow \infty} \frac{P(\tau \leq t)}{e^{-c^{2} / 2}}=1
$$

Tomando $c<\sqrt{2 \log (4 / 3)} \approx 0,7585$, temos que $t_{\text {mix }} \leq 2 \log _{2}(4 n / 3)$. 


\section{3}

\section{Uma primeira cota inferior}

Teorema 4.7 Para o embaralhamento GSR em um baralho com $n$ cartas, tem-se $t_{\text {mix }} \geq \log _{2} n+O(1)$

Prova. Vamos utilizar a cota por contagem. Lembremos que $\Delta$ é o número máximo de permutações alcançaveis em um passo. Neste caso, $\Delta \leq 2^{n}$. Como $|\Omega|=n$ !, usando a cota por contagem (Proposição 2.16), temos

$$
t_{m i x} \geq \frac{\log \left(\frac{3}{4}|\Omega|\right)}{\log \Delta} \geq \frac{\log \left(\frac{3}{4} n !\right)}{n \log 2} .
$$

Agora, usando a fórmula de Stirling $\left(n ! \geq \sqrt{2 \pi} e^{-n} n^{\left(n+\frac{1}{2}\right)}\right)$, temos

$$
\begin{aligned}
t_{\text {mix }} & \geq \frac{\log \left(\frac{3}{4} \sqrt{2 \pi}\right)-n+\left(n+\frac{1}{2}\right) \log n}{n \log 2} \geq \frac{-n+n \log n}{n \log 2}=\frac{\log n}{\log 2}-\frac{1}{\log 2} \\
& =\log _{2} n+O(1) .
\end{aligned}
$$

\section{4}

\section{Generalizando o modelo: $\mathrm{O}$ a-embaralhamento}

Utilizando a primeira cota superior encontrada, temos uma estimativa de que 12 embaralhadas são suficientes para alcançarmos o equilíbrio. Para encontrarmos uma cota melhor, iremos generalizar o nosso modelo, no qual cortamos o baralho em 2 pilhas, para um modelo no qual cortamos o baralho em um número $a>1$ de pilhas.

Vejamos as seguintes descrições do novo modelo:

1. Descrição Geométrica:

- Coloquemos $n$ pontos, $x_{1}, x_{2}, \ldots, x_{n}$, de maneira uniforme e independente no intervalo $[0,1]$, de forma que $x_{1}<x_{2}<\cdots<x_{n}$.

- A aplicação $x \longmapsto a x(\bmod 1)$, que leva $[0,1]$ em $[0,1]$, preserva medida, rearranja os pontos $x_{i}$ e fornece uma medida em $S_{n}$ que chamaremos de a-embaralhamento. $(y(\bmod 1)=y-\lfloor y\rfloor)$.

2. Descrição de Entropia Máxima: Todas as maneiras de cortar o baralho em $a$ pilhas e depois intercalá-las são equiprováveis. Pilhas vazias são permitidas. 
3. Descrição Inversa: Todas as maneiras de separar um baralho embaralhado de volta em a pilhas são equiprováveis. O seguinte procedimento gera um a-embaralhamento inverso com probabilidade correta:

- Um baralho com $n$ cartas é colocado virado para baixo.

- Sucessivas cartas são viradas para cima e colocadas em uma de $a$ pilhas uniforme e independentemente.

- Depois de todas as cartas serem distribuidas, as pilhas são sobrepostas da esquerda para a direita e o baralho é virado para baixo.

4. Descrição Sequencial:

- Escolha $a$ inteiros não negativos $j_{1}, j_{2}, \cdots, j_{a}$ de acordo com a distribuição multinomial

$$
P\left(j_{1}, j_{2}, \cdots, j_{a}\right)=\frac{n !}{j_{1} ! j_{2} ! \cdots j_{a} !} \frac{1}{a^{n}} .
$$

Assim, $0 \leq j_{i} \leq n, \sum_{i=1}^{a} j_{i}=n$ e os $j_{i}$ têm a mesma distribuição que o número de bolas em cada caixa $i$ se $n$ bolas são colocadas ao acaso em $a$ caixas.

- Dados os $j_{i}$, corte as $j_{1}$ cartas do topo. Em seguida, corte as próximas $j_{2}$ cartas do topo e prossiga assim, obtendo $a$ ou menos pilhas.

- Embaralhe as duas primeiras pilhas usando o embaralhamento GSR. Depois embaralhe a pilha obtida com a terceira pilha, e prossiga assim. Isso é equivalente a intercalar todas as pilhas de uma só vez, onde se há $A_{i}$ cartas restando em uma pilha, a chance de que a próxima carta saia da pilha $i$ é $A_{i} /\left(A_{1}+\cdots+A_{a}\right)$.

Lema 4.8 As quatro descrições geram a mesma distribuição de incremento (veja a Definição C.1). Além disso, em cada modelo, um a-embaralhamento seguido de um b-embaralhamento é equivalente a um ab-embaralhamento.

Prova. Dividiremos a demonstração em três afirmações.

Afirmação 1: Cada descrição resulta em um número de cartas em cada pilha multinomial.

De fato, para a descrição sequencial isso segue pela própria definição e é claro para a descrição inversa. Para a descrição geométrica, os tamanhos das pilhas são determinados por quantos pontos são escolhidos no intervalo $[(i-1) / a,(i / a)]$, que é feito de acordo com a distribuição multinomial. Para a descrição de entropia máxima, o número de possíveis intercalamentos à partir de um corte dado é 
multinomial, pois estão em correspondência $1: 1$ com as maneiras de dividir um baralho em $a$ pilhas com seus tamanhos correspondentes.

Afirmação 2: Para as descrições de entropia máxima, inversa e sequencial, a regra do produto é válida.

De fato, dados os tamanhos das pilhas, a descrição de entropia máxima diz que todas os possíveis intercalamentos são equiprováveis. Isso também é claramente válido para a descrição inversa.

Para a descrição sequencial, observemos que quando as duas primeiras pilhas de tamanhos $j_{1}, j_{2}$ são embaralhadas, a probabilidade de uma sequência qualquer (dentre as possíveis) cair é

$$
\frac{j_{1}\left(j_{1}-1\right) \cdots 1 j_{2}\left(j_{2}-1\right) \cdots 1}{\left(j_{1}+j_{2}\right)\left(j_{1}+j_{2}-1\right) \cdots 1}=\frac{1}{\left(\begin{array}{c}
j_{1}+j_{2} \\
j_{2}
\end{array}\right)}
$$

Quando esta pilha resultante é embaralhada com a terceira pilha (de tamanho $j_{3}$ ), todas as $\left(\begin{array}{c}j_{1}+j_{2}+j_{3} \\ j_{3}\end{array}\right)$ possíveis sequências de cartas são equiprováveis. Prosseguindo indutivamente, todos os possíveis intercalamentos são equiprováveis.

Como vimos, nenhuma informação sobre os estados é retida entre embaralhamentos nestes três modelos, então a regra do produto para uma sequência de embaralhamentos vale em cada modelo uma vez que está estabelecida para um deles.

Assim, basta olharmos para a descrição inversa:

Combinando lexicograficamente os marcadores das pilhas de um a-embaralhamento inverso com os de um b-embaralhamento inverso, temos marcadores das pilhas de um ab-embaralhamento inverso. Para ver isso, seja $A_{i}$ o evento em que o marcador $a_{i}$ é escolhido para uma carta em um a-embaralhamento inverso e $B_{j}$ o evento em que o marcador $b_{j}$ é escolhido para a mesma carta em um b-embaralhamento inverso. Então,

$$
P\left(A_{i}, B_{j}\right)=P\left(A_{i}\right) P\left(B_{j}\right)=\frac{1}{a} \frac{1}{b}=\frac{1}{a b}=P\left((A B)_{k}\right),
$$

onde $(A B)_{k}$ é o evento em que o marcador $(a b)_{k}$ é escolhido para aquela carta em um ab-embaralhamento inverso.

Afirmação 3: Para a descrição geométrica, a regra do produto segue da independência de dígitos na base $a$ dos pontos escolhidos uniformemente em $[0,1]$. De fato, escolher $n$ pontos em $[0,1]$, marcá-los com os seus dígitos líderes (na base a) e aplicar $x \longmapsto a x(\bmod 1)$, é o mesmo que escolher $n$ pontos em $[0,1] \mathrm{e}$ marcá-los com inteiros $\{0,1, \cdots, a-1\}$ escolhidos uniformemente. Assim, como a 
descrição geométrica nos permite usar os pontos para embaralhamentos sucessivos, o resultado segue da identidade $b(\operatorname{ax}(\bmod 1))(\bmod 1)=a b x(\bmod 1)$.

Teorema 4.9 Seja E o evento em que um a-embaralhamento resulta em uma permutação $\sigma$ com $r$ sequências levantadoras. Então,

$$
P(E)=\frac{\left(\begin{array}{c}
a+n-r \\
n
\end{array}\right)}{a^{n}} .
$$

Prova. Usando a descrição de entropia máxima, essa probabilidade é determinada pelo número de maneiras de cortar um baralho ordenado em $a$ pilhas de maneira que $\sigma$ seja um intercalamento possível. Como cada pacote mantém sua ordem original quando as cartas são intercaladas juntas, cada sequência levantadora no baralho é uma união de pilhas. Assim, queremos contar o número de maneiras de termos $r$ sequências levantadoras em $a$ pilhas. Faremos essa conta usando o argumento de estrelas e barras usado em combinatória. Devemos colocar $(a-1)$ barras (cortes) entre as cartas, gerando $a$ pilhas, mas $(r-1)$ dessas barras devem estar entre os pares sucessivos de sequências levantadoras. As $(a-r)$ barras restantes podem estar em qualquer posição. Agora, consideremos $(a+n-r)$ objetos, sendo $n$ cartas e $(a-r)$ barras. Então, o número de maneiras de colocarmos as barras entre as cartas é $\left(\begin{array}{c}a+n-r \\ n\end{array}\right)$, e este é o número de maneiras de dividirmos o baralho com $n$ cartas em $a$ pilhas de modo que $\sigma$ seja uma configuração possível. Além disso, o número total de cortes possíveis para a obtenção de $a$ pilhas é $a^{n}$, pois podemos associar cada carta a um dos a pacotes. Portanto,

$$
P(E)=\frac{\left(\begin{array}{c}
a+n-r \\
n
\end{array}\right)}{a^{n}} .
$$

O resultado a seguir não será utilizado em nossos problemas, mas é uma identidade famosa que pode ser provada facilmente usando o que temos até agora.

Corolário 4.10 (Identidade de Worpitzky's) Sejam $A_{n, r}$ os números de Euler. Então,

$$
a^{n}=\sum_{r=1}^{n} A_{n, r}\left(\begin{array}{c}
a+n-r \\
n
\end{array}\right) .
$$


Prova. Existem $A_{n, r}$ permutações com $r$ sequências levantadoras. Assim, se $R$ é o conjunto de todas as permutações com $r$ sequências levantadoras, então

$$
\sum_{r=1}^{n} \sum_{\sigma \in R} \frac{\left(\begin{array}{c}
a+n-r \\
n
\end{array}\right)}{a^{n}}=1
$$

Logo,

$$
a^{n}=\sum_{r=1}^{n} A_{n, r}\left(\begin{array}{c}
a+n-r \\
n
\end{array}\right) .
$$

Corolário 4.11 Se um baralho recebe uma sequência de $m$ embaralhamentos dos tipos $a_{1}, a_{2}, \cdots, a_{m}$, então a probabilidade de que o baralho esteja no arranjo $\sigma$ é dada por

$$
\frac{\left(\begin{array}{c}
a+n-r \\
n
\end{array}\right)}{a^{n}},
$$

onde $a=a_{1} a_{2} \cdots a_{m}$ e $r$ é o número de sequências levantadoras em $\sigma$.

Prova. Basta combinarmos o Lema 4.8 com o Teorema 4.9.

Teorema 4.12 Se $n$ cartas são embaralhadas $m$ vezes com o embaralhamento GSR, então a probabilidade de que o baralho esteja no arranjo $\sigma$ é dada por $\frac{\left(\begin{array}{c}2^{m}+n-r \\ n\end{array}\right)}{2^{m n}}$, onde $r$ é o número de sequências levantadoras em $\sigma$.

Prova. Segue imediatamente do Corolário 4.11.

\section{5}

\section{Encontrando o tempo de mistura}

Proposição 4.13 Seja $Q^{m}(r)=\left(\begin{array}{c}2^{m}+n-r \\ n\end{array}\right) / 2^{m n}$ a probabilidade de termos uma permutação com $r$ sequências levantadoras depois de $m$ embaralhadas com distribuição GSR. Seja $r=n / 2+h$, onde $-n / 2+1 \leq h \leq n / 2$ e tomemos $m=\log _{2}\left(n^{3 / 2} c\right)$ para algum $c>0$. Então,

$$
Q^{m}(r)=\frac{1}{n !} \exp \left\{\frac{1}{c \sqrt{n}}\left(-h+\frac{1}{2}+O_{c}\left(\frac{h}{n}\right)\right)-\frac{1}{24 c^{2}}-\frac{1}{2}\left(\frac{h}{c n}\right)^{2}+O_{c}\left(\frac{1}{n}\right)\right\} .
$$


Prova.

$$
\begin{aligned}
Q^{m}(r) & =\frac{\left(2^{m}+n-r\right) !}{n !\left(2^{m}-r\right) ! 2^{m n}} \\
& =\frac{1}{n !}\left[\frac{\left(2^{m}+n-r\right) \cdots\left(2^{m}+1-r\right)\left(2^{m}-r\right) !}{\left(2^{m}-r\right) ! 2^{m n}}\right] \\
& =\frac{1}{n !}\left(\frac{2^{m}+n-r}{2^{m}} \cdots \frac{2^{m}+1-r}{2^{m}}\right) \\
& =\frac{1}{n !} \exp \left\{\sum_{i=0}^{n-1} \log \left(1+\frac{(n-i)-r}{2^{m}}\right)\right\} \\
& =\frac{1}{n !} \exp \left\{\sum_{i=0}^{n-1} \log \left(1+\frac{(n-i)-r}{c n^{3 / 2}}\right)\right\} \\
& =\frac{1}{n !} \exp \left\{\sum_{i=0}^{n-1} \log \left(1+\frac{(n-i)-n / 2-h}{c n^{3 / 2}}\right)\right\} \\
& =\frac{1}{n !} \exp \left\{\sum_{i=0}^{n-1} \log \left(1+\frac{n / 2-h-i}{c n^{3 / 2}}\right)\right\} .
\end{aligned}
$$

Afirmação: $-\frac{1}{2}<\frac{n / 2-h-i}{c n^{3 / 2}}<1$, para $n$ suficientemente grande.

De fato, para $0 \leq i \leq n-1$, temos

$$
n / 2-h-i \leq \frac{n}{2}+\left(\frac{n}{2}-1\right)-i=n-1-i \leq n-1 \leq n .
$$

Daí,

$$
\frac{n / 2-h-i}{c n^{3 / 2}} \leq \frac{n}{c n^{3 / 2}}=\left(\frac{1}{c}\right)\left(\frac{1}{\sqrt{n}}\right) .
$$

Por outro lado,

$$
n / 2-h-i \geq \frac{n}{2}-\frac{n}{2}-i=-i \geq-n
$$

Daí,

$$
\frac{n / 2-h-i}{c n^{3 / 2}} \geq-\frac{n}{c n^{3 / 2}}=\left(-\frac{1}{c}\right)\left(\frac{1}{\sqrt{n}}\right) .
$$

Logo, para $n$ suficientemente grande, $-\frac{1}{2}<\frac{n / 2-h-i}{c n^{3 / 2}}<1$, para todo $i \in$ $\{0, \ldots, n-1\}$.

Veja também que para $n$ suficientemente grande

$$
\begin{gathered}
\frac{1}{c n^{3 / 2}} \sum_{i=0}^{n-1}(n / 2-h-i)=\frac{-h+1 / 2}{c \sqrt{n}}, \\
\frac{1}{2 c^{2} n^{3}} \sum_{i=0}^{n-1}(n / 2-h-i)^{2}=\frac{1}{24 c^{2}}+\frac{1}{2}\left(\frac{h}{c n}\right)^{2}+O_{c}\left(\frac{1}{n}\right), \\
\frac{1}{3 c^{3} n^{9 / 2}} \sum_{i=0}^{n-1}(n / 2-h-i)^{3}=O_{c}\left(\frac{h}{n^{3 / 2}}\right),
\end{gathered}
$$




$$
\frac{1}{c^{4} n^{6}} \sum_{i=0}^{n-1}(n / 2-h-i)^{4}=O_{c}\left(\frac{1}{n}\right)
$$

Agora, usando o fato de que

$$
x-\frac{x^{2}}{2}+\frac{x^{3}}{3}-x^{4} \leq \log (1+x) \leq x-\frac{x^{2}}{2}+\frac{x^{3}}{3},
$$

para $-\frac{1}{2}<x<1$, e usando (4.2), (4.3), (4.4) e (4.5) em (4.1), temos que

$$
\begin{aligned}
Q^{m}(r) & =\frac{1}{n !} \exp \left\{\frac{-h+1 / 2}{c \sqrt{n}}-\left(\frac{1}{24 c^{2}}+\frac{1}{2}\left(\frac{h}{c n}\right)^{2}+O_{c}\left(\frac{1}{n}\right)\right)+O_{c}\left(\frac{h}{n^{3 / 2}}\right)+O_{c}\left(\frac{1}{n}\right)\right\} \\
& =\frac{1}{n !} \exp \left\{\frac{1}{c \sqrt{n}}\left(-h+\frac{1}{2}+O_{c}\left(\frac{h}{n}\right)\right)-\frac{1}{24 c^{2}}-\frac{1}{2}\left(\frac{h}{c n}\right)^{2}+O_{c}\left(\frac{1}{n}\right)\right\}
\end{aligned}
$$

Note que a probabilidade $Q^{m}(r)$ é monótona decrescente em $r$ para $m$ fixo. A próxima proposição determinará quando ela alcança o ponto $1 / n$ !.

Proposição 4.14 Com a notação da Proposição 4.13, seja $h^{*}$ um inteiro tal que $Q^{m}(n / 2+h) \geq 1 / n ! \Leftrightarrow h \leq h^{*}$. Então, para qualquer $c$ fixo, quando $n \rightarrow \infty$,

$$
h^{*}=\frac{-\sqrt{n}}{24 c}+\frac{1}{12 c^{3}}+B+O_{c}\left(\frac{1}{\sqrt{n}}\right)
$$

onde $-1 \leq B \leq 1$

Prova. Pela Proposição 4.13, $Q^{m}(n / 2+h) \geq 1 / n$ ! se, e somente se,

$$
\frac{1}{c \sqrt{n}}\left(-h+\frac{1}{2}+O_{c}\left(\frac{h}{n}\right)\right)-\frac{1}{24 c^{2}}-\frac{1}{2}\left(\frac{h}{c n}\right)^{2}+O_{c}\left(\frac{1}{n}\right) \geq 0 .
$$

Assim, basta tomarmos $h^{*}$ tal que

$$
\frac{1}{c \sqrt{n}}\left(-h^{*}+\frac{1}{2}+O_{c}\left(\frac{h^{*}}{n}\right)\right)-\frac{1}{24 c^{2}}-\frac{1}{2}\left(\frac{h^{*}}{c n}\right)^{2}+O_{c}\left(\frac{1}{n}\right)=0 .
$$

O próximo resultado é o principal teorema deste capítulo, que combina as estimativas acima para obtermos o comportamento assintótico da variação total. Para isso, precisaremos da Proposição 2 de [16], que diz que se $b_{n}=x \sqrt{n / 12}+n / 2$, então

e

$$
\lim _{n \rightarrow \infty} \sqrt{\frac{n}{12}} \frac{1}{n !} A_{n,\left\lfloor b_{n}\right\rfloor}=\frac{1}{\sqrt{2 \pi}} e^{-\left(x^{2} / 2\right)}
$$

$$
\lim _{n \rightarrow \infty} \frac{1}{n !} \sum_{h=1}^{\left\lfloor b_{n}\right\rfloor} A_{n, h}=\Phi(x),
$$

onde $\Phi(x)=\int_{-\infty}^{x} e^{-t^{2} / 2} d t / \sqrt{2 \pi}$. 
Teorema 4.15 Sejam $Q^{m}$ a distribuição do embaralhamento GSR no grupo simétrico $S_{n}$ e $U$ a distribuição uniforme. Se $m=\log _{2}\left(n^{3 / 2} c\right)$, com $0<c<\infty$ fixo, então, quando $n \rightarrow \infty$,

$$
d(m)=\left\|Q^{m}-U\right\|_{T V}=1-2 \Phi\left(\frac{-1}{4 c \sqrt{3}}\right)+O_{c}\left(\frac{1}{n^{1 / 4}}\right) .
$$

Prova. Seja $R_{n, h}$ o número de permutações com $n / 2+h$ sequências levantadoras. Pela Proposição 4.14, temos que

$$
d(m)=\sum_{\sigma \in S_{n}}\left(Q^{m}(\sigma)-\frac{1}{n !}\right)^{+}=\sum_{-n / 2<h \leq h^{*}} R_{n, h}\left(Q^{m}\left(\frac{n}{2}+h\right)-\frac{1}{n !}\right) .
$$

Tomando $x(n):=\frac{h}{\sqrt{n / 12}}=: x_{n}$ e substituindo em (4.6), obtemos

$$
\frac{R_{n, h}}{n !}=\frac{A_{n, n / 2+h}}{n !}=\frac{e^{-(1 / 2) x_{n}{ }^{2}}}{\sqrt{2 \pi n / 12}}\left(1+o\left(\frac{1}{\sqrt{n}}\right)\right) .
$$

Já substituindo em (4.7), obtemos

$$
\begin{aligned}
\frac{1}{n !} \sum_{h=-n / 2+1}^{h^{*}} R_{n, h} & =\frac{1}{n !} \sum_{h=-n / 2+1}^{h^{*}} A_{n, n / 2+h} \\
& =\frac{1}{n !} \sum_{h=1}^{h^{*}+n / 2} A_{n, h} \\
& =\Phi\left(\frac{h^{*}}{\sqrt{n / 12}}\right)=\Phi\left(\frac{2 \sqrt{3} h^{*}}{\sqrt{n}}\right) \\
& =\Phi\left(\frac{2 \sqrt{3}\left[-\frac{\sqrt{n}}{24 c}+O_{c}(1)\right]}{\sqrt{n}}\right) \\
& =\Phi\left(\frac{-\sqrt{3}}{12 c}+O_{c}\left(\frac{1}{\sqrt{n}}\right)\right) \\
& =\Phi\left(\frac{-1}{4 c \sqrt{3}}+O_{c}\left(\frac{1}{\sqrt{n}}\right)\right) \\
& =\Phi\left(\frac{-1}{4 c \sqrt{3}}\right)\left(1+O_{c}\left(\frac{1}{\sqrt{n}}\right)\right) .
\end{aligned}
$$

A soma em (4.8) pode ser quebrada em duas zonas:

$$
\text { zona } 1:\left\{\frac{-10 n^{3 / 4}}{\sqrt{c}} \leq h \leq h^{*}\right\}=I_{1} \text {, }
$$

e

$$
\text { zona 2: }\left\{-\frac{n}{2}<h<\frac{-10 n^{3 / 4}}{\sqrt{c}}\right\}=I_{2} \text {. }
$$


Será mostrado que apenas a zona 1 contribui. Antes de realizarmos as contas, vejamos que em $I_{1}$

$$
x_{n}=\frac{h}{\sqrt{n / 12}} \geq \frac{-10 \sqrt{12} n^{3 / 4}}{n^{1 / 2} \sqrt{c}}=-20 \sqrt{3} n^{1 / 4} / \sqrt{c}
$$

e

$x_{n}=\frac{h}{\sqrt{n / 12}} \leq \frac{h^{*}}{\sqrt{n / 12}}=\frac{\left(\frac{-\sqrt{n}}{24 c}+O_{c}(1)\right) \sqrt{12}}{\sqrt{n}}=\frac{-\sqrt{12}}{24 c}+o(1)=-\frac{1}{2 c \sqrt{12}}+o(1)$

Por (4.9) e pela Proposição 4.13,

$$
\begin{aligned}
& \sum_{I_{1}} R_{n, h} Q^{m}(n / 2+h) \\
& =\frac{e^{\frac{-1}{24 c^{2}}}}{\sqrt{2 \pi n / 12}} \sum_{I_{1}} \exp \left\{-\frac{1}{2}\left(\frac{h}{\sqrt{n / 12}}\right)^{2}-\frac{h}{c \sqrt{n}}+O_{c}\left(\frac{1}{n^{1 / 4}}\right)\right\}\left\{1+o\left(\frac{1}{\sqrt{n}}\right)\right\} \\
& =\frac{e^{\frac{-1}{24 c^{2}}}}{\sqrt{2 \pi}}\left(\frac{1}{\sqrt{n / 12}} \sum_{I_{1}} \exp \left\{-\frac{x_{n}^{2}}{2}-\frac{x_{n}}{2 c \sqrt{3}}+O_{c}\left(\frac{1}{n^{1 / 4}}\right)\right\}\right)\left\{1+o\left(\frac{1}{\sqrt{n}}\right)\right\} \\
& =\frac{e^{\frac{-1}{24 c^{2}}}}{\sqrt{2 \pi}}\left(\frac{1}{\sqrt{n / 12}} \sum_{x=\left\lceil-20 \sqrt{3} n^{1 / 4} / \sqrt{c}\right\rceil}^{\left\lfloor-(2 c \sqrt{12})^{-1}+o(1)\right\rfloor} \exp \left\{-\frac{x^{2}}{2}-\frac{x}{2 c \sqrt{3}}+O_{c}\left(\frac{1}{n^{1 / 4}}\right)\right\}\right) \times \\
& \times\left\{1+o\left(\frac{1}{\sqrt{n}}\right)\right\} \\
& =\frac{e^{\frac{-1}{24 c^{2}}}}{\sqrt{2 \pi}} \int_{-\infty}^{-(2 c \sqrt{12})^{-1}} e^{-x^{2} / 2-x /(2 c \sqrt{3})} d x\left(1+O_{c}\left(\frac{1}{n^{1 / 4}}\right)\right) \\
& =\frac{1}{\sqrt{2 \pi}} \int_{-\infty}^{-(2 c \sqrt{12})^{-1}} e^{-x^{2} / 2-x /(2 c \sqrt{3})-1 /\left(24 c^{2}\right)} d x\left(1+O_{c}\left(\frac{1}{n^{1 / 4}}\right)\right) \\
& =\frac{1}{\sqrt{2 \pi}} \int_{-\infty}^{-(2 c \sqrt{12})^{-1}} e^{-\frac{(x+1 /(2 c \sqrt{3}))^{2}}{2}} d x\left(1+O_{c}\left(\frac{1}{n^{1 / 4}}\right)\right) \\
& =\frac{1}{\sqrt{2 \pi}} \int_{-\infty}^{(4 c \sqrt{3})^{-1}} e^{-\frac{y^{2}}{2}} d y\left(1+O_{c}\left(\frac{1}{n^{1 / 4}}\right)\right) \\
& =\Phi\left(\frac{1}{4 c \sqrt{3}}\right)\left(1+O_{c}\left(\frac{1}{n^{1 / 4}}\right)\right) \\
& =\left[1-\Phi\left(\frac{-1}{4 c \sqrt{3}}\right)\right]\left(1+O_{c}\left(\frac{1}{n^{1 / 4}}\right)\right) \text {. }
\end{aligned}
$$

Na zona $2, Q^{m}(n / 2+h) \leq Q^{m}(1) \leq e^{\sqrt{n} /(2 c)} / n$ !. A cota de grandes desvios padrões, como no capítulo 16 de [7] aplicada à soma de $n$ variáveis aleatórias 
uniformes garante que

$$
\begin{aligned}
\sum_{I_{2}} R_{n, h} Q^{m}(n / 2+h) & \leq \sum_{I_{2}} \frac{R_{n, h} e^{\sqrt{n} /(2 c)}}{n !} \\
& =e^{\sqrt{n} /(2 c)} \sum_{I_{2}} \frac{R_{n, h}}{n !} \\
& \sim \frac{e^{\sqrt{n} /(2 c)}}{10 n^{1 / 4} \sqrt{2 \pi}} \exp \left[-\frac{1}{2}\left(\frac{10 \sqrt{12} n^{1 / 4}}{\sqrt{c}}\right)^{2}\right],
\end{aligned}
$$

que converge para 0 quando $n \rightarrow \infty$.

O resultado segue por (4.8), (4.10), (4.11) e (4.12).

Corolário 4.16 Se $n$ cartas são embaralhadas $m$ vezes com $m=\frac{3}{2} \log _{2} n+\theta$, então para $n$ suficientemente grande,

$$
d(m)=\left\|Q^{m}-U\right\|_{T V}=1-2 \Phi\left(\frac{-2^{-\theta}}{4 \sqrt{3}}\right)+O\left(\frac{1}{n^{1 / 4}}\right) .
$$

Prova. Basta tomarmos $c=2^{\theta}$ e o resultado segue imediatamente do Teorema 4.15.

Embora a distância de variação total fique menor que $1 / 4$ apenas depois de oito embaralhadas, este teorema é conhecido como teorema das sete embaralhadas pois é a partir deste número que a distância de variação total fica menor que $1 / 2$. 


\section{5 \\ O embaralhamento de transposições adjacentes visto em tempo contínuo}

Até agora, sempre analisamos a mistura dos passeios aleatórios em $S_{N}$ em tempo discreto. Também faremos isso para o embaralhamento de transposições adjacentes, porém no Capítulo 6. Antes, analisaremos este embaralhamento quando o mesmo é visto em tempo contínuo, o que nos ajudará não apenas a resolver o problema em tempo discreto, mas também nos dará uma bela aplicação em sistemas de partículas que será apresentada no Capítulo 7.

\section{1}

\section{O embaralhamento de transposições adjacentes}

O embaralhamento das transposições adjacentes é uma cadeia de Markov em tempo contínuo em $S_{N}$. Suponhamos que tenhamos um baralho com $N$ cartas marcadas de 1 até $N$ do topo para o fundo. Assim, para um vetor de cartas, associamos uma permutação $\sigma$ dizendo que $\sigma(x)=y$ se a $x$-ésima posição na pilha está ocupada pela carta $y$. Nossa cadeia escolhe uma carta uniformemente ao acaso dentre as cartas nas posições 1 até $N-1$ e troca sua posição com a da carta que está imediatamente abaixo desta. Veja um exemplo na Figura 5.1

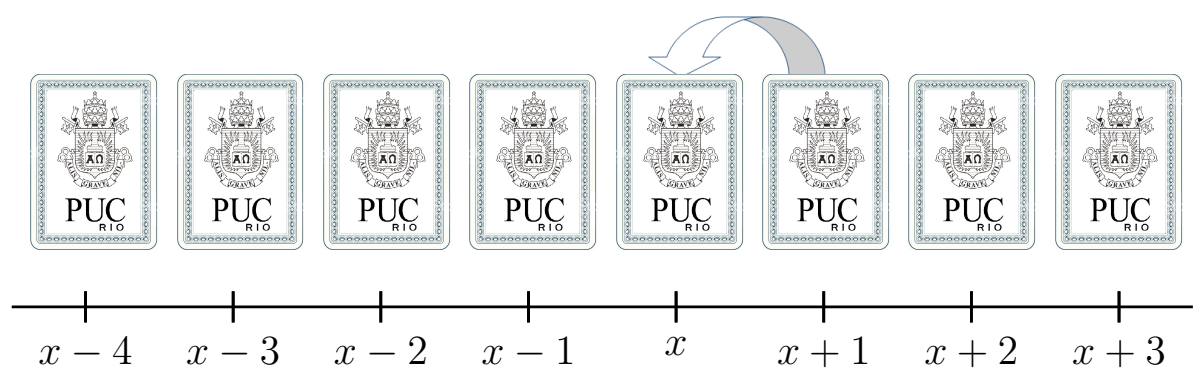

Figura 5.1: Exemplo de uma transposição adjacente.

Mais formalmente, sejam $\left(\tau_{x}\right)_{1 \leq x \leq N-1}$ as transposições para o vizinho mais próximo $(x, x+1)$. 
Capítulo 5. O embaralhamento de transposições adjacentes visto em tempo contínuo

Note que o conjunto $\left\{\tau_{x}: 1 \leq x \leq N-1\right\}$ é um gerador de $S_{N}$ no sentido da teoria de grupos.

O gerador $\mathcal{L}$ (veja $[13,14])$ do embaralhamento é definido por sua ação nas funções de $\mathbb{R}^{\Omega}$ como:

$$
(\mathcal{L} f)(\sigma):=\sum_{x=1}^{N-1} f\left(\sigma \circ \tau_{x}\right)-f(\sigma) .
$$

Sejam $\left(\sigma_{t}\right)_{t \geq 0}$ a trajetória da cadeia de Markov com $\sigma_{0}=e$ (a identidade) e $P_{t}$ a lei de distribuição na marginal $\sigma_{t}$. Dada uma distribuição de probabilidade $\nu$, definimos $P_{t}^{\nu}$ como a distribuição marginal de $\sigma_{t}^{\nu}$, a cadeia de Markov começando com distribuição inicial $\nu$. Além disso, como $\left(\tau_{x}\right)_{x=1}^{N-1}$ gera o grupo $S_{N}$, essa cadeia de Markov é irredutível e a única medida de probabilidade invariante é a uniforme $\pi$.

Agora, definamos a distância do equilíbrio dessa cadeia de Markov como

$$
d^{N}(t):=\left\|P_{t}-\pi\right\|_{T V}
$$

Pela simetria de $S_{N}, d^{N}$ não depende da condição inicial e pela Proposição 2.5 ,

$$
d^{N}(t):=\max _{\left\{\nu \text { probabilidade em } S_{N}\right\}}\left\|P_{t}^{\nu}-\pi\right\|_{T V}
$$

Finalmente, para $\epsilon \in(0,1)$, definiremos o $\epsilon$-tempo de mistura como

$$
T_{m i x}^{N}(\epsilon):=\inf \left\{t \geq 0: d^{N}(t) \leq \epsilon\right\} .
$$

Algumas vezes, para que alguns conceitos fiquem mais claros, tentaremos explicá-los usando um baralho usual com 52 cartas. Por convenção, nossa identidade estará organizada de modo que as cartas vermelhas estejam sobre as pretas e entre duas cartas de um naipe fixo tenham apenas cartas deste mesmo naipe.

\section{2}

\section{Tirando vantagem da monotonicidade}

Devido à completa simetria de $S_{N}$, colocar ordem no conjunto das permutações parece ser uma ideia estranha e muito complicada. Assim, daremos um papel especial para a identidade, a qual fixamos como o elemento maximal em um certo sentido, e isso nos ajudará a quebrar esta simetria.

Antes de definirmos nossa ordem em $S_{N}$, vamos introduzir uma aplicação que leva permutações em superfícies discretas. 
Capítulo 5. O embaralhamento de transposições adjacentes visto em tempo contínuo

\subsection{1}

\section{Mapeando permutações em superfícies discretas}

Definição 5.1 À cada $\sigma \in S_{N}$ associamos a função $\tilde{\sigma}:\{0, \cdots, N\}^{2} \rightarrow \mathbb{R}$ definida por

$$
\tilde{\sigma}(x, y):=\sum_{z=1}^{x} \boldsymbol{1}_{\{\sigma(z) \leq y\}}-\frac{x y}{N} .
$$

Note que o termo $x y / N$ é subtraido para que $\tilde{\sigma}(x, y)$ tenha média zero com respeito à medida de equilíbrio $\pi$.

De fato,

$$
\begin{aligned}
\mathbb{E}_{\pi}[\tilde{\sigma}(x, y)] & =\mathbb{E}_{\pi}\left[\sum_{z=1}^{x} \mathbf{1}_{\{\sigma(z) \leq y\}}\right]-\frac{x y}{N} \\
& =\left(\sum_{z=1}^{x} \mathbb{P}_{\pi}[\sigma(z) \leq y]\right)-\frac{x y}{N}=\left(\sum_{z=1}^{x} \frac{y}{N}\right)-\frac{x y}{N}=0 .
\end{aligned}
$$

Proposição 5.2 A aplicação supracitada é injetiva.

Prova. De fato,

$$
\begin{aligned}
& \tilde{\sigma}(x, y)-\tilde{\sigma}(x, y-1)-\tilde{\sigma}(x-1, y)+\tilde{\sigma}(x-1, y-1)+\frac{1}{N} \\
& =\sum_{z=1}^{x} \mathbf{1}_{\{\sigma(z) \leq y\}}-\frac{x y}{N}-\sum_{z=1}^{x} \mathbf{1}_{\{\sigma(z) \leq y-1\}}+\frac{x(y-1)}{N} \\
& -\sum_{z=1}^{x-1} \mathbf{1}_{\{\sigma(z) \leq y\}}+\frac{(x-1) y}{N}+\sum_{z=1}^{x-1} \mathbf{1}_{\{\sigma(z) \leq y-1\}}-\frac{(x-1)(y-1)}{N}+\frac{1}{N} \\
& =\sum_{z=1}^{x}\left[\mathbf{1}_{\{\sigma(z) \leq y\}}-\mathbf{1}_{\{\sigma(z) \leq y-1\}}\right]-\sum_{z=1}^{x-1}\left[\mathbf{1}_{\{\sigma(z) \leq y\}}-\mathbf{1}_{\{\sigma(z) \leq y-1\}}\right] \\
& =\sum_{z=1}^{x} \mathbf{1}_{\{\sigma(z)=y\}}-\sum_{z=1}^{x-1} \mathbf{1}_{\{\sigma(z)=y\}}=\mathbf{1}_{\{\sigma(x)=y\}} .
\end{aligned}
$$

Assim, se $\tilde{\sigma_{1}}=\tilde{\sigma_{2}}$, então para todos $x, y \in\{0, \cdots, N\}, \mathbf{1}_{\left\{\sigma_{1}(x)=y\right\}}=$ $\mathbf{1}_{\left\{\sigma_{2}(x)=y\right\}}$. Logo, $\sigma_{1}=\sigma_{2}$.

Identificaremos o conjunto imagem $\left\{\tilde{\sigma}: \sigma \in S_{N}\right\}$ com $S_{N}$ desde que isso não gere confusão. 
Capítulo 5. O embaralhamento de transposições adjacentes visto em tempo contínuo

Definição 5.3 Definimos uma relação de ordem natural (parcial) em $S_{N}$ por

$$
\sigma \leq \sigma^{\prime} \Leftrightarrow \forall x, y, \tilde{\sigma}(x, y) \leq \tilde{\sigma}^{\prime}(x, y)
$$

Proposição 5.4 A identidade, que denotamos por e, é o elemento maximal de $\left(S_{N}, \geq\right)$, e a permutação $\sigma_{\min }$ definida por

$$
\forall x \in\{1, \cdots, N\}, \sigma_{\min }(x):=N+1-x
$$

é o elemento minimal.

Prova. Primeiro, notemos que $\tilde{e}(x, y)+\frac{x y}{N}=\sum_{z=1}^{x} \mathbf{1}_{\{z \leq y\}}=\min \{x, y\}$.

Se $x \leq y$, então

$$
\tilde{e}(x, y)+\frac{x y}{N}=x=\sum_{z=1}^{x} 1 \geq \sum_{z=1}^{x} \mathbf{1}_{\{\sigma(z) \leq y\}}=\tilde{\sigma}(x, y)+\frac{x y}{N} .
$$

Logo, $\tilde{e}(x, y) \geq \tilde{\sigma}(x, y)$. Indiferentemente, se $x>y$, então, como há no máximo $y-1$ cartas com marcadores menores que $y$,

$$
\tilde{\sigma}(x, y)+\frac{x y}{N}=\sum_{z=1}^{x} \mathbf{1}_{\{\sigma(z) \leq y\}} \leq y=\tilde{e}(x, y)+\frac{x y}{N}
$$

Assim, $\tilde{e}(x, y) \geq \tilde{\sigma}(x, y)$, e portanto $e$ é o elemento maximal de $\left(S_{N}, \geq\right)$. A prova de que $\sigma_{\min }$ é o elemento minimal é análoga.

\section{2 .2}

\section{A construção gráfica}

Apresentaremos agora uma construção da dinâmica que nos permite construir todas as trajetórias $\sigma_{t}^{\xi}$, começando com condição inicial $\xi \in S_{N}$, simultaneamente (um grande acoplamento e tem a propriedade de conservar ordem).

Associamos à cada $x \in\{1, \cdots, N-1\}$ um processo de Poisson independente $\left(\mathcal{T}^{x}\right)=\left(\mathcal{T}_{n}^{x}\right)_{n \geq 0}$ com intensidade dois.

Em outras palavras, $\mathcal{T}_{0}^{x}=0$ para todo $x \mathrm{e}$

$$
\left(\mathcal{T}_{n}^{x}-\mathcal{T}_{n-1}^{x}\right)_{x \in\{1, \cdots, N-1\}, n \geq 1}
$$

é um campo de variáveis aleatórias exponenciais i.i.d. com média $1 / 2$. 
Capítulo 5. O embaralhamento de transposições adjacentes visto em tempo contínuo

Referimo-nos a $\mathcal{T}=\left(\mathcal{T}^{x}\right)_{1 \leq x \leq N-1}$ como o processo do relógio. Note que o conjunto de valores tomados pelo processo do relógio é quase certamente um subconjunto discreto de $\mathbb{R}$, e como consequência, a probabilidade de que dois relógios toquem simultaneamente é zero.

Seja $\left(U_{n}^{x}\right)_{x \in\{1, \cdots, N-1\}, n \geq 1}$ um campo de variáveis aleatórias Bernoulli i.i.d. $\left(U_{n}^{x} \in\{0,1\}\right)$ com parâmetro $1 / 2$, independentes de $\mathcal{T}$. Agora, dados $\mathcal{T}$ e $U$, construiremos a trajetória da cadeia de Markov começando em $\xi \in S_{N}$.

A trajetória $\left(\sigma_{t}^{\xi}\right)_{t \geq 0}$ é càdlàg (contínua à direita com limite à esquerda) e é constante em intervalos onde o processo do relógio é silencioso.

Quando um relógio toca, isto é, no tempo $t=\mathcal{T}_{n}^{x}(n \geq 1)$, $\sigma_{t}^{\xi}$ é construída atualizando $\sigma_{t-}^{\xi}$ como abaixo:

- Se $\sigma_{t-}(x+1) \leq \sigma_{t-}(x)$ e $U_{n}^{x}=1$, ou $\sigma_{t-}(x+1) \geq \sigma_{t-}(x)$ e $U_{n}^{x}=0$, então nós trocamos os valores de $\sigma_{t-}(x)$ e $\sigma_{t-}(x+1)$;

- Em outros casos, não fazemos nada.

Em outras palavras, quando o processo do relógio associado a $x$ toca, nós ordenamos as cartas das posições $x$ e $x+1$ se $U_{n}^{x}=1$, e as desordenamos se $U_{n}^{x}=0$.

É imediato verificar que essa construção nos fornece uma cadeia de Markov com gerador $\mathcal{L}$ descrito em (5.1).

O efeito da atualização em $\tilde{\sigma}$ é o seguinte: Para cada $y \in\{1, \cdots, N-1\}$, se $\left(\tilde{\sigma}_{t-}(z, y)\right)_{z \in\{1, \cdots, N-1\}}$ apresenta um mínimo local em $z=x$ e $U_{n}^{x}=1$, então esse é transformado em um máximo local, isto é, $\tilde{\sigma}_{t}(x, y)=\tilde{\sigma}_{t-}(x, y)+1$. Do contrário, se apresenta um máximo local em $z=x$ e $U_{n}^{x}=0$, então $\tilde{\sigma}_{t}(x, y)=\tilde{\sigma}_{t-}(x, y)-1$. Chamamos esta operação de atualização de $\sigma$ na coordenada $x$.

Agora mostraremos que essa construção preserva a ordem.

Proposição 5.5 Seja $\xi \geq \xi^{\prime}$ dois elementos de $S_{N}$. Com a construção gráfica acima, temos $\sigma_{t}^{\xi} \geq \sigma_{t}^{\xi^{\prime}}$.

Prova. A demonstração que segue encontra-se em [11].

A única coisa a se verificar é que a ordem é conservada cada vez que o processo do relógio toca, isto é, para todo $(n, x)$ e $t=\mathcal{T}_{n}^{x}$,

$$
\sigma_{t-}^{\xi} \geq \sigma_{t-}^{\xi^{\prime}} \Rightarrow \sigma_{t}^{\xi} \geq \sigma_{t}^{\xi^{\prime}}
$$


Capítulo 5. O embaralhamento de transposições adjacentes visto em tempo contínuo

Suponhamos que $\sigma_{t-}^{\xi} \geq \sigma_{t-}^{\xi^{\prime}}$. Mostraremos que para todo $y \in$ $\{1, \cdots, N-1\}, \quad \tilde{\sigma}_{t}^{\xi}(x, y) \geq \tilde{\sigma}_{t}^{\xi^{\prime}}(x, y)$, o que implica o resultado já que as outras coordenadas não são trocadas no tempo $t$.

Com efeito, fixemos $y \in\{1, \cdots, N-1\}$. Notemos que quando $\tilde{\sigma}_{t-}^{\xi}(x, y)>$ $\tilde{\sigma}_{t-}^{\xi^{\prime}}(x, y)$, não precisamos fazer nada, pois não é possível que $\tilde{\sigma}^{\xi}$ pule para baixo enquanto $\tilde{\sigma}^{\xi^{\prime}}$ pula para cima.

Por esse motivo, vamos assumir que $\tilde{\sigma}_{t-}^{\xi}(x, y)=\tilde{\sigma}_{t-}^{\xi^{\prime}}(x, y)$. Se $U_{n}^{x}=1$, apenas temos que verificar que se $\tilde{\sigma}_{t}^{\xi^{\prime}}(x, y)$ pula para cima, então $\tilde{\sigma}_{t}^{\xi}(x, y)$ também o faz. Isso ocorre porque se $\tilde{\sigma}_{t-}^{\xi^{\prime}}(., y)$ apresenta um mínimo local em $x$, então $\tilde{\sigma}_{t-}^{\xi}(., y)$ também o faz. Analogamente, se $U_{n}^{x}=0$ e $\tilde{\sigma}_{t}^{\xi^{\prime}}(x, y)$ pula para baixo então $\tilde{\sigma}_{t}^{\xi}(x, y)$ também o faz.

\section{2 .3}

\section{Ordenação estocástica e sua preservação}

Definição 5.6 Sejam $\alpha, \beta$ duas medidas de probabilidade em um conjunto finito e ordenado $\Omega$. Dizemos que $\alpha$ domina $\beta$ estocasticamente e escrevemos $\alpha \succeq \beta$ se podemos encontrar um acoplamento $\Pi$, isto é, uma probabilidade em $\Omega^{2}$ tal que a primeira marginal tem lei $\alpha$ e a segunda $\beta$, e que satisfaz

$$
\Pi\left\{\left(\omega_{1}, \omega_{2}\right) \in \Omega^{2}: \omega_{1} \geq \omega_{2}\right\}=1
$$

Definição 5.7 Dizemos que uma função $f$ em $\Omega$ é crescente se $\forall \omega, \omega^{\prime} \in \Omega, \omega \geq$ $\omega^{\prime} \Rightarrow f(\omega) \geq f\left(\omega^{\prime}\right)$.

Definição 5.8 Para um conjunto ordenado $\Omega$, dizemos que um subconjunto $A$ (um evento) é crescente se a função $\mathbf{1}_{A}$ é crescente ou equivalentemente, se $\forall \omega \in A, \omega \geq \omega^{\prime} \Rightarrow \omega^{\prime} \in A$.

Agora daremos uma caracterização equivalente para a dominação estocástica.

Usaremos a notação $\alpha(f)$ para a esperança de $f$ com respeito à $\alpha$.

Lema 5.9 Sejam $\alpha$ e $\beta$ duas medidas de probabilidade em um conjunto finito e ordenado $\Omega$. As seguintes afirmações são equivalentes:

(i) $\alpha$ domina $\beta$;

(ii) Para toda função crescente $f$ definida em $\Omega, \alpha(f) \geq \beta(f)$. 
Capítulo 5. O embaralhamento de transposições adjacentes visto em tempo contínuo

Prova. Seja $f$ uma função crescente definida em $\Omega$. Se $\alpha \succeq \beta$, então existem variáveis aleatórias $X, Y$ com leis $\alpha, \beta$, respectivamente, tais que $\mathbb{P}[X>z] \geq \mathbb{P}[Y>z], \forall z \in \mathbb{R}$.

Assim,

$$
\mathbb{P}[f(X)>z]=\mathbb{P}\left[X>f^{-1}(z)\right] \geq \mathbb{P}\left[Y>f^{-1}(z)\right]=\mathbb{P}[f(Y)>z]
$$

Se $f(X), f(Y) \geq 0$, então

$\alpha(f)=\mathbb{E}[f(X)]=\int_{0}^{\infty} \mathbb{P}[f(X)>t] d t \geq \int_{0}^{\infty} \mathbb{P}[f(Y)>t] d t=\mathbb{E}[f(Y)]=\beta(f)$.

Se não temos $f(X) \geq 0$ e $f(Y) \geq 0$, então

$$
\begin{aligned}
\alpha(f) & =\mathbb{E}[f(X)]=\int_{0}^{\infty} d t-\int_{-\infty}^{\infty} \mathbb{P}[f(X) \leq t] d t \\
& \geq \int_{0}^{\infty} d t-\int_{-\infty}^{\infty} \mathbb{P}[f(Y) \leq t] d t \\
& =\mathbb{E}[f(Y)]=\beta(f) .
\end{aligned}
$$

Reciprocamente, para cada $z \in \mathbb{R}$, definamos $f_{z}(X):=\mathbf{1}_{\{X>z\}}$, que é monótona.

Assim,

$$
\mathbb{P}[X>z]=\mathbb{E}\left[f_{z}(X)\right]=\alpha\left(f_{z}\right) \geq \beta\left(f_{z}\right)=\mathbb{E}\left[f_{z}(Y)\right]=\mathbb{P}[Y>z]
$$

Logo, $\alpha \succeq \beta$.

Uma consequência da Proposição 5.5 é que se $\nu$ e $\nu^{\prime}$ são medidas de probabilidade em $S_{N}$, então

$$
\nu \succeq \nu^{\prime} \Rightarrow \forall t \geq 0, P_{t}^{\nu} \succeq P_{t}^{\nu^{\prime}} .
$$

Agora vamos introduzir uma simples ferramenta para produzir acoplamentos estocásticos.

Lema 5.10 Seja $\Omega$ um conjunto finito e $\left(\omega_{t}^{1}\right)_{t>0} e\left(\omega_{t}^{2}\right)_{t>0}$ dois processos estocásticos em $\Omega$. Assuma que as distribuições de $\omega_{t}^{1}$ e $\omega_{t}^{2}$ convergem para duas medidas de probabilidade $\alpha$ e $\beta$, respectivamente, quando $t$ tende ao infinito. Se podemos encontrar um acoplamento dos processos tal que para todo $t \geq 0, \omega_{t}^{1} \geq \omega_{t}^{2}$ quase certamente, então $\alpha \succeq \beta$. 
Capítulo 5. O embaralhamento de transposições adjacentes visto em tempo contínuo

Prova. A demonstração que segue encontra-se em [11].

Seja $\Pi_{t}$ a lei de $\left(\omega_{t}^{1}, \omega_{t}^{2}\right)$ sob o acoplamento dado pelo lema.

Para todo $t \geq 0, \Pi_{t}$ é suportado no conjunto

$$
\mathcal{D}=\left\{\left(\omega^{1}, \omega^{2}\right) \in \Omega^{2}: \omega^{1} \geq \omega^{2}\right\} .
$$

Como $\Pi_{t}$ mora num espaço compacto (pela topologia induzida pela distância de variação total), ela tem pelo menos um ponto limite que chamaremos de $\Pi$ e é suportado em $\mathcal{D}$. A medida $\Pi$ gera um acoplamento que satisfaz $\alpha \succeq \beta$.

\subsection{4}

\section{Desigualdades de correlação e a desigualdade FKG}

Umas das importantes ferramentas que usaremos são as desigualdades de correlação, que dizem que condicionar $\pi$ em um evento crescente faz todos os outros eventos crescentes mais prováveis.

Lema 5.11 Sejam $f$ e $g$ duas funções reais crescentes de uma variável e $X$ uma variável aleatória com lei $\mathbb{P}$. Então,

$$
\mathbb{E}[f(X) g(X)] \geq \mathbb{E}[f(X)] \mathbb{E}[g(X)]
$$

Prova. Seja $X^{\prime}$ uma cópia independente de $X$, que existe pelo Teorema de Kolmogorov (veja o Teorema 2.1.14. na página 51 de [6]). Então, como $f$ e $g$ são crescentes, temos que

$$
\begin{aligned}
0 & \leq \mathbb{E}\left[\left(f(X)-f\left(X^{\prime}\right)\right)\left(g(X)-g\left(X^{\prime}\right)\right)\right] \\
& =\mathbb{E}\left[f(X) g(X)-f(X) g\left(X^{\prime}\right)-f\left(X^{\prime}\right) g(X)+f\left(X^{\prime}\right) g\left(X^{\prime}\right)\right] \\
& =\mathbb{E}[f(X) g(X)]-\mathbb{E}\left[f(X) g\left(X^{\prime}\right)\right]-\mathbb{E}\left[f\left(X^{\prime}\right) g(X)\right]+\mathbb{E}\left[f\left(X^{\prime}\right) g\left(X^{\prime}\right)\right] \\
& =2 \mathbb{E}[f(X) g(X)]-2 \mathbb{E}[f(X)] \mathbb{E}[g(X)] .
\end{aligned}
$$

Portanto,

$$
\mathbb{E}[f(X) g(X)] \geq \mathbb{E}[f(X)] \mathbb{E}[g(X)]
$$

A desigualdade (5.3) não é verdadeira em geral para todas as noções de ordem parcial, mas existe uma generalização dela para "lattices distributi- 
Capítulo 5. O embaralhamento de transposições adjacentes visto em tempo contínuo

vos"conhecida por desigualdade FKG (Fortuin-Kasteleyn-Ginibre).

Infelizmente, $S_{N}$ não é um lattice distributivo. Mais precisamente, se definirmos para $\sigma$ e $\sigma^{\prime}$ em $S_{N}, \min \left(\tilde{\sigma}, \tilde{\sigma}^{\prime}\right)$ e $\max \left(\tilde{\sigma}, \tilde{\sigma^{\prime}}\right)$ por

$$
\min \left(\tilde{\sigma}, \tilde{\sigma}^{\prime}\right)(x, y):=\min \left(\tilde{\sigma}(x, y), \tilde{\sigma}^{\prime}(x, y)\right)
$$

e

$$
\max \left(\tilde{\sigma}, \tilde{\sigma}^{\prime}\right)(x, y):=\max \left(\tilde{\sigma}(x, y), \tilde{\sigma}^{\prime}(x, y)\right),
$$

então $\min \left(\tilde{\sigma}, \tilde{\sigma}^{\prime}\right)$ e $\max \left(\tilde{\sigma}, \tilde{\sigma}^{\prime}\right)$ não são necessariamente imagens de elementos de $S_{N}$. Porém, em [11], é feita uma adaptação da prova escrita em [9] para o nosso caso.

Proposição 5.12 (A desigualdade FKG para permutações) Para qualquer par de funções crescentes $f$ e $g$ definidas em $S_{N}$,

$$
\pi(f(\sigma) g(\sigma)) \geq \pi(f(\sigma)) \pi(g(\sigma)) .
$$

Prova.

Primeiro, vejamos que para qualquer par $(A, B)$ de conjuntos crescentes, temos $\pi(A \cap B) \geq \pi(A) \pi(B)$.

De fato, sejam $A$ e $B$ dois subconjuntos crescentes de $S_{N}$. Vamos começar da identidade e iterar duas dinâmicas acopladas $\sigma_{t}$ e $\sigma_{t}^{A}$ definidas da seguinte maneira: $\sigma_{t}$ é um embaralhamento de transposições adjacentes normal e $\sigma_{t}^{A}$ tem a mesma regra de transição exceto que todas as transições que saem de $A$ são canceladas (isso é chamado de cadeia de Markov refletida).

Acoplamos as duas dinâmicas usando a construção gráfica feita da seção 5.2.2, com ambas as dinâmicas usando o mesmo processo do relógio $\mathcal{T}$ e variáveis de atualização $U$, a única diferença sendo o fato de que $\sigma_{t}^{A}$ cancela as transições que fazem a cadeia sair de $A$.

A cadeia de Markov $\sigma_{t}^{A}$ é irredutível. A razão para isso ocorrer é o fato de que para cada $\left(\sigma, \sigma^{\prime}\right) \in A^{2}$, podemos sempre encontrar uma sequência de transições que vão para cima (ordenando vizinhos) que leva $\sigma$ até $e$ (a identidade) e uma sequência de transições que vão para baixo e que leva $e$ até $\sigma^{\prime}$. A concatenação destas duas sequências nos fornece um caminho de transições de $\sigma$ até $\sigma^{\prime}$ cujos passos estão todos em $A$ (elas são $\geq \sigma$ na primeira parte e $\geq \sigma^{\prime}$ na segunda parte). 
Capítulo 5. O embaralhamento de transposições adjacentes visto em tempo contínuo

Além disso, $\pi(. \mid A)$, isto é, a medida uniforme em $A$, é reversível para $\sigma^{A}$, pois

$$
\pi(\sigma \mid A) \cdot P_{t}\left(\sigma, \sigma^{\prime}\right)=\pi\left(\sigma^{\prime} \mid A\right) \cdot P_{t}\left(\sigma^{\prime}, \sigma\right), \forall \sigma, \sigma^{\prime} \in A .
$$

Portanto, $\sigma_{t}^{A}$ converge para $\pi(. \mid A)$.

Como as únicas transições que são canceladas em $\sigma^{A}$ são as transições que "descem- correspondendo a desordenação de um par adjacente -, temos como consequência da Proposição 5.5 que

$$
\forall t \geq 0, \sigma_{t}^{A} \geq \sigma_{t}
$$

Usando o Lema 5.10, obtemos $\pi(. \mid A) \succeq \pi$. Logo, $\pi(B \mid A) \geq \pi(B)$. $\mathrm{E}$ portanto, $\pi(A \cap B) \geq \pi(A) \pi(B)$.

Agora, sejam $f$ e $g$ duas funções positivas e crescentes (não há perda de generalidade em assumir positividade pois adicionar uma constante em $f$ ou $g$ não altera a desigualdade proposta) e $x, y \in \mathbb{R}$.

Definimos os seguintes conjuntos crescentes:

$$
A_{x}:=\{f(\sigma) \geq x\} \text { e } B_{y}:=\{g(\sigma) \geq y\} .
$$

Como $f=\int_{\mathbb{R}} \mathbf{1}_{A_{x}} d x$ e $g=\int_{\mathbb{R}} \mathbf{1}_{B_{y}} d y$,

$$
\begin{aligned}
\pi(f(\sigma) g(\sigma)) & =\pi\left(\int_{\mathbb{R}^{2}} \mathbf{1}_{A_{x}} \mathbf{1}_{B_{y}} d x \cdot d y\right)=\int_{\mathbb{R}^{2}} \pi\left(A_{x} \cap B_{y}\right) d x \cdot d y \\
& \geq \int_{\mathbb{R}^{2}} \pi\left(A_{x}\right) \pi\left(B_{y}\right) d x \cdot d y=\left(\int_{\mathbb{R}} \pi\left(A_{x}\right) d x\right)\left(\int_{\mathbb{R}} \pi\left(B_{y}\right) d y\right) \\
& =\left(\int_{\mathbb{R}} \pi\left(\mathbf{1}_{A_{x}}\right) d x\right)\left(\int_{\mathbb{R}} \pi\left(\mathbf{1}_{B_{y}}\right) d y\right)=\pi\left(\int_{\mathbb{R}} \mathbf{1}_{A_{x}} d x\right) \pi\left(\int_{\mathbb{R}} \mathbf{1}_{B_{y}} d y\right) \\
& =\pi(f(\sigma)) \pi(g(\sigma)) .
\end{aligned}
$$

\section{2 .5}

\section{A desigualdade censuradora}

A desigualdade censuradora, um resultado estabelecido em [15] para "sistemas monótonos", é uma noção que levemente generaliza a dinâmica de Glauber para sistemas de spins com um espaço de spins totalmente ordenado e diz que cancelar algumas das atualizações dos spins não tem o efeito de atrasar muito a mistura. Infelizmente, o embaralhamento de transposições adjacentes não é um 
Capítulo 5. O embaralhamento de transposições adjacentes visto em tempo contínuo

sistema monótono no sentido de [15]. Todavia, em [11], é feita uma adaptação da prova do resultado supracitado para o nosso setup. Antes de apresentarmos o resultado, vamos introduzir um pouco de terminologia e notação.

Definição 5.13 Um esquema censurador é uma função càdlàg $\mathcal{C}: \mathbb{R} \rightarrow$ $\mathcal{P}(\{1, \cdots, N-1\})$ onde $\mathcal{P}(S)$ é o conjunto das partes de $S$.

A dinâmica censurada com esquema $\mathcal{C}$ é a dinâmica obtida usando a construção gráfica descrita na seção 5.2 .2 exceto que se $\mathcal{T}^{x}$ toca em tempo $t$, a atualização é feita se, e somente se, $x \in \mathcal{C}(t)$.

É natural pensar que a cada vez que o relógio toca, $\sigma_{t}$ estará mais perto do equilíbrio e portanto a censura apenas fará a convergência ao equilíbrio mais lenta. A desigualdade censuradora garante que isso é verdade se começarmos com uma medida cuja densidade é uma função crescente.

Dados um esquema censurador $\mathcal{C}$ e uma distribuição de probabilidade $\nu$ em $S_{N}$, seja $P_{t}^{\nu, \mathcal{C}}$ a distribuição de $\sigma_{t}$ que realizou a dinâmica censurada até tempo $\mathrm{t}$ começando com distribuição inicial $\nu$.

Definição 5.14 Dizemos que uma lei de probabilidade $\nu$ em $S_{N}$ é crescente se $\sigma \mapsto \nu(\sigma)$ é uma função crescente.

Proposição 5.15 (Desigualdade censuradora) Se $\nu$ é crescente, então para todo $t \geq 0$,

$$
\left\|P_{t}^{\nu, \mathcal{C}}-\pi\right\|_{T V} \geq\left\|P_{t}^{\nu}-\pi\right\|_{T V} .
$$

Prova. Dados $x \in\{1, \cdots, N-1\}$ e $\sigma \in S_{N}$, definimos

$$
\sigma_{x}^{\bullet}:=\left\{\xi \in S_{N} ; \forall y \notin\{x, x+1\}, \xi(y)=\sigma(y)\right\} .
$$

O conjunto $\sigma_{x}^{\bullet}$ contém dois elementos (sendo $\sigma$ um deles) $\sigma_{x}^{+} \geq \sigma_{x}^{-}$, que são obtidos respectivamente ordenando e desordenando $\sigma(x)$ e $\sigma(x+1)$. Dada $\nu$ uma medida de probabilidade em $S_{N}$, definimos $\theta_{x}(\nu)$, a medida "atualizada em $x$ " por

$$
\theta_{x}(\nu)(\sigma):=\frac{\nu\left(\sigma_{x}^{\bullet}\right)}{2}
$$


Capítulo 5. O embaralhamento de transposições adjacentes visto em tempo contínuo

O operador $\theta_{x}$ descreve como a lei de $\sigma_{t}$ é mudada quando o processo do relógio toca em $x$.

Afirmação 1: Se $\nu$ é crescente, então $\theta_{x}(\nu)$ também é, e além disso, $\nu \succeq \theta_{x}(\nu)$.

De fato, é fácil verificar que se $\sigma \geq \xi$, então $\sigma_{x}^{+} \geq \xi_{x}^{+}$e $\sigma_{x}^{-} \geq \xi_{x}^{-}$. Logo, se $\nu$ é crescente, temos que

$$
\nu\left(\sigma_{x}^{\bullet}\right)=\nu\left(\sigma_{x}^{+}\right)+\nu\left(\sigma_{x}^{-}\right) \geq \nu\left(\xi_{x}^{+}\right)+\nu\left(\xi_{x}^{-}\right)=\nu\left(\xi_{x}^{\bullet}\right),
$$

e portanto, $\theta_{x}(\nu)$ é crescente.

Agora, seja $g$ uma função crescente definida no espaço de estados $\Omega_{N}$ com valores em $\mathbb{R}$, e suponhamos que $\nu$ é crescente. Então, $\nu\left(\sigma_{x}^{+}\right) \geq \nu\left(\sigma_{x}^{-}\right)$e $g\left(\sigma_{x}^{+}\right) \geq g\left(\sigma_{x}^{-}\right)$. Assim,

$$
\begin{aligned}
0 & \leq\left[g\left(\sigma_{x}^{+}\right)-g\left(\sigma_{x}^{-}\right)\right]\left[\nu\left(\sigma_{x}^{+}\right)-\nu\left(\sigma_{x}^{-}\right)\right] \\
& =\left[g\left(\sigma_{x}^{+}\right) \nu\left(\sigma_{x}^{+}\right)+g\left(\sigma_{x}^{-}\right) \nu\left(\sigma_{x}^{-}\right)\right]-\left[g\left(\sigma_{x}^{+}\right) \nu\left(\sigma_{x}^{-}\right)+g\left(\sigma_{x}^{-}\right) \nu\left(\sigma_{x}^{+}\right)\right] .
\end{aligned}
$$

Logo,

$$
\left[g\left(\sigma_{x}^{+}\right) \nu\left(\sigma_{x}^{-}\right)+g\left(\sigma_{x}^{-}\right) \nu\left(\sigma_{x}^{+}\right)\right] \leq\left[g\left(\sigma_{x}^{+}\right) \nu\left(\sigma_{x}^{+}\right)+g\left(\sigma_{x}^{-}\right) \nu\left(\sigma_{x}^{-}\right)\right] .
$$

Portanto,

$$
\begin{aligned}
& {\left[g\left(\sigma_{x}^{+}\right) \nu\left(\sigma_{x}^{+}\right)+g\left(\sigma_{x}^{-}\right) \nu\left(\sigma_{x}^{-}\right)\right]} \\
& =\frac{\left[g\left(\sigma_{x}^{+}\right) \nu\left(\sigma_{x}^{+}\right)+g\left(\sigma_{x}^{-}\right) \nu\left(\sigma_{x}^{-}\right)\right]+\left[g\left(\sigma_{x}^{+}\right) \nu\left(\sigma_{x}^{+}\right)+g\left(\sigma_{x}^{-}\right) \nu\left(\sigma_{x}^{-}\right)\right]}{2} \\
& \geq \frac{\left[g\left(\sigma_{x}^{+}\right) \nu\left(\sigma_{x}^{+}\right)+g\left(\sigma_{x}^{-}\right) \nu\left(\sigma_{x}^{-}\right)\right]+\left[g\left(\sigma_{x}^{+}\right) \nu\left(\sigma_{x}^{-}\right)+g\left(\sigma_{x}^{-}\right) \nu\left(\sigma_{x}^{+}\right)\right]}{2} \\
& =\frac{\left[g\left(\sigma_{x}^{+}\right)+g\left(\sigma_{x}^{-}\right)\right]\left[\nu\left(\sigma_{x}^{+}\right)+\nu\left(\sigma_{x}^{-}\right)\right]}{2} \\
& =\left[g\left(\sigma_{x}^{+}\right)+g\left(\sigma_{x}^{-}\right)\right] \theta_{x}(\nu)(\sigma) \\
& =g\left(\sigma_{x}^{+}\right) \theta_{x}(\nu)(\sigma)+g\left(\sigma_{x}^{-}\right) \theta_{x}(\nu)(\sigma) .
\end{aligned}
$$

Agora, somando em $\sigma \in S_{N}$ e dividindo por dois, temos 
Capítulo 5. O embaralhamento de transposições adjacentes visto em tempo contínuo

$$
\nu(g)=\sum_{\sigma \in S_{N}} g(\sigma) \nu(\sigma) \geq \sum_{\sigma \in S_{N}} g(\sigma) \theta_{x}(\nu)(g(\sigma))=\theta_{x}(\nu)(g) .
$$

Como $g$ é arbitrária, temos que

$$
\nu \succeq \theta_{x}(\nu)
$$

Afirmação 2: Seja $\nu$ uma medida de probabilidade crescente em $S_{N}$. Então, para todo $t \geq 0, P_{t}^{\nu}$ também é crescente e para qualquer esquema censurador $\mathcal{C}$, $P_{t}^{\nu, \mathcal{C}}$ é crescente.

Seja $\nu$ uma probabilidade crescente e $\sigma_{t}^{\nu}$ a trajetória da cadeia de Markov obtida com a construção gráfica. Por definição, temos $P_{t}^{\nu}=\mathbb{P}\left[\sigma_{t}^{\nu} \in\right.$. $]$. Seja $\mathcal{N}$ o número de atualizações que ocorrem antes do tempo $t$ e $X_{1}, \cdots, X_{\mathcal{N}}$ a sequência de vértices que tocaram no processo do relógio (com repetições). Então, a lei de probabilidade $\mathbb{P}\left[\sigma_{t}^{\nu} \in \mid \cdot \mathcal{T}\right]$, sabendo que o processo do relógio é dado por

$$
\theta_{X_{\mathcal{N}}} \circ \cdots \circ \theta_{X_{1}}(\nu)
$$

é crescente de acordo com a Afirmação 1 .

A monotonicidade é então preservada quando fazemos a média com respeito à $\mathcal{T}$. O resultado é análogo para a dinâmica censurada.

Afirmação 3: As atualizações preservam dominação estocástica no sentido de que se $\nu_{1} \succeq \nu_{2}$, então $\theta_{x}\left(\nu_{1}\right) \succeq \theta_{x}\left(\nu_{2}\right)$.

A veracidade da afirmação segue da definição de $\theta_{x}$.

Afirmação 4: Se $\nu_{1}$ tem densidade crescente e $\nu_{1} \preceq \nu_{2}$, então

$$
\left\|\nu_{1}-\pi\right\|_{T V} \leq\left\|\nu_{2}-\pi\right\|_{T V}
$$

De fato, definamos $A:=\left\{\sigma ; \nu_{1}(\sigma) \geq \pi(\sigma)=(n !)^{-1}\right\}$.

Como $\nu_{1}$ tem densidade crescente, $A$ é um evento crescente e

$$
\left\|\nu_{1}-\pi\right\|_{T V}=\nu_{1}(A)-\pi(A) \leq \nu_{2}(A)-\pi(A)=\left\|\nu_{2}-\pi\right\|_{T V} .
$$

Afirmação 5: Seja $\nu_{0}$ uma probabilidade crescente em $S_{N}$ e $k \in \mathbb{N}$. Dados $\left(x_{1}, \cdots, x_{k}\right) \in\{1, \cdots, N-1\}^{k}$ (repetições são permitidas) e $j \in\{1, \cdots, k\}$, 
Capítulo 5. O embaralhamento de transposições adjacentes visto em tempo contínuo

denotemos por $\nu_{1}$ a medida obtida realizando sucessivas atualizações nos sítios $x_{1}, \cdots, x_{k}$ e $\nu_{2}$ a medida obtida realizando a mesma sequência de atualizações mas omitindo a atualização em $x_{j}$ (isto é, $x_{1}, \cdots, x_{j-1}, x_{j+1}, x_{j+2}, \cdots, x_{k}$ ). Então,

$$
\left\|\nu_{1}-\pi\right\|_{T V} \geq\left\|\nu_{2}-\pi\right\|_{T V} .
$$

Observação: O resultado permanece válido se várias atualizações são omitidas ao invés de uma.

De fato, sem perda de generalidade podemos considerar que $j=1$ pois a lei obtida depois de realizar as $j-1$ primeiras atualizações tem densidade crescente.

Seja $\nu_{0}^{\prime}$ a medida obtida depois de atualizar $x_{1}$. Da Afirmação 1 , temos que $\nu_{0}^{\prime} \preceq \nu_{0}$. Como a monotonicidade é preservada pelas atualizações em $\left(x_{2}, \cdots, x_{k}\right)$, a Afirmação 3 garante que $\nu_{2} \preceq \nu_{1}$. Além disso, pela Afirmação 1 , ambas possuem densidades crescentes. Portanto, a Afirmação 4 garante o resultado.

O caso de várias omissões pode ser provado usando o princípio de indução.

Prova da proposição: Finalmente, vamos mostrar a desigualdade censuradora. Na nossa dinâmica, em tempo $t$, o conjunto das atualizações realizadas é aleatório e dado pelo processo do relógio $\mathcal{T}$ restrito a $[0, t]$, logo a Afirmação 5 não pode ser aplicada diretamente. Todavia, para uma realização fixa de $\mathcal{T}$, podemos aplicar a Afirmação 5 condicionada a $\mathcal{T}$. Com efeito, sejam $P_{t}^{\mathcal{T}}:=\mathbb{P}\left[\sigma_{t}^{\nu} \in . \mid \mathcal{T}\right]$ a lei de $\sigma$ obtida depois de fazer as atualizações correspondentes a $\mathcal{T}$, e $P_{t}^{\mathcal{T}, \mathcal{C}}:=\mathbb{P}^{\mathcal{C}}\left[\sigma_{t}^{\nu} \in . \mid \mathcal{T}\right]$ a lei obtida depois de fazer as atualizações permitidas pelo esquema censurador. Ambas medidas de probabilidade são $i$ ) crescentes, e pela Afirmação $5, i i) P^{\mathcal{T}} \succeq P^{\mathcal{T}, \mathcal{C}}$.

Essas duas propriedades são conservadas quando fazemos a média com respeito a $\mathcal{T}$. Logo, $P_{t}^{\nu} \succeq P_{t}^{\nu, \mathcal{C}}$ e portanto a Afirmação 4 garante o resultado.

A desigualdade censuradora tem sido usada em uma variedade de contextos para cotar tempos de mistura para cadeias de Markov. A estratégia geralmente é encontrar um esquema censurador que nos permita controlar melhor a dinâmica sem reduzir muito sua velocidade (LACOIN, 2016, p. 15). 
Capítulo 5. O embaralhamento de transposições adjacentes visto em tempo contínuo

\subsection{6}

\section{Projeção e monotonia}

Agora mostraremos que se $\nu$ é uma medida de probabilidade crescente em $S_{N}$, então suas projeções têm densidades crescentes com respeito às projeções da medida invariante $\pi$.

Definição 5.16 Definimos o semi-esqueleto de uma permutação $\sigma \in S_{N}$ por $\hat{\sigma}:\{0, \ldots, N\} \times\{0, \ldots, K\} \rightarrow \mathbb{R}$ que faz

$$
\hat{\sigma}(x, j):=\tilde{\sigma}\left(x, x_{j}\right), \forall 0 \leq j \leq K
$$

onde $x_{j}:=\lceil j N / K\rceil$. Chamaremos de $\hat{S_{N}}$ o conjunto de semi-esqueletos adimissíveis, isto é, a imagem de $S_{N}$ por $\hat{\sigma}$.

Definição 5.17 Definimos o esqueleto de uma permutação $\sigma \in S_{N}$ por $\bar{\sigma}$ : $\{0, \ldots, K\}^{2} \rightarrow \mathbb{R}$ que faz

$$
\bar{\sigma}(i, j):=\tilde{\sigma}\left(x_{i}, x_{j}\right), \forall 0 \leq i, j \leq K
$$

Chamaremos de $\overline{S_{N}}:=\left\{\bar{\sigma} \mid \sigma \in S_{N}\right\}$ o conjunto de esqueletos adimissíveis.

Definição 5.18 Equiparemos $\overline{S_{N}}$ e $\hat{S_{N}}$ com a ordem natural, isto é,

$$
\bar{\sigma} \geq \bar{\sigma}^{\prime} \Leftrightarrow \bar{\sigma}(i, j) \geq \bar{\sigma}^{\prime}(i, j), \forall 0 \leq i, j \leq K,
$$

e

$$
\hat{\sigma} \geq \hat{\sigma}^{\prime} \Leftrightarrow \hat{\sigma}(x, j) \geq \hat{\sigma}^{\prime}(x, j), \forall 0 \leq x \leq N, 0 \leq j \leq K
$$

Notemos que quando $N=52$ e $K=2$, o semi-esqueleto codifica as posições das cartas vermelhas no baralho.

De fato, se $N=52$ e $K=2$, então $x_{1}=26$. Assim,

$$
\hat{\sigma}(x, 1)=\tilde{\sigma}(x, 26)=\sum_{z=1}^{x} \mathbf{1}_{\{\sigma(z) \leq 26\}}-\frac{x}{2} .
$$

Já o esqueleto indica o número de cartas vermelhas na primeira metade do baralho, pois

$$
\bar{\sigma}(1,1)+13=\tilde{\sigma}(26,26)+13=\sum_{z=1}^{26} \mathbf{1}_{\{\sigma(z) \leq 26\}} .
$$


Capítulo 5. O embaralhamento de transposições adjacentes visto em tempo contínuo

Definição 5.19 Seja $\nu$ uma medida de probabilidade em $S_{N}$. Chamaremos por $\bar{\nu}$ a medida imagem em $\overline{S_{N}}$ de $\nu$ pela projeção do esqueleto e por $\hat{\nu}$ a medida imagem do semi-esqueleto. Escreveremos $\bar{\nu}_{i, j}$ para a medida imagem de $\nu$ pela projeção $\sigma \mapsto \bar{\sigma}(i, j)$. Em particular $\bar{\pi}$ e $\bar{\pi}_{i, j}$ denotam as projeções da medida invariante.

Proposição 5.20 (Preservação da monotonia pela projeção) As seguintes afirmações são verdadeiras:

(i) Sejam $\bar{\sigma}^{1}, \bar{\sigma}^{2} \in \overline{S_{N}}$. Se $\bar{\sigma}^{1} \geq \bar{\sigma}^{2}$, então $\pi\left(\cdot \mid \bar{\sigma}=\bar{\sigma}^{1}\right) \succeq \pi\left(\cdot \mid \bar{\sigma}=\bar{\sigma}^{2}\right)$.

(ii) Dados $i, j \in\{1, \ldots, K\}$ e $z_{1} \geq z_{2}$ dois valores adimissíveis para $\bar{\sigma}(i, j)$, temos $\pi\left(\cdot \mid \bar{\sigma}(i, j)=z_{1}\right) \succeq \pi\left(\cdot \mid \bar{\sigma}(i, j)=z_{2}\right)$.

(iii) Se $\nu$ é uma medida de probabilidade crescente em $S_{N}$, então a densidade $\bar{\nu} / \bar{\pi}$ é uma função crescente em $\overline{S_{N}}$.

(iv) Se $\nu$ é uma medida de probabilidade crescente em $S_{N}$, então $\bar{\nu}_{i, j} / \bar{\pi}_{i, j}$ é uma função crescente no conjunto de valores adimissíveis para $\bar{\sigma}(i, j)$.

Prova. Dividiremos a prova em três afirmações.

Afirmação 1: Se $\hat{\sigma}^{1}, \hat{\sigma}^{2} \in \hat{S_{N}}$ e $\hat{\sigma}^{1} \geq \hat{\sigma}^{2}$, então

$$
\pi\left(\cdot \mid \hat{\sigma}=\hat{\sigma}^{1}\right) \succeq \pi\left(\cdot \mid \hat{\sigma}=\hat{\sigma}^{2}\right) .
$$

Notemos que a informação que $\hat{\sigma}$ carrega é exatamente o valor dos conjuntos

$$
\sigma^{-1}\left(\left\{x_{i-1}+1, \ldots, x_{i}\right\}\right), i \in\{1, \ldots, K\}
$$

Para cada $i \in\{0, \ldots, K\}$ esse conjunto é dado por

$$
\{x \in\{1, \ldots, N\} \mid \hat{\sigma}(x, i+1)-\hat{\sigma}(x-1, i+1)-\hat{\sigma}(x, i)+\hat{\sigma}(x-1, i)>0\},
$$

pois

$$
\begin{aligned}
\hat{\sigma}(x, i+1) & -\hat{\sigma}(x-1, i+1)-\hat{\sigma}(x, i)+\hat{\sigma}(x-1, i) \\
& =[\hat{\sigma}(x, i+1)-\hat{\sigma}(x-1, i+1)]-[\hat{\sigma}(x, i)-\hat{\sigma}(x-1, i)] \\
& =\left(\mathbf{1}_{\left\{\sigma(x) \leq x_{i+1}\right\}}-\frac{x_{i+1}}{N}\right)-\left(\mathbf{1}_{\left\{\sigma(x) \leq x_{i}\right\}}-\frac{x_{i}}{N}\right) \\
& =\mathbf{1}_{\left\{\sigma(x) \leq x_{i+1}\right\}}-\mathbf{1}_{\left\{\sigma(x) \leq x_{i}\right\}}-\underbrace{\frac{x_{i+1}-x_{i}}{N}}_{<1} \\
& \left\{\begin{array}{l}
>0 \quad \text { se } x \in\left\{x_{i-1}+1, \ldots, x_{i}\right\} ; \\
\leq 0 \quad \text { caso contrário. }
\end{array}\right.
\end{aligned}
$$


Capítulo 5. O embaralhamento de transposições adjacentes visto em tempo contínuo

Porém não temos informação sobre a ordem das cartas cujos marcadores pertencem a $\left\{x_{i-1}+1, \ldots, x_{i}\right\}$. Assim, para cada $\xi \in \hat{S_{N}}$, definimos a bijeção natural

$$
\begin{aligned}
\otimes_{i=1}^{K} S_{\Delta x_{i}} & \rightarrow\left\{\sigma \in S_{N} \mid \hat{\sigma}=\xi\right\}, \\
\left(\sigma_{1}, \ldots, \sigma_{K}\right) & \mapsto \sigma_{\xi}^{\left(\sigma_{1}, \ldots, \sigma_{K}\right)},
\end{aligned}
$$

onde $\Delta x_{i}:=x_{i}-x_{i-1}$, e a permutação $\sigma_{\xi}^{\left(\sigma_{1}, \ldots, \sigma_{K}\right)}$ é definida como a única em $\left\{\sigma \in S_{N} \mid \hat{\sigma}=\xi\right\}$ tal que para todo $i \in\{1, \ldots, K\}$, as cartas com marcadores $\left\{x_{i-1}, \ldots, x_{i}\right\}$ aparecem na ordem especificada por $\sigma_{i}$,

$$
\begin{gathered}
\forall a, b \in\left\{x_{i-1}+1, \ldots, x_{i}\right\} \\
\sigma^{-1}(a) \leq \sigma^{-1}(b) \Leftrightarrow \sigma_{i}^{-1}\left(a-x_{i-1}\right) \leq \sigma_{i}^{-1}\left(b-x_{i-1}\right) .
\end{gathered}
$$

A aplicação (5.4), em termos de superfícies discretas, tem a seguinte expressão: Para todo $y \in\left\{x_{i-1}, \ldots, x_{i}\right\}$

$$
\begin{aligned}
\tilde{\sigma}_{\xi}^{\left(\sigma_{1}, \ldots, \sigma_{K}\right)}(x, y) & =\frac{y-x_{i-1}}{\Delta x_{i}} \xi(x, i)+\frac{x_{i}-y}{\Delta x_{i}} \xi(x, i-1) \\
& +\tilde{\sigma}_{i}\left(\xi(x, i)-\xi(x, i-1)+\frac{x \Delta x_{i}}{N}, y-x_{i-1}\right)
\end{aligned}
$$

Se $\xi \geq \xi^{\prime}$ são dois semi-esqueletos admissíveis, então para qualquer $\left(\sigma_{1}, \ldots, \sigma_{K}\right)$,

$$
\tilde{\sigma}_{\xi}^{\left(\sigma_{1}, \ldots, \sigma_{K}\right)} \geq \tilde{\sigma}_{\xi^{\prime}}^{\left(\sigma_{1}, \ldots, \sigma_{K}\right)}
$$

De fato, sejam $x, y \in\left\{x_{i-1}, \ldots, x_{i}\right\}$,

$$
\begin{aligned}
\tilde{\sigma}_{\xi}^{\left(\sigma_{1}, \ldots, \sigma_{K}\right)}(x, y) & -\tilde{\sigma}_{\xi^{\prime}}^{\left(\sigma_{1}, \ldots, \sigma_{K}\right)}(x, y)=\frac{y-x_{i-1}}{\Delta x_{i}}\left[\xi(x, i)-\xi^{\prime}(x, i)\right] \\
& +\frac{x_{i}-y}{\Delta x_{i}}\left[\xi(x, i-1)-\xi^{\prime}(x, i-1)\right] \\
& +\tilde{\sigma}_{i}\left(\xi(x, i)-\xi(x, i-1)+\frac{x \Delta x_{i}}{N}, y-x_{i-1}\right) \\
& -\tilde{\sigma}_{i}\left(\xi^{\prime}(x, i)-\xi^{\prime}(x, i-1)+\frac{x \Delta x_{i}}{N}, y-x_{i-1}\right) \\
& \geq 0
\end{aligned}
$$

Portanto, a medida uniforme em $\otimes_{i=1}^{K} S_{\Delta_{x_{i}}}$ induz um acoplamento monótono que garante a veracidade de nossa afirmação.

Afirmação 2: Se $\bar{\sigma}^{1}, \bar{\sigma}^{2} \in \overline{S_{N}}$ e $\bar{\sigma}^{1} \geq \bar{\sigma}^{2}$, então $\pi\left(\cdot \mid \bar{\xi}=\bar{\sigma}^{1}\right) \succeq \pi\left(\cdot \mid \bar{\xi}=\bar{\sigma}^{2}\right)$. 
Capítulo 5. O embaralhamento de transposições adjacentes visto em tempo contínuo

De fato, sejam $\bar{\sigma}^{1} \geq \bar{\sigma}^{2}$ e para cada $i \in\{1,2\}$ definamos

$$
\hat{S}^{i}:=\left\{\xi \in \hat{S_{N}} \mid \bar{\xi}=\bar{\sigma}_{i}\right\}
$$

Mostraremos que $\hat{S}^{i}$ tem um elemento maximal $\xi_{\max }^{i}$ e que $\xi_{\max }^{1} \geq \xi_{\max }^{2}$.

Com efeito, seja $\sigma \in S_{N}$ tal que $\hat{\sigma} \in \hat{S}^{1}$ e seja $\sigma^{\prime}$ a permutação obtida ordenando os elementos de cada intervalo $\left\{x_{i-1}+1, \ldots, x_{i}\right\}$, para todo $i \in\{1, \ldots, K\}$, isto é, a única permutação que satisfaz

$$
\forall i \in\{1, \ldots, K\}, \sigma^{\prime}\left(\left\{x_{i-1}+1, \ldots, x_{i}\right\}\right)=\sigma\left(\left\{x_{i-1}+1, \ldots, x_{i}\right\}\right)
$$

e

$$
\begin{gathered}
\forall i \in\{1, \ldots, K\}, \forall y, z \in\left\{x_{i-1}+1, \ldots, x_{i}\right\} \\
y \leq z \Rightarrow \sigma^{\prime}(y) \leq \sigma^{\prime}(z)
\end{gathered}
$$

Assim, para todo $i \in\{1, \ldots, K\}, j \in\{0, \ldots, K\}$ e $x \in\left\{x_{i-1}, \ldots, x_{i}\right\}$, temos

$$
\hat{\sigma}^{\prime}(x, j):=\min \left(\frac{N-x_{j}}{N}\left(x-x_{i-1}\right)+\bar{\sigma}(i-1, j), \frac{x_{j}}{N}\left(x_{i}-x\right)+\bar{\sigma}(i, j)\right) .
$$

Portanto, $\hat{\sigma}^{\prime}$ é maximal em $\hat{S}^{1}$. Analogamente, encontramos o elemento maximal em $\hat{S}^{2}$, e a expressão (5.5) garante que $\xi_{\max }^{1} \geq \xi_{\max }^{2}$.

Sejam $\left(\xi_{t}^{1}\right)_{t \geq 0}$ e $\left(\xi_{t}^{2}\right)_{t \geq 0}$ as cadeias de Markov em $\hat{S}^{i}$ começando em $\xi_{\text {max }}^{1}$ e $\xi_{\text {max }}^{2}$, respectivamente, que seguem a construção gráfica de $U$ e $\mathcal{T}$, mas ignoram as atualizações em $x_{i}, i=1, \ldots, K-1$. Em outras palavras, a censura cancela as atualizações que tiram $\xi_{t}^{i}$ de $\hat{S}^{i}$.

As cadeias de Markov $\left(\xi_{t}^{1}\right)_{t \geq 0}$ e $\left(\xi_{t}^{2}\right)_{t \geq 0}$ são irredutíveis, pois dado $\xi \in \hat{S}^{1}$, podemos encontrar $\sigma$ tal que $\hat{\sigma}=\xi$. Assim, partindo de $\sigma$, podemos construir um caminho de transições que nos leva a $\sigma^{\prime}$ (o elemento maximal descrito em (5.5)) e que não usa nenhum dos $\tau_{x_{i}}$. Projetando esse caminho com o semi-esqueleto, encontramos um caminho de transições não censuradas de $\xi$ até $\xi_{\text {max }}^{1}$. Como $\left(\xi_{t}^{i}\right)_{t \geq 0}$ são cadeias de Markov refletidas, suas respectivas medidas de equilíbrio são $\hat{\pi}\left(\cdot \mid \bar{\xi}=\bar{\sigma}^{i}\right), i=1,2$, que são as medidas uniformes em $\hat{S}^{i}$. A ordem das condições iniciais e a preservação de ordem induzida pela construção gráfica garantem que $\forall t \geq 0, \xi_{t}^{1} \geq \xi_{t}^{2}$.

Com esse acoplamento monótono entre os dois processos, o Lema 5.10 garante a veracidade da afirmação. 
Capítulo 5. O embaralhamento de transposições adjacentes visto em tempo contínuo

Afirmação 3: Sejam $\bar{\sigma}^{1}, \bar{\sigma}^{2} \in \overline{S_{N}}$. Se $\bar{\sigma}^{1} \geq \bar{\sigma}^{2}$, então $\pi\left(\cdot \mid \bar{\sigma}=\bar{\sigma}^{1}\right) \succeq$ $\pi\left(\cdot \mid \bar{\sigma}=\bar{\sigma}^{2}\right)$.

Seja $f$ uma função crescente em $S_{N}$ e definamos $\hat{f}$ em $\hat{S}_{N}$ por

$$
\hat{f}(\xi)=\pi(f(\sigma) \mid \hat{\sigma}=\xi)
$$

Pela Afirmação $1, \hat{f}$ é uma função crescente em $\hat{S}_{N}$. Assim, pela Afirmação 2 , se $\bar{\sigma}_{1} \geq \bar{\sigma}_{2}$

$$
\begin{aligned}
\pi\left(f(\sigma) \mid \bar{\sigma}=\bar{\sigma}_{1}\right) & =\hat{\pi}\left(\hat{f}(\xi) \mid \bar{\xi}=\bar{\sigma}_{1}\right) \\
& \geq \hat{\pi}\left(\hat{f}(\xi) \mid \bar{\xi}=\bar{\sigma}_{2}\right) \\
& =\pi\left(f(\sigma) \mid \bar{\sigma}=\bar{\sigma}_{2}\right) .
\end{aligned}
$$

O resultado segue pelo Lema 5.9.

Afirmação 4: Dados $i, j \in\{1, \ldots, K\}$ e $z_{1} \geq z_{2}$ dois valores admissíveis para $\bar{\sigma}(i, j)$, temos $\pi\left(\cdot \mid \bar{\sigma}(i, j)=z_{1}\right) \succeq \pi\left(\cdot \mid \bar{\sigma}(i, j)=z_{2}\right)$.

De fato, a prova do item (i) também é válida se trocarmos a grade $\left(x_{i}, x_{j}\right)_{i, j=1}^{K-1}$ por uma grade assimétrica $\left(x_{i}, y_{j}\right)_{i, j=1}^{K-1}$, e em qualquer caso os valores de $x_{i}$ não possuem nenhum papel. Assim o item (ii) corresponde ao caso do item (i) em que $K=2$.

Afirmação 5: Se $\nu$ é uma medida de probabilidade crescente em $S_{N}$, então a densidade $\bar{\nu} / \bar{\pi}$ é uma função crescente em $\overline{S_{N}}$.

Apenas devemos integrar a função $\nu / \pi$ contra a ordenação do item (i). Com efeito, como $\nu$ é crescente, $\nu / \pi$ também é. Assim, pela dominação estocástica, se $\bar{\sigma}^{1} \geq \bar{\sigma}^{2}$, temos

$$
\begin{aligned}
(\bar{\nu} / \bar{\pi})\left(\bar{\sigma}^{1}\right) & =\pi\left(\nu / \pi \mid \bar{\sigma}=\bar{\sigma}^{1}\right) \\
& \geq \pi\left(\nu / \pi \mid \bar{\sigma}=\bar{\sigma}^{2}\right) \\
& =(\bar{\nu} / \bar{\pi})\left(\bar{\sigma}^{2}\right)
\end{aligned}
$$

Afirmação 6: Se $\nu$ é uma medida de probabilidade crescente em $S_{N}$, então $\bar{\nu}_{i, j} / \bar{\pi}_{i, j}$ é uma função crescente no conjunto de valores adimissíveis para $\bar{\sigma}(i, j)$. 
Capítulo 5. O embaralhamento de transposições adjacentes visto em tempo contínuo

Agora, devemos integrar a função $\nu / \pi$ contra a ordenação do item (ii).

\section{3}

\section{Mais algumas ferramentas}

\subsection{1}

\section{A conexão com a equação do calor}

Existe uma conexão entre o embaralhamento de transposições adjacentes e difusões. Para vermos isso, basta olharmos o movimento de apenas uma carta, que corresponde a um passeio aleatório simétrico com saltos para o vizinho mais próximo no conjunto $\{1, \ldots, N\}$. Também podemos perceber essa conexão quando olhamos para a evolução da esperança de $\tilde{\sigma}_{t}(x, y)$. Lembremos que $\tilde{\sigma}_{t}(x, y)$ só pode dar uma passo para baixo quando $\tilde{\sigma}_{t}(\cdot, y)$ apresenta um máximo local em $x$, e para cima quando apresenta um mínimo local, cada caso com taxa um. Assim,

$$
\begin{aligned}
\partial_{t} \mathbb{E}\left[\tilde{\sigma}_{t}(x, y)(t)\right] & =\mathbb{E}\left[\mathbf{1}_{\left\{\tilde{\sigma}_{t}(x, y)>\max \left(\tilde{\sigma}_{t}(x-1, y), \tilde{\sigma}_{t}(x+1, y)\right)\right\}}\right] \\
& -\mathbb{E}\left[\mathbf{1}_{\left\{\tilde{\sigma}_{t}(x, y)<\min \left(\tilde{\sigma}_{t}(x-1, y), \tilde{\sigma}_{t}(x+1, y)\right)\right\}}\right] \\
& =\mathbb{E}\left[\tilde{\sigma}_{t}(x-1, y)+\tilde{\sigma}_{t}(x+1, y)-2 \tilde{\sigma}_{t}(x, y)\right],
\end{aligned}
$$

Portanto, $f(x, y, t):=\mathbb{E}\left[\tilde{\sigma}_{t}(x, y)\right]$ é a solução da equação do calor discreta unidimensional

$$
\left\{\begin{array}{c}
\partial_{t} f=\Delta_{x} f \text { em }\{1, \ldots, N-1\} \times \mathbb{R}_{+}, \\
f(0, t)=f(N, t)=0 \\
f(x, y, 0)=\tilde{\sigma}_{0}(x, y)
\end{array}\right.
$$

onde $\Delta_{x}$ denota o Laplaciano discreto sobre a coordenada $x$, e $y$ está fixado.

Lema 5.21 Para toda $\sigma_{0} \in S_{N}$ e $t \geq 0$, temos

$$
\max _{x \in\{0, \ldots, N\}} \mathbb{E}\left[\tilde{\sigma}_{t}(x, y)\right] \leq 4 \min (y, N-y) e^{-\lambda_{N} t},
$$

onde

$$
\lambda_{N}:=2\left(1-\cos \left(\frac{\pi}{N}\right)\right)=\frac{\pi^{2}}{N^{2}}(1+o(1)) .
$$

Em particular,

$$
\max _{(x, y) \in\{0, \ldots, N\}^{2}} \mathbb{E}\left[\tilde{\sigma}_{t}(x, y)\right] \leq 2 N e^{-\lambda_{N} t} .
$$

Além disso, para $\sigma_{0}=e$, temos

$$
\mathbb{E}\left[\tilde{\sigma}_{t}(x, y)\right] \geq \frac{\min (y, N-y)}{\pi} \sin \left(\frac{\pi x}{N}\right) e^{-\lambda_{N} t} .
$$


Capítulo 5. O embaralhamento de transposições adjacentes visto em tempo contínuo

Prova. A prova que segue encontra-se em [11], Lema 4.1.

Fixemos $y \in\{0, \ldots, N\}$. Podemos calcular a solução de (5.6) através da decomposição de Fourier na base das autofunções $\left(u_{i}\right)_{i=1}^{N-1}$ de $\Delta_{x}$ que são dadas por

$$
u_{i}: x \mapsto \sqrt{\frac{2}{N}} \sin \left(\frac{x i \pi}{N}\right),
$$

cujos autovalores associados são $-\lambda_{N, i}$, onde

$$
\lambda_{N, i}:=2\left(1-\cos \left(\frac{i \pi}{N}\right)\right)
$$

Portanto,

$$
f(x, y, t)=\frac{2}{N} \sum_{i=1}^{N-1} a_{i}\left(\tilde{\sigma}_{0}(\cdot, y)\right) e^{-\lambda_{N, i} t} \sin \left(\frac{x i \pi}{N}\right),
$$

onde o coeficiente de Fourier $a_{i}$ é dado por

$$
a_{i}\left(\tilde{\sigma}_{0}(\cdot, y)\right):=\sum_{x=1}^{N-1} \tilde{\sigma}_{0}(x, y) \sin \left(\frac{x i \pi}{N}\right) .
$$

Lembremos que $\tilde{\sigma}_{0}(x, y)=\sum_{z=1}^{x} \mathbf{1}_{\left\{\sigma_{0}(z) \leq y\right\}}-\frac{x y}{N}$.

Sabemos que há apenas $y$ cartas com marcadores menores ou igual a $y$. Logo, $\sum_{z=1}^{x} \mathbf{1}_{\left\{\sigma_{0}(z) \leq y\right\}} \leq y$. Portanto,

$$
\tilde{\sigma}_{0}(x, y) \leq y
$$

Além disso,

$$
\begin{gathered}
\tilde{\sigma}_{0}(x, y) \leq x-\frac{x y}{N}=x\left(1-\frac{y}{N}\right)=\left(\frac{x}{N}\right)(N-y) \leq N-y, \\
\tilde{\sigma}_{0}(x, y) \geq-\frac{x y}{N}=-\left(\frac{x}{N}\right) y \geq-y,
\end{gathered}
$$

e como há apenas $N-y$ cartas com marcadores maiores que $y$, 
Capítulo 5. O embaralhamento de transposições adjacentes visto em tempo contínuo

$$
\begin{aligned}
\tilde{\sigma}_{0}(x, y) & =\sum_{z=1}^{x}\left(1-\mathbf{1}_{\left\{\sigma_{0}(z)>y\right\}}\right)-\frac{x y}{N} \\
& =x-\sum_{z=1}^{x} \mathbf{1}_{\left\{\sigma_{0}(z)>y\right\}}-\frac{x y}{N} \\
& \geq x-(N-y)-\frac{x y}{N}=-(N-y)+x\left(1-\frac{y}{N}\right) \\
& =-(N-y)+x\left(\frac{N-y}{N}\right) \\
& \geq-(N-y) .
\end{aligned}
$$

Assim, por (5.10), (5.11), (5.12) e (5.13), temos que

$$
\left|\tilde{\sigma}_{0}(x, y)\right| \leq \min (y, N-y), \forall x \in\{0, \ldots, N\}
$$

Logo, os coeficientes de Fourier satisfazem

$$
\left|a_{i}(\tilde{\sigma}(., y))\right| \leq \min (y, N-y) N, \forall i \in\{1, \ldots, N-1\}
$$

Ademais, como $\cos \left(\frac{i \pi}{N}\right) \leq \cos \left(\frac{\pi}{N}\right)$ para todo $i \in\{1, \ldots, N-1\}$, temos que $\lambda_{N, i} \geq i \lambda_{N}$ para todo $i \in\{1, \ldots, N-1\}$, e portanto, por (5.9),

$$
|f(x, y, t)| \leq 2 \min (y, N-y) \sum_{i=1}^{N-1} e^{-\lambda_{N} t}=\frac{2 \min (y, N-y) e^{-\lambda_{N} t}}{1-e^{-\lambda_{N} t}} .
$$

Quando $e^{-\lambda_{N} t} \leq 1 / 2$, temos (5.7).

Por outro lado, se $e^{-\lambda_{N} t} \geq 1 / 2$, como $\left|\tilde{\sigma}_{t}(x, y)\right| \leq \min (y, N-y)$, temos

$$
|f(x, t)| \leq \min (y, N-y)<2 \min (y, N-y) \leq 4 \min (y, N-y) e^{-\lambda_{N} t} .
$$

Portanto (5.7) continua valendo. 
Capítulo 5. O embaralhamento de transposições adjacentes visto em tempo contínuo

Para mostrarmos (5.8), notemos que quando

$$
\begin{aligned}
\tilde{\sigma}_{0}(x, y) & =\tilde{e}(x, y)=\sum_{z=1}^{x} \mathbf{1}_{\{z \leq y\}}-\frac{x y}{N}=\min \{x, y\}-\frac{x y}{N} \\
& =\min \left\{x-\frac{x y}{N}, y-\frac{x y}{N}\right\} \\
& =\min \left\{x\left(1-\frac{y}{N}\right), \frac{y N-x y}{N}\right\} \\
& =\min \left\{x\left(\frac{N-y}{N}\right),(N-x) \frac{y}{N}\right\} \\
& \geq \min \left(x \frac{\min (y, N-y)}{N},(N-x) \frac{\min (y, N-y)}{N}\right) \\
& =\frac{\min (y, N-y)}{\pi} \min \left(\frac{x \pi}{N}, \pi-\frac{x \pi}{N}\right) .
\end{aligned}
$$

Como $\sin u \leq \min (u, \pi-u)$ para todo $u \in[0, \pi]$, temos que

$$
\forall x \in\{1, \ldots, N-1\}, \quad \tilde{\sigma}_{0}(x, y) \geq \frac{\min (y, N-y)}{\pi} \sin \left(\frac{x \pi}{N}\right) .
$$

Agora, podemos deduzir (5.8) considerando a solução de (5.6) no tempo $t$ para ambos os lados de (5.14) através da monotonicidade da solução da equação do calor com as condições iniciais dadas.

\subsection{2}

\section{A cota superior de Wilson}

Proposição 5.22 Para todo $N$ suficientemente grande e para todo $\epsilon>0$

$$
d^{N}(t) \leq 10 N e^{-t \lambda_{N}}
$$

onde $\lambda_{N}:=2(1-\cos (\pi / N))$.

Prova. A prova encontra-se em [17], Teorema 10.

\subsection{3}

\section{Apagando os marcadores e decompondo o procedimento de mistura}

Suponhamos que trocamos os marcadores das cartas de modo que cada carta cujo marcador pertença a $\left\{x_{i-1}+1, \ldots, x_{i}\right\}, i=1, \ldots, K$ agora recebe o marcador $i$. Para visualizarmos o que está acontecendo, tomemos $K=4 \mathrm{e}$ $N=52$. Assim, estamos diferenciando as cartas apenas pelos naipes: copas, ouro, espadas e paus. $O$ baralho remarcado é descrito pelo semi-esqueleto $\hat{\sigma}$.

Intuitivamente, para que $\left(\sigma_{t}\right)_{t \geq 0}$ alcance o equilíbrio é necessário que: 
Capítulo 5. O embaralhamento de transposições adjacentes visto em tempo contínuo

(i) o semi-esqueleto $\left(\hat{\sigma}_{t}\right)_{t \geq 0}$ esteja próximo da sua distribuição de equilíbrio;

(ii) condicionando a cada semi-esqueleto, a ordem das cartas com marcadores $i$ esteja perto de estarem uniformemente distribuidas.

Nesta subseção, mostraremos essas afirmações de forma mais rigorosa.

Considere a seguinte transformação das medidas que fará com que cartas cujos marcadores pertencem a $\left\{x_{i-1}+1, \ldots, x_{i}\right\}$ sejam indistinguíveis.

Definição 5.23 Seja $\tilde{S}_{N}$ o maior subgrupo de $S_{N}$ que deixa todos os conjuntos $\left\{x_{i-1}+1, \ldots, x_{i}\right\}$ invariantes.

É fácil ver que $\tilde{S_{N}}$ é isomorfo a $\otimes_{i=1}^{K} S_{\Delta x_{i}}$.

Definição 5.24 Seja $\nu$ uma medida de probabilidade em $S_{N}$. Definimos $\tilde{\nu}$ como

$$
\tilde{\nu}(\sigma):=\frac{1}{\prod_{i=1}^{K}\left(\Delta x_{i}\right) !} \sum_{\tilde{\sigma} \in \tilde{S_{N}}} \nu(\tilde{\sigma} \circ \sigma) .
$$

Notemos que o semi-esqueleto de $\sigma$ é mantido invariante pela composição à direita por um elemento de $\tilde{S_{N}}$. Logo, temos

$$
\tilde{\nu}(\sigma):=\frac{1}{\left|\tilde{S_{N}}\right|} \hat{\nu}(\hat{\sigma})
$$

Lema 5.25 Para todas as leis de probabilidade $\nu$ em $S_{N}$, temos

$$
\|\tilde{\nu}-\pi\|_{T V}=\|\hat{\nu}-\hat{\pi}\|_{T V}
$$

Trivialmente,

$$
\|\nu-\pi\|_{T V} \leq\|\hat{\nu}-\hat{\pi}\|_{T V}+\|\nu-\tilde{\nu}\|_{T V}
$$

Prova. Primeiro, vejamos que

$$
2\|\tilde{\nu}-\pi\|_{T V}=\sum_{\sigma \in S_{N}}|\tilde{\nu}(\sigma)-\pi(\sigma)|=\sum_{\xi \in \hat{S}_{N}} \sum_{\left\{\sigma \in S_{N} \mid \hat{\sigma}=\xi\right\}}|\tilde{\nu}(\sigma)-\pi(\sigma)| .
$$

Por (5.15), $\tilde{\nu}$ é constante em $\left\{\sigma \in S_{N} \mid \hat{\sigma}=\xi\right\}$ e portanto 
Capítulo 5. O embaralhamento de transposições adjacentes visto em tempo contínuo

$$
\begin{aligned}
2\|\tilde{\nu}-\pi\|_{T V} & =\sum_{\xi \in \hat{S}_{N}}\left|\sum_{\left\{\sigma \in S_{N} \mid \hat{\sigma}=\xi\right\}} \tilde{\nu}(\sigma)-\pi(\sigma)\right| \\
& =\sum_{\xi \in \hat{S}_{N}}\left|\sum_{\left\{\sigma \in S_{N} \mid \hat{\sigma}=\xi\right\}} \nu(\sigma)-\pi(\sigma)\right| \\
& =\sum_{\xi \in \hat{S}_{N}}|\hat{\nu}(\xi)-\hat{\pi}(\xi)| \\
& =2\|\hat{\nu}-\hat{\pi}\|_{T V} .
\end{aligned}
$$

\section{4}

\section{A cota superior}

Nesta seção, mostraremos que o tempo de mistura do embaralhamento de transposições adjacentes em tempo contínuo é menor ou igual a $\frac{1}{2 \pi^{2}} N^{2} \log N$.

Para obtermos tal cota superior, necessitaremos de enunciar três proposições que necessitam da seguinte notação:

Sejam $t_{1}:=\frac{N^{2}}{2 \pi^{2}}(\delta / 3) \log N, t_{2}:=\frac{N^{2}}{2 \pi^{2}}(1+2 \delta / 3) \log N, t_{3}:=\frac{N^{2}}{2 \pi^{2}}(1+\delta) \log N$ e $K:=\lceil 1 / \delta\rceil$.

Consideraremos a dinâmica $\sigma_{t}$ começando em $\sigma_{0}=e$ e vamos aderir ao seguinte esquema censurador:

- no período $\left[0, t_{1}\right]$, as atualizações em $x_{i}, i=1, \ldots, K-1$ são canceladas;

- no período $\left(t_{1}, t_{2}\right]$, não há censura;

- no período $\left[t_{2}, t_{3}\right]$, as atualizações em $x_{i}, i=1, \ldots, K-1$ são canceladas.

Chamaremos por $\nu_{t}=P_{t}^{\mathcal{C}}$ a distribuição de $\sigma_{t}$ para a dinâmica censurada. Um dos principais ingredientes na nossa prova será o fato de que como a identidade é o elemento maximal, a distribuição inicial é uma probabilidade crescente e, pela Afirmação 2 na prova da Proposição 5.15, $\nu_{t}$ é crescente para todo $t$.

A primeira proposição diz que depois do tempo $t_{1}$, a distribuição de $\nu_{t}$ não é muito diferente de $\tilde{\nu}_{t}$.

Proposição 5.26 Para todo $\epsilon>0, \delta$ suficientemente pequeno e $N$ suficientemente grande, temos para todo $t \geq t_{1},\left\|\tilde{\nu}_{t}-\nu_{t}\right\|_{T V} \leq \frac{\epsilon}{3}$. 
Capítulo 5. O embaralhamento de transposições adjacentes visto em tempo contínuo

Prova. Para todo $t \leq t_{1}$, temos que

$$
\sigma_{t} \in S_{N} \text { e } \tilde{\nu}_{t}=\tilde{\delta}_{e}
$$

onde $\tilde{\delta}_{e}$ é a probabilidade uniforme em $\tilde{S_{N}}$ e $\delta_{e}$ é a massa de Dirac na identidade $e$.

Para cada $i=1, \ldots, K$, sejam $\nu_{t}^{i}$ a lei de $\sigma_{t}$ restrita a $\left\{x_{i-1}+1, \ldots, x_{i}\right\}$ e $\pi^{i}$ a medida de equilíbrio nas permutações de $\left\{x_{i-1}+1, \ldots, x_{i}\right\}$. Usando a Proposição 5.22 para cada dinâmica em $S_{\Delta_{x_{i}}}$ e o fato de que a distância de variação total entre medidas produto é menor que a soma das distâncias de variação total das marginais, temos

$$
\begin{aligned}
\left\|\nu_{t}-\tilde{\delta}_{e}\right\|_{T V} & \leq \sum_{i=1}^{K}\left\|\nu_{t}^{i}-\pi^{i}\right\|_{T V} \leq \sum_{i=1}^{K} 10 \Delta x_{i} e^{-t \lambda \Delta x_{i}} \\
& \leq K\left[10\left(\frac{N}{K}+1\right) \exp \left(-2 t\left(1-\cos \left(\frac{\pi}{(N / K+1)}\right)\right)\right)\right]
\end{aligned}
$$

pois $\Delta x_{i} \leq N / K+1$. Tomando $t=t_{1}$ e $N \geq 10 K$, temos

$$
\begin{aligned}
\left\|\nu_{t}-\tilde{\delta}_{e}\right\|_{T V} & \leq(10 N+10 K) \exp \left(-\frac{N^{2} \delta \log N}{3 \pi^{2}}\left(1-\cos \left(\frac{\pi}{(N / K+1)}\right)\right)\right) \\
& \leq 11 N \exp \left(-\frac{N^{2} \delta \log N}{3 \pi^{2}}\left(1-\cos \left(\frac{\pi}{(N / K+1)}\right)\right)\right) \\
& \leq 11 N \exp \left(-(10 \delta)^{-1} \log N\right) \\
& \leq \frac{\epsilon}{3}
\end{aligned}
$$

desde que $N$ seja suficientemente grande, pois

$$
\frac{N^{2} \delta}{3 \pi^{2}}\left(1-\cos \left(\frac{\pi}{(N / K+1)}\right)\right) \geq \frac{1}{10 \delta} .
$$

Notemos que mostrar (5.16) é equivalente a mostrar que

$$
\frac{10 N^{2}}{3 \pi^{2}}\left(1-\cos \left(\frac{\pi}{(N / K+1)}\right)\right) \geq \frac{1}{\delta^{2}} \text {. }
$$

De fato,

$$
\cos \left(\frac{\pi}{N / K+1}\right)=1-\frac{\pi^{2}}{2(N / K+1)^{2}}+O\left(1 / N^{4}\right) .
$$

Daí, 
Capítulo 5. O embaralhamento de transposições adjacentes visto em tempo contínuo

$$
\left(1-\cos \left(\frac{\pi}{(N / K+1)}\right)\right)=\frac{\pi^{2}}{2(N / K+1)^{2}}-O\left(1 / N^{4}\right) .
$$

Logo,

$$
\begin{aligned}
\frac{10 N^{2}}{3 \pi^{2}}\left(1-\cos \left(\frac{\pi}{(N / K+1)}\right)\right) & =\frac{10 N^{2}}{6(N / K+1)^{2}}-O\left(1 / N^{2}\right) \\
& =\frac{10 N^{2} K^{2}}{6(N+K)^{2}}-O\left(1 / N^{2}\right) \\
& =\frac{10 K^{2}}{6(K / N+1)^{2}}-O\left(1 / N^{2}\right) \\
& \geq K^{2}, \text { quando } N \text { é suficientemente grande. }
\end{aligned}
$$

Como $K \geq 1 / \delta$, temos o desejado.

Agora, mostraremos que $\left\|\nu_{t}-\tilde{\nu}_{t}\right\|_{T V}$ é decrescente. Com efeito, observemos que, da Definição 5.24, $\tilde{\nu}_{t}$ é simplesmente a lei de $\sigma_{t}$ para a dinâmica com distribuição inicial $\tilde{\delta}_{e}$. O resultado segue de um argumento padrão de acoplamento.

O resultado também é válido para $t \geq t_{1}$ pois com a censura, a dinâmica é apenas o produto de $K$ dinâmicas independentes em $S_{\Delta x_{i}}, i \in\{1, \ldots, K\}$.

A segunda proposição diz que no tempo $t_{2}$, a lei do esqueleto $\bar{\sigma}_{t}$ está próxima do equilíbrio.

Proposição 5.27 Para todo $\epsilon>0, \delta>0$ e $N$ suficientemente grande, temos

$$
\left\|\bar{\nu}_{t_{2}}-\bar{\pi}\right\|_{T V} \leq \frac{\epsilon}{3}
$$

Prova. Primeiramente, esqueçamos que $K=\left\lceil\delta^{-1}\right\rceil$, pois o que faremos vale para todo $K$ finito. Também, por simplicidade, vamos supor que $N$ é par.

Para entendermos melhor a ideia, vamos explicar no caso $K=2$. Queremos mostrar que começando da distribuição $\nu_{t_{1}}$, depois de um tempo $\frac{N^{2}}{2 \pi^{2}}(1+\delta / 3) \log N$, a altura $\tilde{\sigma}(N / 2, N / 2)=\bar{\sigma}(1,1)$, que chamaremos por $\bar{\sigma}$ desde que não haja confusão, está próxima da sua distribuição de equilíbrio.

Afirmação 1: No equilíbrio, $\bar{\sigma} \approx(\sqrt{N} / 4) \mathcal{N}$, onde $\mathcal{N}$ é uma Gaussiana padrão. 
Capítulo 5. O embaralhamento de transposições adjacentes visto em tempo contínuo

De fato, notemos que $\pi\left(\bar{\sigma}(1,1)=k-\frac{N}{4}\right)=\frac{\left(\begin{array}{c}N / 2 \\ k\end{array}\right)\left(\begin{array}{c}N / 2 \\ N / 2-k\end{array}\right)}{\left(\begin{array}{c}N \\ N / 2\end{array}\right)}$. Agora basta usarmos a fórmula de Stirling para deduzirmos um Teorema Central do Limite.

Agora, usando o Lema 5.21 com o tempo $t_{2}$, obtemos para o valor esperado de $\bar{\sigma}$ em $t_{2}$ :

$$
\begin{aligned}
\nu_{t_{2}}(\bar{\sigma}) & \leq 2 N e^{-\lambda_{N}\left(t_{2}-t_{1}\right)} \\
& =2 N \exp \left\{-\left(\frac{\pi^{2}}{N^{2}}(1+o(1))\right)\left(\frac{N^{2}}{2 \pi^{2}}(1+\delta / 3) \log N\right)\right\} \\
& =2 N e^{-\left(\frac{1}{2}+\frac{\delta}{6}\right) \log N(1+o(1))} \\
& =2 N \exp \left\{\log N^{-\left(\frac{1}{2}+\frac{\delta}{6}\right)}\right\}(1+o(1)) \\
& =(2 N) N^{-\left(\frac{1}{2}+\frac{\delta}{6}\right)}(1+o(1)) \\
& =2 N^{1 / 2-\delta / 6} \\
& \leq N^{1 / 2-\delta / 10}
\end{aligned}
$$

quando $N$ é suficientemente grande.

Portanto, o valor esperado de $\bar{\sigma}$ em $t_{2}$ é muito menor que sua flutuação no equilíbrio.

Porém, isso não é suficiente para concluirmos que $\nu_{t_{2}}$ está próxima do equilíbrio. Da Afirmação 2 na prova da Proposição 5.15, $\nu_{t_{2}}$ tem densidade crescente e pela Proposição 5.20, a densidade $\bar{\nu}_{t_{2}} / \bar{\pi}$ da distribuição de $\bar{\sigma}$ é crescente. Assim, a seguinte afirmação é suficiente para concluirmos o caso $K=2$ :

Afirmação 2: Existe uma constante $C$ tal que para qualquer $N$ e qualquer medida $\nu$ tal que $\bar{\nu} / \bar{\pi}$ é crescente, temos

$$
\|\bar{\nu}-\bar{\pi}\|_{T V} \leq \frac{C \bar{\nu}(\bar{\sigma})}{\sqrt{N}}
$$

De fato, seja $\mathcal{A}:=\{x \in\{-N / 4, N / 4+1, \ldots, N / 4\} \mid \bar{\nu}(x) \geq \bar{\pi}(x)\}$.

Pela hipótese de $\nu, \mathcal{A}$ é um conjunto crescente. Além disso, pela definição da distância de variação total, temos 
Capítulo 5. O embaralhamento de transposições adjacentes visto em tempo contínuo

$$
\|\bar{\nu}-\bar{\pi}\|_{T V}=\bar{\nu}(\mathcal{A})-\bar{\pi}(\mathcal{A})
$$

Agora, vejamos que

$$
\begin{aligned}
\bar{\nu}(\bar{\sigma}) & =\bar{\nu}(\bar{\sigma}, \mathcal{A})+\bar{\nu}\left(\bar{\sigma}, \mathcal{A}^{c}\right) \\
& =\bar{\nu}(\mathcal{A}) \bar{\nu}(\bar{\sigma} \mid \mathcal{A})+\bar{\nu}\left(\mathcal{A}^{c}\right) \bar{\nu}\left(\bar{\sigma} \mid \mathcal{A}^{c}\right) .
\end{aligned}
$$

Usando a desigualdade de correlação (Lema 5.11) para as funções $f: \bar{\sigma} \mapsto \bar{\sigma}$ e $g: \bar{\sigma} \mapsto \frac{\bar{\nu}}{\overline{\bar{T}}}(\bar{\sigma})$, que é crescente pelo item (iii) da Proposição 5.20, temos

$$
\begin{aligned}
\bar{\nu}(\mathcal{A}) \bar{\nu}(\bar{\sigma} \mid \mathcal{A}) & =\bar{\pi}(\mathcal{A}) \bar{\pi}\left(\frac{\bar{\nu}}{\bar{\pi}}(\bar{\sigma}) \bar{\sigma} \mid \mathcal{A}\right) \\
& \geq \bar{\pi}(\mathcal{A}) \bar{\pi}\left(\frac{\bar{\nu}}{\bar{\pi}}(\bar{\sigma}) \mid \mathcal{A}\right) \bar{\pi}(\bar{\sigma} \mid \mathcal{A}) \\
& =\bar{\nu}(\mathcal{A}) \bar{\pi}(\bar{\sigma} \mid \mathcal{A})
\end{aligned}
$$

Analogamente,

$$
\bar{\nu}\left(\mathcal{A}^{c}\right) \bar{\nu}\left(\bar{\sigma} \mid \mathcal{A}^{c}\right) \geq \bar{\nu}\left(\mathcal{A}^{c}\right) \bar{\pi}\left(\bar{\sigma} \mid \mathcal{A}^{c}\right) .
$$

Agora, substituindo (5.18) e (5.19) em (5.17) e subtraindo

$$
0=\bar{\pi}(\bar{\sigma})=\bar{\pi}(\mathcal{A}) \bar{\pi}(\bar{\sigma} \mid \mathcal{A})+\bar{\pi}\left(\mathcal{A}^{c}\right) \bar{\pi}\left(\bar{\sigma} \mid \mathcal{A}^{c}\right)
$$

obtemos

$$
\begin{aligned}
\bar{\nu}(\bar{\sigma}) & \geq \bar{\nu}(\mathcal{A}) \bar{\pi}(\bar{\sigma} \mid \mathcal{A})+\bar{\nu}\left(\mathcal{A}^{c}\right) \bar{\pi}\left(\bar{\sigma} \mid \mathcal{A}^{c}\right) \\
& -\bar{\pi}(\mathcal{A}) \bar{\pi}(\bar{\sigma} \mid \mathcal{A})-\bar{\pi}\left(\mathcal{A}^{c}\right) \bar{\pi}\left(\bar{\sigma} \mid \mathcal{A}^{c}\right) \\
& =[\bar{\nu}(\mathcal{A})-\bar{\pi}(\mathcal{A})] \bar{\pi}(\bar{\sigma} \mid \mathcal{A})+\bar{\nu}\left(\mathcal{A}^{c}\right)-\left[\bar{\pi}\left(\mathcal{A}^{c}\right)\right] \bar{\pi}\left(\bar{\sigma} \mid \mathcal{A}^{c}\right) \\
& =\|\bar{\nu}-\bar{\pi}\|_{T V} \bar{\pi}(\bar{\sigma} \mid \mathcal{A})-\|\bar{\nu}-\bar{\pi}\|_{T V} \bar{\pi}\left(\bar{\sigma} \mid \mathcal{A}^{c}\right) \\
& =\|\bar{\nu}-\bar{\pi}\|_{T V}\left[\bar{\pi}(\bar{\sigma} \mid \mathcal{A})-\bar{\pi}\left(\bar{\sigma} \mid \mathcal{A}^{c}\right)\right] \\
& =\|\bar{\nu}-\bar{\pi}\|_{T V}\left[\bar{\pi}\left(\bar{\sigma} \mid \bar{\sigma} \geq x_{\mathcal{A}}\right)-\bar{\pi}\left(\bar{\sigma} \mid \bar{\sigma}<x_{\mathcal{A}}\right)\right]
\end{aligned}
$$

onde $x_{\mathcal{A}}$ é um elemento de $\mathcal{A}$.

Finalmente, da escala Gaussiana, temos

$$
\bar{\pi}(\bar{\sigma} \mid \bar{\sigma}>0)=-\bar{\pi}(\bar{\sigma} \mid \bar{\sigma}<0) \geq c \sqrt{N} .
$$

Portanto, 
Capítulo 5. O embaralhamento de transposições adjacentes visto em tempo contínuo

$$
\bar{\nu}(\mathcal{A}) \geq c \sqrt{N}\|\bar{\nu}-\bar{\pi}\|_{T V}
$$

Para $K \geq 3$, a ideia é basicamente a mesma. Ponhamos $v(\bar{\sigma}):=$ $\sum_{i, j=1}^{K-1} \bar{\sigma}(i, j)$ como o volume abaixo do gráfico do esqueleto. Similarmente à prova da Afirmação 2, mostra-se que se $\nu(v(\bar{\sigma}))$ é pequena com respeito às suas flutuações no equilíbrio (que são de ordem $\sqrt{N}$ ) e $\nu$ é crescente, então $\bar{\nu}$ e $\bar{\pi}$ estão próximas uma da outra. Mais formalmente:

Afirmação 3: Seja $\nu$ uma medida de probabilidade em $S_{N}$ cuja densidade com respeito a $\pi$ é crescente. Para todo $\epsilon>0$, existe $\eta(K, \epsilon)$ tal que para $N$ suficientemente grande, temos

$$
\|\bar{\nu}-\bar{\pi}\|_{T V} \leq \frac{\epsilon}{3}
$$

sempre que $\nu(v(\bar{\sigma})) \leq \sqrt{N} \eta$.

Para entender a prova dessa afirmação, veja a prova do Lema 5.5 de [11], onde desta vez, é utilizada a desigualdade FKG para permutações.

Como no caso em que $K=2$, usando o Lema 5.21, com o tempo $t_{2}$ temos

$$
\nu_{t_{2}}[v(\bar{\sigma})] \leq 2 N(K-1)^{2} e^{-\lambda_{N}\left(t_{2}-t_{1}\right)} \leq \sqrt{N} \eta
$$

onde a última desigualdade é válida para qualquer $\eta$ fixado quando $N$ é suficientemente grande.

Assim, a Afirmação 3 é suficiente para concluir nosso resultado.

Finalmente, a terceira proposição diz que no tempo $t_{3}$, a distribuição do semi-esqueleto está próxima do equilíbrio.

Proposição 5.28 Para todo $\epsilon>0, \delta>0$ e $N$ suficientemente grande, temos

$$
\left\|\hat{\nu}_{t_{3}}-\hat{\pi}\right\|_{T V} \leq \frac{2 \epsilon}{3}
$$

Prova. No período $\left[t_{2}, t_{3}\right]$, devido à censura, os valores tomados pelos conjuntos $\sigma_{t}\left(\left\{x_{i-1}+1, \ldots, x_{i}\right\}\right), i \in\{1, \ldots, K\}$, são constantes no tempo. Neste período, as dinâmicas podem ser consideradas como um produto de $K$ embaralhamentos 
Capítulo 5. O embaralhamento de transposições adjacentes visto em tempo contínuo

independentes, e a medida de equilíbrio correspondente condicionada no ponto de partida $\sigma_{t_{2}}$ é simplesmente

$$
\pi_{\sigma_{t_{2}}}:=\pi\left(\cdot \mid \sigma\left(\left\{x_{i-1}+1, \ldots, x_{i}\right\}\right)=\sigma_{t_{2}}\left(\left\{x_{i-1}+1, \ldots, x_{i}\right\}\right), \forall i \in\{1, \ldots, K\}\right)
$$

Usando a Proposição 5.22 da mesma forma que na prova da Proposição 5.26, temos para qualquer realização de $\sigma_{t_{2}}$,

$$
\begin{aligned}
& \left\|\mathbb{P}\left(\sigma_{t_{3}} \in \cdot \mid \sigma_{t_{2}}\right)-\pi_{\sigma_{t_{2}}}\right\|_{T V} \\
& \leq K\left[10\left(\frac{N}{K}+1\right) \exp \left(-2\left(t_{3}-t_{2}\right)\left(1-\cos \left(\frac{\pi}{(N / K+1)}\right)\right)\right)\right] \\
& =K\left[10\left(\frac{N}{K}+1\right) \exp \left(-2 t_{1}\left(1-\cos \left(\frac{\pi}{(N / K+1)}\right)\right)\right)\right] \\
& \leq \frac{\epsilon}{3}
\end{aligned}
$$

desde que $N$ seja escolhido suficientemente grande.

Considerando o push-forward das medidas do semi-esqueleto e integrando no evento $\left\{\bar{\sigma}_{t_{2}}=\xi\right\}$, obtemos que para todo $\xi \in \overline{S_{N}}$,

$$
\left\|\hat{\nu}_{t_{3}}(\cdot \mid \bar{\sigma}=\xi)-\hat{\pi}(\cdot \mid \bar{\sigma}=\xi)\right\|_{T V} \leq \frac{\epsilon}{3}
$$

Observe que a distribuição de $\bar{\sigma}_{t_{3}}$ é a mesma que a de $\bar{\sigma}_{t_{2}}$. De fato, com a censura, temos $\bar{\sigma}_{t_{3}}=\bar{\sigma}_{t_{2}}$ que, pela Proposição 5.27, está próxima do equilíbrio. Mais formalmente,

$$
\begin{aligned}
2\left\|\hat{\nu}_{t_{3}}-\hat{\pi}\right\|_{T V} & =\sum_{\xi \in \overline{S_{N}}} \sum_{\left\{\hat{\sigma} \in \hat{\left.S_{N} \mid \bar{\sigma}=\xi\right\}}\right.}\left|\hat{\nu}_{t_{3}}(\hat{\sigma})-\hat{\pi}(\hat{\sigma})\right| \\
\leq & \sum_{\xi \in \overline{S_{N}}} \sum_{\left\{\hat{\sigma} \in \hat{\left.S_{N} \mid \bar{\sigma}=\xi\right\}}\right.} \bar{\nu}_{t_{3}}(\xi)\left|\hat{\nu}_{t_{3}}(\hat{\sigma} \mid \bar{\sigma}=\xi)-\hat{\pi}(\hat{\sigma} \mid \bar{\sigma}=\xi)\right| \\
& +\hat{\pi}(\hat{\sigma} \mid \bar{\sigma}=\xi)\left|\bar{\nu}_{t_{3}}(\xi)-\bar{\pi}(\xi)\right| \\
& =2\left(\left\|\bar{\nu}_{t_{3}}-\bar{\pi}\right\|_{T V}+\sum_{\xi \in \overline{S_{N}}} \bar{\nu}_{t_{3}}(\xi)\left\{\hat{\nu}_{t_{3}}(\cdot \mid \bar{\sigma}=\xi)-\hat{\pi}(\cdot \mid \bar{\sigma}=\xi)\right\}_{T V}\right) \\
& \leq 4 \epsilon / 3,
\end{aligned}
$$

sendo a última desigualdade verdadeira pela Proposição 5.27 e por (5.20). 
Capítulo 5. O embaralhamento de transposições adjacentes visto em tempo contínuo

Teorema 5.29 Para todo $\epsilon>0, \delta>0$ e $N$ suficientemente grande, temos

$$
d_{N}\left((1+\delta) \frac{N^{2}}{2 \pi^{2}} \log N\right) \leq \epsilon .
$$

Prova. Pela Proposição 5.15 e pelo Lema 5.25, temos

$$
\begin{aligned}
d_{N}\left(t_{3}\right): & =\left\|P_{t_{3}}-\pi\right\|_{T V} \leq\left\|\nu_{t_{3}}-\pi\right\|_{T V} \\
& \leq\left\|\hat{\nu}_{t_{3}}-\hat{\pi}\right\|_{T V}+\left\|\tilde{\nu}_{t_{3}}-\nu_{t_{3}}\right\|_{T V} \\
& \leq \frac{2 \epsilon}{3}+\frac{\epsilon}{3}=\epsilon .
\end{aligned}
$$

Sendo a última desigualdade verdadeira pelas Proposições 5.26 e 5.28.

\section{5}

\section{A cota inferior}

Teorema 5.30 Para o embaralhamento de transposições adjacentes em tempo contínuo, temos que $T_{m i x}^{N}(\epsilon) \geq \frac{1}{2 \pi^{2}} N^{2} \log N(1-o(1))$.

Prova.(A prova de Wilson) Veja a prova do Teorema 6 de [17]. A prova no tempo contínuo é análoga.

Veremos no capítulo 7 que há uma outra maneira de encontrarmos essa cota.

\section{6}

0 cutoff

Corolário 5.31 O embaralhamento de transposições adjacentes em tempo contínuo tem cutoff.

Prova. De fato, pelos Teoremas 5.29, 5.30, e 2.18, temos que

$$
\frac{2 \pi^{2} T_{m i x}^{N}(\epsilon)}{N^{2} \log N}=1
$$

Portanto, esta sequência de cadeias de Markov tem cutoff. 


\section{6 \\ O embaralhamento de transposições adjacentes visto em tempo discreto}

Vamos considerar a seguinte maneira de embaralhar um baralho com $N$ cartas: A cada passo, com probabilidade $1 / 2$ nós trocamos as posições de um par de cartas adjacentes escolhido uniformemente ao acaso dentre as $N-1$ possíveis escolhas, e com probabilidade $1 / 2$ não fazemos nada. Este é o mesmo embaralhamento que estudamos no capítulo anterior, porém visto em tempo discreto. Mais uma vez nos perguntamos: Quantas vezes temos que embaralhar o baralho dessa maneira até que ele esteja bem embaralhado?

A cota inferior para o tempo de mistura desta cadeia foi dada por Wilson em [17] (Veja o Teorema 6.4). Obteremos a cota superior através da cota obtida quando o embaralhamento é feito em tempo contínuo.

Sejam $\left(\sigma_{n}\right)_{n \geq 0}$ e $\left(\sigma_{t}^{\prime}\right)_{t \geq 0}$ as trajetórias das cadeias em tempo discreto e contínuo, respectivamente, e denotemos por $\mathbf{P}_{n}$ a lei de $\sigma_{n}$ e $P_{t}$ a lei de $\sigma^{\prime}$. Vamos obter uma relação entre as distribuições $\mathbf{P}_{n}$ e $P_{t}$.

Proposição 6.1 Para todo $t \geq 0$, temos

$$
P_{t}=\sum_{k=0}^{\infty} \frac{(2(N-1) t)^{k} e^{-2(N-1) t}}{k !} \boldsymbol{P}_{k} .
$$

Prova. A cadeia em tempo contínuo pode ser descrita da seguinte maneira: Consideramos um processo de Poisson $\mathcal{T}$ com taxa $2(N-1)$ que é independente, isto é, $\mathcal{T}_{0}=0$ e $\mathcal{T}_{n}-\mathcal{T}_{n-1}$ são variáveis aleatórias exponenciais i.i.d. com média $1 /[2(N-1)]$ para todo $n \geq 1$. Agora, para cada $n \geq 0$,

$$
\sigma_{t}^{\prime}=\sigma_{n}, \forall t \in\left[\mathcal{T}_{n}, \mathcal{T}_{n+1}\right)
$$

Grosso modo, estamos dizendo que para ver o embaralhamento em tempo discreto através do mesmo em tempo contínuo, basta congelarmos o tempo quando os relógios estão silenciosos. 
Capítulo 6. O embaralhamento de transposições adjacentes visto em tempo discreto

Assim,

$$
\begin{aligned}
P_{t} & =\mathbb{P}\left(\sigma^{\prime}{ }_{t} \in \cdot\right)=\sum_{k=0}^{\infty} \mathbb{P}\left(\sigma^{\prime}{ }_{t} \in \cdot, t \in\left[\mathcal{T}_{k}, \mathcal{T}_{k+1}\right)\right) \\
& =\sum_{k=0}^{\infty} \mathbb{P}\left(\sigma_{k} \in \cdot t \in\left[\mathcal{T}_{k}, \mathcal{T}_{k+1}\right)\right) \\
& =\sum_{k=0}^{\infty} \mathbb{P}\left(\sigma_{k} \in \cdot\right) \mathbb{P}\left(t \in\left[\mathcal{T}_{k}, \mathcal{T}_{k+1}\right)\right) \\
& =\sum_{k=0}^{\infty} \frac{(2(N-1) t)^{k} e^{-2(N-1) t}}{k !} \mathbf{P}_{k} .
\end{aligned}
$$

Agora, veremos um lema que nos ajudará a encontrar o tempo de mistura para o embaralhamento comparando a distância do equilíbrio deste com a do mesmo feito em tempo contínuo.

Lema 6.2 Para todos $t, n>0$, temos

$$
\left\|P_{t}-\pi\right\|_{T V} \leq \frac{\left\|\boldsymbol{P}_{n}-\pi\right\|_{T V}}{\sum_{k=0}^{n}(2(N-1) t)^{k} e^{-2(N-1) t} / k !}
$$

$e$

$$
\left\|P_{t}-\pi\right\|_{T V} \geq \frac{\left\|\boldsymbol{P}_{n}-\pi\right\|_{T V}-\sum_{k=0}^{n-1}(2(N-1) t)^{k} e^{-2(N-1) t} / k !}{\sum_{k=n}^{\infty}(2(N-1) t)^{k} e^{-2(N-1) t} / k !} .
$$

Prova. Sejam $t, n>0$. Pela Afirmação 2 na prova da Proposição 5.15 (cuja prova é análoga em tempo discreto), $\mathbf{P}_{n}$ e $P_{t}$ são medidas de probabilidade crescentes. Logo,

$$
A_{1}:=\left\{\sigma ; \mathbf{P}_{n}(\sigma) \geq \pi(\sigma)\right\}
$$

e

$$
A_{2}:=\left\{\sigma ; P_{t}(\sigma) \geq \pi(\sigma)\right\}
$$

são eventos crescentes.

Pela Afirmação 1 na prova da proposição 5.15, para qualquer evento crescente $A,\left(\mathbf{P}_{k}(A)\right)_{k \geq 0}$ é uma sequência não crescente que converge para $\pi(A)$.

Assim, 
Capítulo 6. O embaralhamento de transposições adjacentes visto em tempo discreto

$$
\begin{aligned}
\left\|P_{t}-\pi\right\|_{T V} & \geq P_{t}\left(A_{1}\right)-\pi\left(A_{1}\right) \\
& =\sum_{k=0}^{\infty}\left(\frac{(2(N-1) t)^{k} e^{-2(N-1) t}}{k !}\left(\mathbf{P}_{k}\left(A_{1}\right)-\pi\left(A_{1}\right)\right)\right) \\
& =\sum_{k=0}^{n}\left(\frac{(2(N-1) t)^{k} e^{-2(N-1) t}}{k !}\left(\mathbf{P}_{k}\left(A_{1}\right)-\pi\left(A_{1}\right)\right)\right) \\
& +\sum_{k=n+1}^{\infty}\left(\frac{(2(N-1) t)^{k} e^{-2(N-1) t}}{k !}\left(\mathbf{P}_{k}\left(A_{1}\right)-\pi\left(A_{1}\right)\right)\right) \\
& \geq \sum_{k=0}^{n}\left(\frac{(2(N-1) t)^{k} e^{-2(N-1) t}}{k !}\right)\left(\mathbf{P}_{n}\left(A_{1}\right)-\pi\left(A_{1}\right)\right) \\
& =\sum_{k=0}^{n}\left(\frac{(2(N-1) t)^{k} e^{-2(N-1) t}}{k !}\right)\left(\mathbf{P}_{n}\left(A_{1}\right)-\pi\left(A_{1}\right)\right) \\
k ! & \left.k)^{k} e^{-2(N-1) t}\left(\mathbf{P}_{k}\left(A_{1}\right)-\pi\left(A_{1}\right)\right)\right)
\end{aligned}
$$

Além disso,

$$
\begin{aligned}
& \left\|P_{t}-\pi\right\|_{T V}=P_{t}\left(A_{2}\right)-\pi\left(A_{2}\right) \\
& =\sum_{k=0}^{\infty}\left(\frac{(2(N-1) t)^{k} e^{-2(N-1) t}}{k !}\left(\mathbf{P}_{k}\left(A_{2}\right)-\pi\left(A_{2}\right)\right)\right) \\
& =\sum_{k=0}^{n-1}\left(\frac{(2(N-1) t)^{k} e^{-2(N-1) t}}{k !}\left(\mathbf{P}_{k}\left(A_{2}\right)-\pi\left(A_{2}\right)\right)\right) \\
& +\sum_{k=n}^{\infty}\left(\frac{(2(N-1) t)^{k} e^{-2(N-1) t}}{k !}\left(\mathbf{P}_{k}\left(A_{2}\right)-\pi\left(A_{2}\right)\right)\right) \\
& \leq \sum_{k=0}^{n-1}\left(\frac{(2(N-1) t)^{k} e^{-2(N-1) t}}{k !}\left(\mathbf{P}_{k}\left(A_{2}\right)-\pi\left(A_{2}\right)\right)\right) \\
& +\sum_{k=n}^{\infty}\left(\frac{(2(N-1) t)^{k} e^{-2(N-1) t}}{k !}\right)\left(\mathbf{P}_{n}\left(A_{2}\right)-\pi\left(A_{2}\right)\right) \\
& \leq \sum_{k=0}^{n-1}\left(\frac{(2(N-1) t)^{k} e^{-2(N-1) t}}{k !}\right) \\
& +\sum_{k=n}^{\infty}\left(\frac{(2(N-1) t)^{k} e^{-2(N-1) t}}{k !}\right)\left(\mathbf{P}_{n}\left(A_{2}\right)-\pi\left(A_{2}\right)\right) \\
& =\sum_{k=0}^{n-1}\left(\frac{(2(N-1) t)^{k} e^{-2(N-1) t}}{k !}\right) \\
& +\sum_{k=n}^{\infty}\left(\frac{(2(N-1) t)^{k} e^{-2(N-1) t}}{k !}\right)\left\|\mathbf{P}_{n}-\pi\right\|_{T V} .
\end{aligned}
$$


Capítulo 6. O embaralhamento de transposições adjacentes visto em tempo discreto

Portanto, temos o resultado.

\section{1}

\section{A cota superior}

Teorema 6.3 Para o embaralhamento de transposições adjacentes com $n$ cartas, temos que $t_{\text {mix }}^{(n)}(\epsilon) \leq \frac{1}{\pi^{2}} n^{3} \log n(1-o(1))$.

Prova. Dividiremos a prova em duas afirmações.

Afirmação 1: $\left\|\mathbf{P}_{n}-\pi\right\|_{T V} \leq\left\|P_{\left(n-n^{1 / 3}\right) /(2(N-1))}-\pi\right\|_{T V}+o(1)$.

De fato, usando o Lema $6.2 \mathrm{com} t=\frac{n-n^{1 / 3}}{2(N-1)}$, temos que

$$
\left\|\mathbf{P}_{n}-\pi\right\|_{T V} \leq \frac{\left\|P_{\left(n-n^{1 / 3}\right) /(2(N-1))}-\pi\right\|_{T V}}{A_{n}}
$$

onde $A_{n}=\sum_{k=0}^{n}\left(n-n^{1 / 3}\right)^{k} e^{-\left(n-n^{1 / 3}\right)} / k !$.

Portanto, apenas precisamos mostrar que $\lim _{n \rightarrow \infty} A_{n}=1$.

Com efeito,

$$
\begin{aligned}
A_{n} & =\sum_{k=0}^{n} \frac{\left(n-n^{1 / 3}\right)^{k} e^{-\left(n-n^{1 / 3}\right)}}{k !}=e^{-\left(n-n^{1 / 3}\right)} \sum_{k=0}^{n} \frac{\left(n-n^{1 / 3}\right)^{k}}{k !} \\
& =e^{-\left(n-n^{1 / 3}\right)} \sum_{k=0}^{\infty} \frac{\left(n-n^{1 / 3}\right)^{k}}{k !}-e^{-\left(n-n^{1 / 3}\right)} \sum_{k=n+1}^{\infty} \frac{\left(n-n^{1 / 3}\right)^{k}}{k !} \\
& =e^{-\left(n-n^{1 / 3}\right)} \sum_{k=0}^{\infty} \frac{\left(n-n^{1 / 3}\right)^{k}}{k !}-o(1) \\
& =e^{-\left(n-n^{1 / 3}\right)} e^{\left(n-n^{1 / 3}\right)}-o(1)=1-o(1) .
\end{aligned}
$$

Afirmação 2: Para todo $\epsilon>0$, e $N$ suficientemente grande temos

$$
\left\|\mathbf{P}_{N^{3} \log N / \pi^{2}}-\pi\right\|_{T V} \leq \epsilon
$$

De fato, tome $n=N^{3} \log N / \pi^{2}$. Assim, pela Afirmação 1, temos que

$$
\left\|\mathbf{P}_{N^{3} \log N / \pi^{2}}-\pi\right\|_{T V} \leq\left\|P_{\left(N^{2} \log N /\left(2 \pi^{2}\right)\right)(1-o(1))}-\pi\right\|_{T V}
$$

O resultado segue do Teorema 5.29. 
Capítulo 6. O embaralhamento de transposições adjacentes visto em tempo discreto

\section{2}

\section{A cota inferior}

Teorema 6.4 Para o embaralhamento de transposições adjacentes com $n$ cartas, temos que $t_{\text {mix }}^{(n)}(\epsilon) \geq \frac{1}{\pi^{2}} n^{3} \log n(1-o(1))$.

Prova. Veja a prova do Teorema 6 de [17] ou prossiga como fizemos na cota superior utilizando a outra desigualdade do Lema $6.2 \mathrm{com} t=\frac{n+n^{1 / 3}}{2(N-1)}$.

\section{3}

O cutoff

Corolário 6.5 O embaralhamento de transposições adjacentes em tempo discreto tem cutoff.

Prova. De fato, pelos Teoremas 6.3, 6.4, e 2.18, temos que

$$
\frac{\pi^{2} t_{m i x}^{(n)}(\epsilon)}{n^{3} \log n}=1
$$

Portanto, esta sequência de cadeias de Markov tem cutoff. 


\section{7 \\ Uma maravilhosa aplicação: Encontrando o tempo de mistura para o processo de exclusão simples simétrico}

Neste capítulo veremos como obter uma cota superior para o tempo de mistura do processo de exclusão simples simétrico através do embaralhamento de transposições adjacentes e como obter uma cota inferior para embaralhamento usando a exclusão.

\section{1}

\section{O processo de exclusão simples simétrico}

Consideremos um segmento com $N$ sítios e coloquemos $k \in\{1, \cdots, N-1\}$ partículas neste segmento, com no máximo uma partícula por sítio. Agora, consideremos a seguinte dinâmica: Cada partícula pula, independentemente, com taxa igual ao número de sítios vazios em sua vizinhança, para um de seus sítios vizinhos escolhido uniformemente entre eles. Equivalentemente, ela pula com taxa um em cada um dos seus vizinhos vazios. Queremos saber quanto tempo devemos esperar para alcançarmos o equilíbrio do sistema de partículas.

Mais formalmente, considere o segmento $[0, N]$ dividido em $N$ intervalos de tamanho um. Identificaremos o intervalo $[x-1, x]$ com $x \in\{1, \cdots, N\}$ e chamaremos este intervalo de sítio. Cada um destes sítios tem dois possíveis estados: ou está vazio, ou contém uma partícula.

Quando consideramos o processo de exclusão com $k$ partículas, o espaço de estados é definido por

$$
\Omega_{N, k}:=\left\{\gamma \in\{0,1\}^{N}: \sum_{x=1}^{N} \gamma(x)=k\right\}
$$

O processo de exclusão no segmento $[0, N]$ é uma cadeia de Markov em tempo contínuo em $\Omega_{N, k}$, onde cada uma das $k$ partículas pula para o vizinho à 
Capítulo 7. Uma maravilhosa aplicação: Encontrando o tempo de mistura para o processo de exclusão simples simétrico

direita e para o vizinho à esquerda com taxa um sempre que estes sítios estiverem vazios. Veja a Figura 7.1.

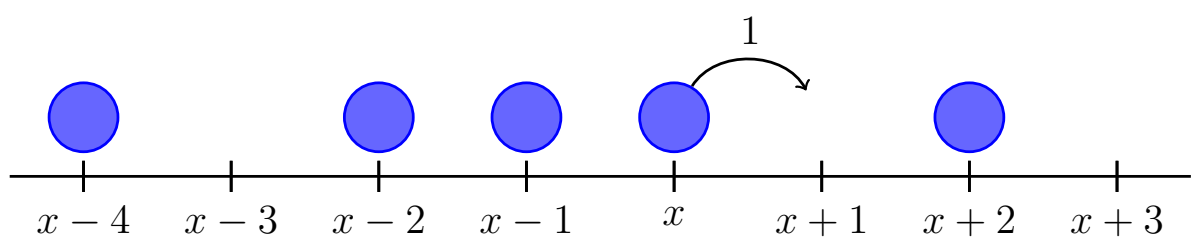

Figura 7.1: Transição de uma partícula para um sítio vizinho.

Agora, note que $S_{N}$ naturalmente atua sobre $\Omega_{N, k}$.

Para $\sigma \in S_{N}, \gamma \in \Omega_{N, k}$, podemos definir

$$
\sigma \bullet \gamma(x):=\gamma(\sigma(x))
$$

Assim, o gerador para o processo de exclusão em $[0, N]$ pode ser escrito como

$$
(\mathcal{L} f)(\gamma):=\sum_{x=1}^{N-1} f\left(\tau_{x} \bullet \gamma\right)-f(\gamma)
$$

onde $\tau_{x}$ denota a transposição adjacente $(x, x+1)$.

Chamaremos por $\pi_{k}$ (ou $\pi$ quando não houver possibilidade de confusão) a medida de equilíbrio desta cadeia. Escreveremos $\left(\gamma_{t}^{\xi}\right)_{t \geq 0}$ para a cadeia de Markov começando em $\xi \in \Omega_{N, k}$, $P_{t}^{\xi}$ para a lei da marginal $\gamma_{t}^{\xi}$ e definiremos

$$
\begin{gathered}
d^{N, k}(t):=\max _{\left\{\xi \in \Omega_{N, k}\right\}}\left\|P_{t}^{\xi}-\pi\right\|_{T V}=\max _{\left\{\nu \text { probabilidade em } \Omega_{N, k}\right\}}\left\|P_{t}^{\nu}-\pi\right\|_{T V}, \\
T_{\text {mix }}^{N, k}(\epsilon):=\inf \left\{t \geq 0: d^{N, k}(t) \leq \epsilon\right\},
\end{gathered}
$$

Note que, diferente do embaralhamento de transposições adjacentes, $\left\|P_{t}^{\xi}-\pi\right\|_{T V}$ depende da condição inicial $\xi$ pois não há simetria. 
Capítulo 7. Uma maravilhosa aplicação: Encontrando o tempo de mistura para o processo de exclusão simples simétrico

\section{Conectando a exclusão simples e o embaralhamento de transposições adjacentes}

Existe uma projeção natural do conjunto das permutações no conjunto das configurações das partículas $\sigma \mapsto \gamma_{\sigma}$. Como na Figura 7.2, à cada carta marcada de 1 até $k$, associamos o papel de uma partícula e às cartas marcadas de $k+1$ até $N$, associamos o papel de sítios vazios. Assim,

$$
\gamma_{\sigma}(x):= \begin{cases}1 & \text { se } \sigma(x) \leq k \\ 0 & \text { se } \sigma(x)>k\end{cases}
$$
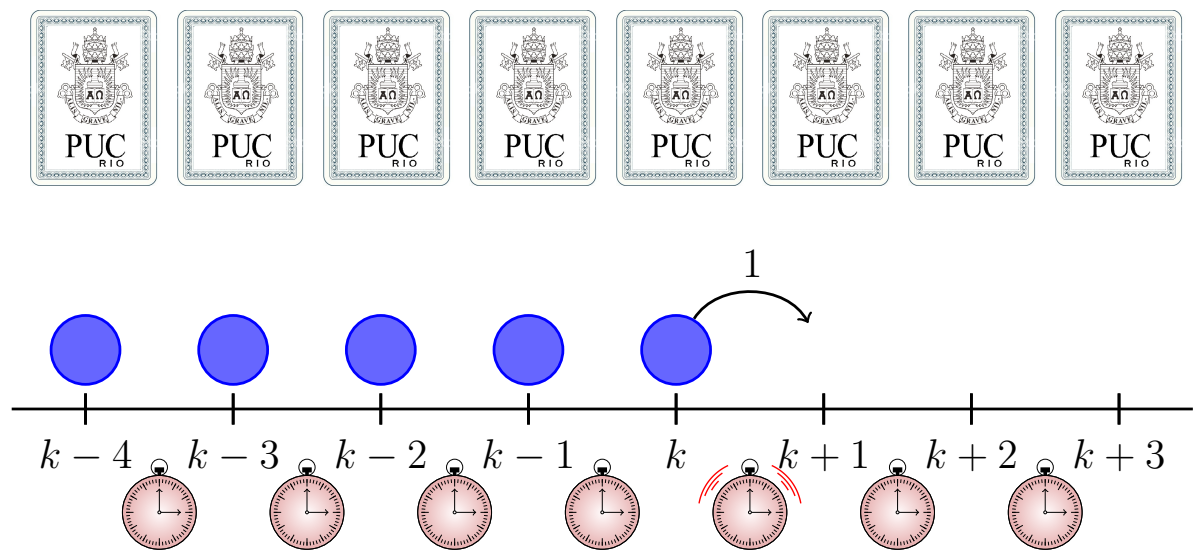

Figura 7.2: Permutação e associada à configuração inicial das partículas na construção gráfica.

Com essa aplicação, o embaralhamento de transposições adjacentes $\left(\sigma_{t}\right)_{t \geq 0}$ é projetado sobre o processo de exclusão, e como a distância só pode ser reduzida com projeção, temos que para todo $k \in\{1, \cdots, N-1\}$, para todo $t \geq 0$ e para todo $\epsilon \in(0,1)$,

$$
d^{N, k}(t) \leq d^{N}(t)
$$

$\mathrm{e}$

$$
T_{m i x}^{N, k}(\epsilon) \leq T_{m i x}^{N}(\epsilon) .
$$

Portanto, encontrando uma cota superior para o tempo de mistura do embaralhamento de transposições adjacentes, também encontramos uma cota superior para o processo de exclusão, e encontrando uma cota inferior para a 
Capítulo 7. Uma maravilhosa aplicação: Encontrando o tempo de mistura para o processo de exclusão simples simétrico

exclusão, encontramos uma cota inferior para o embaralhamento.

Nosso interesse está em utilizar a cota superior encontrada para o embaraIhamento de transposições adjacentes com o objetivo de obter uma cota superior para o sistema de partículas introduzido. Todavia, por questão de completitude, apresentaremos, sem provas, resultados que implicam na existência de cutoff para o processo de exclusão.

\section{3}

\section{A cota superior}

Proposição 7.1 Para o processo de exclusão simples simétrico temos

$$
T_{m i x}^{N, k}(\epsilon) \leq \frac{1}{2 \pi^{2}} N^{2} \log N(1+o(1)) .
$$

Prova. De fato, basta considerarmos (7.1) e o Teorema 5.29.

Em [11], Lacoin mostra uma cota ainda melhor para o tempo de mistura do processo de exclusão, que coincide com a cota obtida acima quando $k=N / 2$. Vejamos tal resultado:

Teorema 7.2 Para o processo de exclusão simples simétrico temos

$$
T_{m i x}^{N, k}(\epsilon) \leq \frac{1}{2 \pi^{2}} N^{2} \log \min (k, N-k)(1+o(1)) .
$$

Prova. Veja a seção 8 de [11].

Na demonstração desse resultado são usadas algumas das ferramentas apresentadas no capítulo 5 , mas não apenas essas.

\section{4}

\section{A cota inferior}

Teorema 7.3 Para o processo de exclusão simples simétrico temos

$$
T_{m i x}^{N, k}(\epsilon) \geq \frac{1}{2 \pi^{2}} N^{2} \log \min (k, N-k)(1-o(1)) .
$$

Prova. Veja a seção 7 de [11].

Observe que o Teorema 5.30 é imediatamente provado usando (7.1) e o Teorema 7.3 com $k=N / 2$. 
Capítulo 7. Uma maravilhosa aplicação: Encontrando o tempo de mistura para o processo de exclusão simples simétrico

7.5

O cutoff

Corolário 7.4 O processo de exclusão simples simétrico tem cutoff.

Prova. De fato, pelos Teoremas 7.2 e 7.3, temos que

$$
\frac{2 \pi^{2} T_{m i x}^{N, k}(\epsilon)}{N^{2} \log \min (k, N-k)}=1 .
$$

Portanto, esta sequência de cadeias de Markov tem cutoff. 


\section{Referências bibliográficas}

[1] ALDOUS, D., DIACONIS, P. Shuffling cards and stopping times, The American Mathematical Monthly 93.5 (1986): 333-348.

[2] ALDOUS, D., DIACONIS, P. Strong uniform times and finite random walks, Advances in Applied Mathematics 8.1 (1987): 69-97.

[3] BAYER, D., DIACONIS, P. Trailing the dovetail shuffle to its lair, The Annals of Applied Probability (1992): 294-313.

[4] BERESTYCKI, N. Lectures on Mixing Times, Cambridge University (2014).

[5] DIACONIS, P. Group representations in probability and statistics, Lecture Notes-Monograph Series 11 (1988): i-192.

[6] DURRETT, R. Probability: theory and examples, Cambridge University Press, 2010.

[7] FELLER, W. Theory of Probability and its Applications, vol. 2. (1971).

[8] HÄGGSTRÖM, O. Finite Markov chains and algorithmic applications, Vol. 52. Cambridge University Press, 2002.

[9] HOLLEY, R. Remarks on the FKG inequalities, Communications in Mathematical Physics 36.3 (1974): 227-231.

[10] JAMES, B. R. Probabilidade: Um Curso em Nível Intermediário, Coleção Euclides, Rio de Janeiro. IMPA, $3^{a}$ Edição (2011).

[11] LACOIN, H. Mixing time and cutoff for the adjacent transposition shuffle and the simple exclusion, The Annals of Probability 44.2 (2016): 1426-1487.

[12] LEVIN, D. A., PERES, Y., WILMER, E.L. Markov chains and mixing times, American Mathematical Soc., 2009.

[13] LIGGETT, T.M. Interacting particle systems, Vol. 276. Springer Science and Business Media, 2012. 
[14] LIGGETT, T. M. Continuous time Markov processes: an introduction, Vol. 113. American Mathematical Soc., 2010.

[15] PERES, Y., PETER, W. Can extra updates delay mixing?, Communications in Mathematical Physics 323.3 (2013): 1007-1016.

[16] TANNY, S. A probabilistic interpretation of Eulerian numbers, Duke Math. J 40.4 (1973): 717-722.

[17] WILSON, D. B. Mixing times of lozenge tiling and card shuffling Markov chains, Annals of Applied Probability (2004): 274-325. 
A

\section{Cadeias de Markov em espaços finitos}

\section{A.1}

\section{Cadeias de Markov em tempo discreto}

Definição A.1 Seja $P$ uma matriz $n \times n$ com elementos $P\left(w_{i}, w_{j}\right):=P_{i, j}$, $i, j=1, \ldots, n$. Chamamos de Cadeia de Markov homogênea um processo aleatório $\left(X_{0}, X_{1}, X_{2}, \ldots\right)$, com espaco de estados finito $\Omega=\left\{w_{1}, \cdots, w_{n}\right\}$, se para todo $t \geq 0$, todo $i, j \in 1, \cdots, n$ e todo $i_{0}, \cdots, i_{t-1} \in 1, \cdots, n$, temos:

$$
\begin{gathered}
P\left(X_{t+1}=w_{j} \mid X_{0}=w_{i_{0}}, \ldots, X_{t-1}=w_{i_{t-1}}, X_{t}=w_{i}\right)=P\left(X_{t+1}=w_{j} \mid X_{t}=w_{i}\right) \\
=P\left(w_{i}, w_{j}\right)
\end{gathered}
$$

Diremos que $P$ é a matriz de transição da Cadeia de Markov e $P\left(w_{i}, w_{j}\right)$ são probabilidades de transição, ou seja, $P\left(w_{i}, w_{j}\right)$ é a probabilidade condicional de chegar ao estado $w_{j}$ no tempo $t+1$ dado que no tempo $t$ estava-se no estado $w_{i}$.

Toda matriz de transição deve seguir as seguintes condições:

- $P\left(x_{i}, x_{j}\right) \geq 0, \forall i, j \in\{1, \cdots, n\}$

- $\sum_{j=1}^{n} P\left(x_{i}, x_{j}\right)=1, \forall i \in\{1, \cdots, n\}$.

Além disso, definimos as distribuções da Cadeia de Markov nos tempos $0,1,2, \cdots$ por $\mu^{(0)}, \mu^{(1)}, \mu^{(2)}, \cdots$ de forma que

$$
\begin{aligned}
\mu^{(t)} & =\left(\mu_{1}^{(t)}, \mu_{2}^{(t)}, \cdots, \mu_{n}^{(t)}\right) \\
& =\left(P\left(X_{t}=w_{1}\right), P\left(X_{t}=w_{2}\right), \ldots, P\left(X_{t}=w_{n}\right)\right) .
\end{aligned}
$$

Teorema A.2 Para uma cadeia de Markov $\left(X_{0}, X_{1}, \ldots\right)$ com espaço de estados $\Omega=\left\{w_{1}, w_{2}, \ldots, w_{n}\right\}$, distribuição inicial $\mu^{(0)}$ e matriz de transição $P$, temos que para todo $t$, a distribuição $\mu^{(t)}$ satisfaz em tempo t:

$$
\mu^{(t)}=\mu^{(0)} \cdot P^{t}
$$


Prova.Considere o caso $t=1$. Temos que para $j=1, \cdots, n$

$$
\begin{aligned}
\mu_{j}^{(1)} & =P\left(X_{1}=w_{j}\right)=\sum_{i=1}^{n} P\left(X_{0}=w_{i}, X_{1}=w_{j}\right) \\
& =\sum_{i=1}^{n} P\left(X_{0}=w_{i}\right) P\left(X_{1}=w_{j} \mid X_{0}=w_{i}\right) \\
& =\sum_{i=1}^{n} \mu^{(0)}{ }_{i} P_{i, j}=\left(\mu^{(0)} P\right)_{j},
\end{aligned}
$$

onde $\left(\mu^{(0)} \cdot P\right)_{j}$ é o $j$-ésimo elemento de $\mu^{(0)}$.P. Portanto, $\mu^{(1)}=\mu^{(0)} . P$.

Agora, suponhamos que para um $t$ fixo, $\mu^{(t)}=\mu^{(0)}$. $P^{t}$. Mostraremos que $\mu^{(t+1)}=$ $\mu^{(0)} \cdot P^{t+1}$. Com efeito,

$$
\begin{aligned}
\mu_{j}^{(t+1)} & =P\left(X_{t+1}=w_{j}\right)=\sum_{i=1}^{n} P\left(X_{t}=w_{i}, X_{t+1}=w_{j}\right) \\
& =\sum_{i=1}^{n} P\left(X_{t}=w_{i}\right) P\left(X_{t+1}=w_{j} \mid X_{t}=w_{i}\right) \\
& =\sum_{i=1}^{n} \mu_{i}^{(t)} P_{i, j}=\left(\mu^{(t)} P\right)_{j}
\end{aligned}
$$

Então,

$$
\mu^{(t+1)}=\mu^{(t)} P=\mu^{(0)} P^{t} P=\mu^{(0)} P^{t+1}
$$

Portanto, $\mu^{(t)}=\mu^{(0)} P^{t}$ para todo $t$.

Considere uma cadeia de Markov $\left(X_{0}, X_{1}, \ldots\right)$ com espaço de estados $\Omega=\left\{w_{1}, w_{2}, \ldots, w_{n}\right\}$ e matriz de transição $P$. Dizemos que um estado $w_{i}$ se comunica com outro estado $w_{j}$, com a notação $w_{i} \rightarrow w_{j}$, se a cadeia tem probabilidade positiva de alcançar $w_{j}$ saindo do estado $w_{i}$, ou seja,

$$
P\left(X_{t+s}=w_{j} \mid X_{t}=w_{i}\right)>0 \text { para algum } s \Rightarrow w_{i} \rightarrow w_{j}
$$

Se $w_{i} \rightarrow w_{j}$ e $w_{j} \rightarrow w_{i}$, então dizemos que $w_{i}$ e $w_{j}$ se intercomunicam, e usamos a notação $w_{i} \leftrightarrow w_{j}$.

Definição A.3 Dizemos que uma cadeia de Markov $\left(X_{0}, X_{1}, \ldots\right)$ com espaço de estados $\Omega=\left\{w_{1}, w_{2}, \cdots, w_{n}\right\}$ e matriz de transição $P$ é irredutível se $w_{i} \leftrightarrow w_{j}, \forall w_{i}, w_{j} \in \Omega$. Caso contrário, a cadeia é redutível.

Definição A.4 O período $d\left(w_{i}\right)$ de um estado $w_{i} \in \Omega$ é definido como

$$
d\left(w_{i}\right)=m d c\left\{t \geq 1:\left(P^{t}\right)_{i, i}>0\right\}
$$


Se $d\left(w_{i}\right)=1$, dizemos que o estado $w_{i}$ é aperiódico.

Definição A.5 Um cadeia de Markov é aperiódica se todos seus estados são aperiódicos. Caso contrário, a cadeia é periódica.

Definição A.6 Seja $\left(X_{0}, X_{1}, \ldots\right)$ uma cadeia de Markov com espaço de estados $\Omega=\left\{w_{1}, w_{2}, \cdots, w_{n}\right\}$ e matriz de transição $P$. Um vetor linha $\pi=\left(\pi_{1}, \cdots, \pi_{n}\right)$ é dito ser uma distribuição estacionária para a Cadeia de Markov, se satisfaz:

$$
\begin{aligned}
& -\pi_{i} \geq 0 \text { para } i=1, \cdots, n, \text { e } \sum_{i=1}^{n} \pi_{i}=1, \text { e } \\
& -\pi . P=\pi, \text { ou seja, } \sum_{i=1}^{n} \pi_{i} . P_{i, j}=\pi_{j} \text { para } j=1, \cdots, n .
\end{aligned}
$$

Teorema A.7 Seja $\left(X_{0}, X_{1}, \ldots\right)$ uma cadeia de Markov irredutível e aperiódica com espaço de estados $\Omega=\left\{w_{1}, w_{2}, \cdots, w_{n}\right\}$, matriz de transição $P$ e distribuição inicial $\mu^{(0)}$ arbitrária. Então, existe uma única distribuição de probabilidade $\pi$ em $\Omega$ tal que

$$
\left\|\mu^{(t)}-\pi\right\|_{T V} \rightarrow 0
$$

quando $t \rightarrow \infty$.

Prova. Veja em [8], onde é feita uma belíssima demonstração usando um acoplamento.

Definição A.8 Seja $\left(X_{0}, X_{1}, \ldots\right)$ uma cadeia de Markov com espaço de estados $\Omega=\left\{w_{1}, w_{2}, \cdots, w_{n}\right\}$ e matriz de transição $P$. Uma distribuição de probabilidade $\pi$ em $\Omega$ é reversível para a cadeia (ou para a matriz de transição $P$ ) se para todos $i, j \in\{1, \cdots, n\}$ e $t>0$ temos:

$$
\pi_{i} P_{i, j}^{t}=\pi_{j} P_{j, i}^{t}
$$

A cadeia de Markov é reversível se existe uma distribuição reversível para ela.

Teorema A.9 Seja $\left(X_{0}, X_{1}, \ldots\right)$ uma cadeia de Markov com espaço de estados $\Omega=\left\{w_{1}, w_{2}, \cdots, w_{n}\right\}$ e matriz de transição $P$. Se $\pi$ é uma distribuição reversível para a cadeia, então $\pi$ também é uma distribuição estacionária para a cadeia.

Prova. A primeira propriedade da definição de distribuição estacionária é imediata, então nos resta mostrar que para qualquer $j \in\{1, \cdots, n\}$ temos $\pi_{j}=\sum_{i=1}^{n} \pi_{i} P_{i, j}$. Com efeito,

$$
\pi_{j}=\pi_{j} \sum_{i=1}^{n} P_{j, i}=\sum_{i=1}^{n} \pi_{j} P_{j, i}=\sum_{i=1}^{n} \pi_{i} P_{i, j}
$$




\section{A.2}

\section{Cadeias de Markov em tempo contínuo}

As cadeias de Markov apresentadas nesta dissertação são exibidas com suas respectivas construções. Como o tempo agora é contínuo, falar de aperiodicidade não faria muito sentido. Assim, generalizando o que vimos, toda cadeia de Markov em tempo contínuo irredutível possui uma medida invariante $\pi$ e dada uma distribuição inicial arbitrária, a distribuição da cadeia convirgirá para $\pi$.

O gerador $\mathcal{L}$ de uma cadeia de Markov, grosso modo, explica como são feitas atualizações. Para nossa proposta, devemos saber apenas que

$$
\partial_{t} \mathbb{E}\left[f\left(X_{t}\right)\right]=\mathbb{E}\left[\mathcal{L} f\left(X_{t}\right)\right] .
$$


B

\section{Sistemas dinâmicos aleatórios}

Definição B.1 Um sistema dinâmico aleatório de uma matriz de transição $P$ num estado de espaços $\Omega$ é uma função $f: \Omega \times \Lambda \longrightarrow \Omega$, juntamente com uma variável aleatória $Z$ com valores em $\Lambda$ que satisfaz

$$
P(f(x, Z)=y)=P(x, y) .
$$

Proposição B.2 Se $Z_{1}, Z_{2}, \cdots$ é uma sequência de variáveis aleatórias independentes, cada uma tendo a mesma distribuição $Z$, e $X_{0}$ tem distribuição $\mu$, então a sequência $\left(X_{0}, X_{1}, \cdots\right)$ definida por

$$
X_{n}=f\left(X_{n-1}, Z_{n}\right), \text { para } n \geq 1
$$

é uma cadeia de Markov com matriz de transição $P$ e distribuição inicial $\mu$.

Prova. De fato,

$$
\begin{aligned}
& P\left(X_{n+1}=x_{n+1} \mid X_{n}=x_{n}, \cdots, X_{0}=x_{0}\right) \\
& =\frac{P\left(f\left(X_{n}, Z_{n+1}\right)=x_{n+1}, f\left(X_{n-1}, Z_{n}\right)=x_{n}, \cdots, f\left(X_{0}, Z_{1}\right)=x_{1}, X_{0}=x_{0}\right)}{P\left(f\left(X_{n-1}, Z_{n}\right)=x_{n}, \cdots, f\left(X_{0}, Z_{1}\right)=x_{1}, X_{0}=x_{0}\right)} \\
& =\frac{P\left(f\left(x_{n}, Z_{n+1}\right)=x_{n+1}, f\left(x_{n-1}, Z_{n}\right)=x_{n}, \cdots, f\left(x_{0}, Z_{1}\right)=x_{1}, X_{0}=x_{0}\right)}{P\left(f\left(x_{n-1}, Z_{n}\right)=x_{n}, \cdots, f\left(x_{0}, Z_{1}\right)=x_{1}, X_{0}=x_{0}\right)} \\
& =\frac{P\left(f\left(x_{n}, Z_{n+1}\right)=x_{n+1}\right) P\left(f\left(x_{n-1}, Z_{n}\right)=x_{n}\right) \cdots P\left(f\left(x_{0}, Z_{1}\right)=x_{1}\right) P\left(X_{0}=x_{0}\right)}{P\left(f\left(x_{n-1}, Z_{n}\right)=x_{n}\right) \cdots P\left(f\left(x_{0}, Z_{1}\right)=x_{1}\right) P\left(X_{0}=x_{0}\right)} \\
& =P\left(f\left(x_{n}, Z_{n+1}\right)=x_{n+1}\right)=P\left(x_{n}, x_{n+1}\right) .
\end{aligned}
$$

E por outro lado,

$$
\begin{aligned}
P\left(X_{n+1}=x_{n+1} \mid X_{n}=x_{n}\right) & =\frac{P\left(f\left(X_{n}, Z_{n+1}\right)=x_{n+1}, f\left(X_{n-1}, Z_{n}\right)=x_{n}\right)}{P\left(f\left(X_{n-1}, Z_{n}\right)=x_{n}\right)} \\
& =\frac{P\left(f\left(x_{n}, Z_{n+1}\right)=x_{n+1}, f\left(x_{n-1}, Z_{n}\right)=x_{n}\right)}{P\left(f\left(x_{n-1}, Z_{n}\right)=x_{n}\right)} \\
& =\frac{P\left(f\left(x_{n}, Z_{n+1}\right)=x_{n+1}\right) P\left(f\left(x_{n-1}, Z_{n}\right)=x_{n}\right)}{P\left(f\left(x_{n-1}, Z_{n}\right)=x_{n}\right)} \\
& =P\left(f\left(x_{n}, Z_{n+1}\right)=x_{n+1}\right)=P\left(x_{n}, x_{n+1}\right) .
\end{aligned}
$$


Proposição B.3 Toda matriz de transição em um espaço de estados finito tem um sistema dinâmico aleatório associado.

Prova. Seja $P$ a matriz de transição de uma cadeia de Markov com espaço de estados $\Omega=\left\{x_{1}, \cdots, x_{n}\right\}$. Tomemos $\Lambda=[0,1]$; Nossas variáveis aleatórias auxiliares $Z, Z_{1}, Z_{2}, \cdots$ serão uniformemente escolhidas neste intervalo.

Seja $F_{j, k}=\sum_{i=1}^{k} P\left(x_{j}, x_{i}\right)$ e definamos

$$
f\left(x_{j}, z\right):=x_{k} \text { quando } F_{j, k-1}<z \leq F_{j, k} .
$$

Assim,

$P\left(f\left(x_{j}, Z\right)=x_{k}\right)=P\left(F_{j, k-1}<Z \leq F_{j, k}\right)=P\left(x_{j}, x_{k}\right)$. 


\section{C \\ Passeios aleatórios em Grupos}

Definição C.1 Dada uma distribuição de probabilidade $Q$ em um grupo $(G,$.$) ,$ dizemos que um passeio aleatório em $G$ com distribuição de incremento $Q$ é uma cadeia de Markov (veja A.1) com espaço de estados $G$ e que se move multiplicando o estado atual, à esquerda, por um elemento aleatório de $G$ escolhido de acordo com $Q$. Equivalentemente, a matriz de transição $P$ dessa cadeia tem entradas $P(g, h g)=Q(h)$ para todo $g, h \in G$.

Partindo do princípio que $X_{0}=e$, o elemento identidade, temos que $P\left(X_{1}=g\right)=$ $Q(g)$. Além disso, a distribuição de $X_{2}$ é dada pela convolução

$$
P\left(X_{2}=g\right)=Q^{*} Q(g)=\sum_{h \in G} Q(h) Q\left(g h^{-1}\right) .
$$

Prossegindo indutivamente, $P\left(X_{k}=g\right)=Q^{k^{*}}(g)$, onde $Q^{k^{*}}$ é a convolução iterada

$$
Q^{k^{*}}=Q^{*} Q^{(k-1)^{*}}=\sum_{h \in G} Q(h) Q^{(k-1)^{*}}\left(g h^{-1}\right)
$$

Proposição C.2 Sejam $G$ um grupo finito, $P$ a matriz de transição de um passeio aleatório em $G$, e $U$ a distribuição de probabilidade uniforme em $G$. Então, $U$ é uma distribuição estacionária para $P$.

Prova. Seja $Q$ a distribuição de incremento do passeio aleatório. Para qualquer $g \in G$, temos:

$$
\sum_{h \in G} U(h) P(h, g)=\frac{1}{|G|} \sum_{k \in G} P\left(k^{-1} g, g\right)=\frac{1}{|G|} \sum_{k \in G} Q(k)=\frac{1}{|G|}=U(g) .
$$

Observação: Para a primeira igualdade, mudamos o índice fazendo $k=g h^{-1}$.

Definição C.3 Sejam $G$ um grupo, $H \subset G$ um conjunto e $\langle H\rangle$ o menor grupo contendo todos os elementos de $H$ (Lembre-se que qualquer elemento de $\langle H\rangle$ pode ser escrito como um produto de elementos em $H$ e seus inversos). Dizemos que $H$ gera $G$ se $\langle H\rangle=G$.

Proposição C.4 Sejam $G$ um grupo finito e $Q$ uma distribuição de probabilidade em $G$. O passeio aleatório em $G$ com distribuição de incremento $Q$ é irredutível se, e somente se, $S=\{g \in G: Q(g)>0\}$ gera $G$. 
Prova. Tomemos $a \in G$, arbitrariamente. Se o passeio aleatório é irredutível, então existe $r>0$ tal que $P^{r}(e, a)>0$.

Daí, deve existir uma sequência $s_{1}, \cdots, s_{r} \in G$ tal que $a=s_{r} . s_{r-1} \cdots s_{1}$ com $s_{i} \in S$ para todo $i=1, \cdots, r$. Assim, $a \in\langle S\rangle$.

Agora, suponhamos que $S$ gera $G$ e tomemos $a, b \in G$. Sabemos que $b a^{-1}$ pode ser escrito como um produto de elementos em $S$ e seus inversos. Como todo elemento de $G$ tem ordem finita, qualquer inverso aparecendo na expressão para $b a^{-1}$ pode ser reescrito como uma potência positiva do mesmo elemento do grupo.

Considere essa última expressão sendo $b a^{-1}=s_{r} . s_{r-1} \cdots s_{1}$, onde $s_{i} \in S$ para todo $i=1, \cdots, r$. Portanto,

$$
\begin{aligned}
P^{r}(a, b) & \geq P\left(a, s_{1} . a\right) P\left(s_{1} . a, s_{2} . s_{1} . a\right) \cdots P\left(s_{r-1} . s_{r-2} \cdots s_{1} . a,\left(b a^{-1}\right) a\right) \\
& =Q\left(s_{1}\right) \cdots Q\left(s_{r}\right)>0 .
\end{aligned}
$$

Definição C.5 Seja G um grupo. Dizemos que uma distribuição de probabilidade $Q$ em $G$ é simétrica se $Q(g)=Q\left(g^{-1}\right)$ para todo $g \in G$.

Proposição C.6 Seja $G$ um grupo finito. Um passeio aleatório em $G$ com distribuição de incremento $Q$ é reversível se, e somente se, $Q$ é simétrica.

Prova. Seja $U$ a distribuição de probabilidade uniforme em $G$ e suponhamos que $Q$ é simétrica. Para quaisquer $g, h \in G$ temos que

$$
U(g) P(g, h)=\frac{Q\left(h g^{-1}\right)}{|G|}=\frac{Q\left(\left(h g^{-1}\right)^{-1}\right)}{|G|}=\frac{Q\left(g h^{-1}\right)}{|G|}=U(h) P(h, g) .
$$

Reciprocamente, sejam $g, k \in G$ e tomemos $h=k g$. Assim, $k=h g^{-1}$ e como $U$ é reversível, temos:

$$
\begin{aligned}
\frac{Q(k)}{|G|} & =\frac{1}{|G|} Q\left(h g^{-1}\right)=\frac{1}{|G|} P(g, h) \\
& =\frac{1}{|G|} P(h, g)=\frac{1}{|G|} Q\left(g h^{-1}\right) \\
& =\frac{1}{|G|} Q\left(\left(h g^{-1}\right)^{-1}\right) \\
& =\frac{1}{|G|} Q\left(k^{-1}\right) .
\end{aligned}
$$


Definição C.7 Para uma distribuição $Q$ em um grupo $G$, a distribuição inversa $\hat{Q}$ é definida por $\hat{Q}(g):=Q\left(g^{-1}\right)$ para todo $g \in G$. Seja $P$ a matriz de transição de um passeio aleatório em $G$ com distribuição de incremento $Q$. Então, o passeio aleatório com distribuição de incremento $\hat{Q}$ é exatamente o tempo reverso $\hat{P}$ de $P$.

Notemos que quando $\hat{Q}=Q$, o passeio aleatório em $G$ com distribuição de incremento $Q$ é reversível e $P=\hat{P}$.

Proposição C.8 Seja $P$ a matriz de transição de um passeio aleatório em um grupo $G$ com distribuição de incremento $Q$ e seja $\hat{P}$ a matriz de transição do passeio aleatório em $G$ com distribuição de incremento $\hat{Q}$. Seja $\pi$ a distribuição uniforme em $G$. Então, para qualquer $t \geq 0$

$$
\left\|Q^{t^{*}}-\pi\right\|_{T V}=\left\|P^{t}(e, .)-\pi\right\|_{T V}=\left\|\hat{P}^{t}(e, .)-\pi\right\|_{T V}=\left\|\hat{Q}^{t^{*}}-\pi\right\|_{T V} .
$$

Prova. Seja $\left(X_{t}\right)=\left(e, X_{1}, X_{2}, \cdots\right)$ a cadeia de Markov com matriz de transição $P$ e posição inicial $e$. Podemos escrever $X_{k}$ como uma palavra $X_{k}=g_{1} g_{2} \cdots g_{k}$, onde os elementos aleatórios $g_{1}, g_{2}, \cdots \in G$ são independentes escolhas dadas pela distribuição $Q$.

Analogamente, seja $\left(Y_{t}\right)$ a cadeia de Markov com matriz de transição $\hat{P}$, com incrementos $h_{1}, h_{2}, \cdots \in G$ escolhidos independentemente sob $\hat{Q}$.

Para quaisquer elementos fixados $a_{1}, \cdots, a_{t} \in G$,

$$
\mathbb{P}\left\{g_{1}=a_{1}, \cdots, g_{t}=a_{t}\right\}=\mathbb{P}\left\{h_{1}=a_{t}^{-1}, \cdots, h_{t}=a_{1}^{-1}\right\}
$$

pela definição de $\hat{P}$.

Somando em todas as palavras tais que $a_{1} \cdots a_{t}=a$, temos

$$
P^{t}(e, a)=\hat{P}^{t}\left(e, a^{-1}\right)
$$

Portanto,

$$
\sum_{a \in G}\left|P^{t}(e, a)-\frac{1}{|G|}\right|=\sum_{a \in G}\left|\hat{P}^{t}\left(e, a^{-1}\right)-\frac{1}{|G|}\right|=\sum_{a \in G}\left|\hat{P}^{t}(e, a)-\frac{1}{|G|}\right|
$$

Logo,

$$
\left\|P^{t}(e, .)-\pi\right\|_{T V}=\left\|\hat{P}^{t}(e, .)-\pi\right\|_{T V} .
$$


D

\section{Tempos de mistura analisados}

\begin{tabular}{|l|c|}
\hline \multicolumn{1}{|c|}{ Cadeia de Markov } & Tempo de Mistura \\
\hline Embaralhamento Top-to-random & $n \log n$ \\
\hline Embaralhamento Gilbert-Shannon-Reeds & $\frac{3}{2} \log _{2} n$ \\
\hline $\begin{array}{l}\text { Embaralhamento de transposições adjacentes } \\
\text { em tempo contínuo }\end{array}$ & $\frac{1}{2 \pi^{2}} n^{2} \log n$ \\
\hline $\begin{array}{l}\text { Embaralhamento de transposições adjacentes } \\
\text { em tempo discreto }\end{array}$ & $\frac{1}{\pi^{2}} n^{3} \log n$ \\
\hline $\begin{array}{l}\text { Processo de exclusão simples simétrico } \\
\text { com k partículas }\end{array}$ & $\frac{1}{2 \pi^{2}} n^{2} \log \min (k, n-k)$ \\
\hline
\end{tabular}

Tabela D.1: Tabela com todas as cadeias de Markov analisadas e seus respectivos tempos de mistura. 